\title{
Teste do Tipo Escore para \\ Componentes de Variância em \\ Modelos Elípticos Lineares Mistos
}

Carine Savalli Redígolo

Tese apresentada

ao

Instituto de Matemática e Estatística

da

Universidade de São Paulo

para

obtenção do grau

de

Doutor em Estatística.

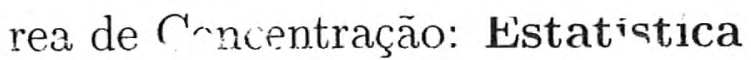

Orientador: Prof. Dr. Gilberto Alvarenga Paula

São Paulo, Janeiro de 2006 


\section{Teste do Tipo Escore para \\ Componentes de Variância em \\ Modelos Elípticos Lineares Mistos}

Este exemplar corresponde à redação final da tese devidamente corrigida e defendida por Carine Savalli Redígolo e aprovada pela banca julgadora.

São Paulo, 30 de Janeiro de 2006.

Banca examinadora:

Prof. Dr. Gilberto Alvarenga Paula (orientador) (IME-USP)

Profa. Dra. Julia Maria Pavan Soler (INE-LSP)

Prof. Dr. Francisco José de Azevedo Cysneiros (LFPE)

Profa. Dra. Clarice Garcia Borges Demetrio (ESALQ)

Prof. Dr. Filidor Edilfonso Vilca Labra (UNICAMP) 
Dedico esse trabalho ao

Fernando, ele mais do que

ninguém sabe o seu valor. 


\section{Agradecimentos}

Primeiramente ao professor Gilberto pela excelente orientação e pelo apoio, confiança e paciência que teve comigo durante todas as dificuldades e tempestades pelas quais passei para chegar até aqui.

Ao colega e grande amigo Francisco que muito me ajudou e direcionou em vários aspectos nessa tese. Ele não é oficialmente co-orientador, mas sem dúvida sem perceber assumiu esse papel.

Ao meu marido Fernando, o grande responsável por eu ter chegado até o final dessa jornada, um agradecimento muito especial, ele é o grande merecedor dessa vitória, afinal foi capaz de suportar com muito amor todas as minhas crises nesse período, e não foram poucas.... Sem ele, sem o seu constante incentivo, ombro e ajuda tudo teria sido mais difícil.

Aos meus pais Inês e Silvio e familiares que mesmo estando longe são a minha sustentação, representam a minha vontade de vencer.

Às minhas grandes amigas e companheiras de todas as horas, Paula, Lilian

e Mariana. Juntas enfrentamos muitas dificuldades e desafios no curso de doutorado. Também agradeço a todos os outros amigos que estiveram sempre por perto. O que seria de mim sem meus amigos!

Aos meus colegas de trabalhos da FIEO, especialmente Márcia e David. grandes amigos que sempre levantam meu astral.

tos professores do IME, pela oportunidade de aprofundar meus conhecimentos.

Por fim, o mais importante de todos, agradeço a Deus por me dar saúde, força e determinação, e por iluminar o meu caminho com essas pessoas que são 
verdadeiras luzes na minha vida. 


\section{Resumo}

O objetivo deste trabalho é discutir a aplicação do modelo elíptico linear misto para dados com medidas repetidas na presença de observações aberrantes. A classe das distribuições elípticas inclui várias distribuições simétricas contínuas como, por exemplo, a normal e a t-Student. Essas distribuições podem apresentar caudas mais leves do que as da normal ou mais pesadas, e este último caso apresenta-se como uma boa alternativa para tratar dados com observações aberrantes. Mostramos o processo de estimação dos parâmetros fixos e componentes de variância para o modelo elíptico linear misto, bem como para os efeitos aleatórios. Aspectos relacionados ao ajuste do modelo também são discutidos através do estudo dos resíduos condicionais padronizados e de pontos de alavanca. Maior ênfase é dada ao problema de testar hipóteses acerca dos componentes de variância da matriz de variância-covariância, já que estes se encontram na fronteira do espaço paramétrico. Para tanto, propomos utilizar a estatística do tipo escore, apresentada inicialmente por Silvapulle e Silvapulle (1995), e posteriormente, aplicada por Verbeke e Molenberghs (2003) no contexto de testes de componentes de variância para o caso normal. Finalmente, para comparar o poder da estatística do tipo escore com a estatística da razão de verossimilhanças para testar componentes de variância apresentamos os resultados de uma simulação, assumindo a distribuição t-Student. Os resultados indicam que a estatística do tipo escore, além de ser mais simples de calcular, uma vez que exige a estimação do modelo somente sob a hipótese nula, também é mais poderosa para amostras pequenas e moderadas. 


\section{Abstract}

The aim of this work is to discuss the application of the elliptical linear mixed models for repeated measures data in the presence of outlier observations. The elliptical class of distributions includes all symmetrical continuous distributions, such as, for example, normal and Student-t. These distributions can be lighter or heavier tailed than the normal distribution, and the latter presents a good choice for treating data with outliers. This work presents the estimation procedure for fixed parameters and variance components as well as for random effects in the elliptical linear mixed models. Aspects related to the model adjustment are also discussed through the study of standard conditional error and observations with high leverage. Greater emphasis is given to the problem of testing hypothesis related to the variance components, since these are in the boundary of the parameter space. In order to do so, we propose to use the score-type statistic, initially presented by Silvapulle and Silvapulle (1995), and latter applied by Verbeke and Molenberghs (2003) in the context of variance components testing for the normal case. Finally, in order to compare the power of the score-type statistic and the likelihood ratio statistic for testing variance components we present a simulation study under Student-t distribution. The results indicate that the score-type statistic, besides being simpler to calculate (as it demands the model estimation only under the null hypothesis), is also more powerful for small to moderate sample sizes. 


\section{Sumário}

1 Introdução 14

1.1 Motivação . . . . . . . . . . . . . . . . . . . . . . . . 14

1.2 Definição dos objetivos . . . . . . . . . . . . . . . . . . 19

1.3 Exemplos de aplicação . . . . . . . . . . . . . . . . . . . 21

2 Modelo elíptico linear misto $\quad 29$

2.1 Definição do modelo . . . . . . . . . . . . . . . . 29

2.2 Função escore e matriz de informação de Fisher . . . . . . . . . . 34

2.3 Estimação de máxima verossimilhança . . . . . . . . . . . . . 42

2.4 Estimação dos efeitos aleatórios . . . . . . . . . . . . . 47

2.5 Resíduo condicional . . . . . . . . . . . . . . . . . 52

2.6 Pontos de alavanca . . . . . . . . . . . . . . . . 55

2.7 Escolha da distribuição elíptica . . . . . . . . . . . . . 57

3 Teste para os componentes de variância $\quad 59$

3.1 Estatística do tipo escore . . . . . . . . . . . . . 60

3.2 Extensão para matriz $\mathbf{D}$ não-diagonal . . . . . . . . . . . . 63

3.3 Teste para um componente de variância . . . . . . . . . . . . . 64

3.4 Teste para dois componentes de variância . . . . . . . . . . † †

3.5 Teste para o componente de variância referente ao efeito aleatório na inclinação na presença do efeito aleatório no intercepto . . . . 81

4 Aplicações $\quad 85$

4.1 Dados de Potthoff-Roy . . . . . . . . . . . . . . . . 85 
4.2 Dados da altura do ramo da mandíbula . . . . . . . . . . . 89

4.3 Dados da terapia antiretroviral . . . . . . . . . . . . . . . 98

5 Estudos de simulação $\quad 112$

5.1 Teste para um efeito aleatório no intercepto . . . . . . . . . 113

$\begin{array}{lc}\text { Conclusões } & 128\end{array}$

5.2 Caso de $k=3$ restrições . . . . . . . . . . . . . . . . . . . 131

5.3 Caso de $k=4$ restrições . . . . . . . . . . . . . . . . . . 132

Referências $\quad 134$ 


\section{Lista de Figuras}

1.1 Perfis individuais da distância entre a pituitária e a fissura pterigomaxilar para os dados de Potthoff e Roy. . . . . . . . . . . . 24

1.2 Perfis individuais para a altura do ramo da mandíbula. . . . . . . 27

1.3 Perfis individuais da contagem de células CD4+ em pacientes com AIDS. . . . . . . . . . . . . . . . . . . . . . 28

2.1 Exemplos de algumas distribuições elípticas univariadas comparadas a normal (linha mais cheia). . . . . . . . . . . . .

4.1 Gráfico dos resíduos condicionais padronizados para o modelo com um efeito aleatório no intercepto assumindo distribuição normal para os dados de Potthoff-Roy. . . . . . . . . . . . . . . . . 88

4.2 Gráfico dos resíduos condicionais padronizados para o modelo com um efeito aleatório no intercepto assumindo distribuição t-Student ajustado para os dados de Potthoff-Roy.

4.3 Gráficos da alavanca generalizada para o modelo com um efeito aleatório no intercepto assumindo distribuição t-Student ajustado para os dados de Potthoff-Roy. . . . . . . . . . . . . . . . . 9

4.4 Gráfico dos pesos v's versus resíduo condicional padronizado para o modelo com um efeito aleatório no intercepto sob a suposição de distribuição t-Student para os dados de Potthoff-Roy. . . . . . . . 93

4.5 Gráfico dos pesos $v_{i}$ 's versus alavanca generalizada por observação para o modelo com um efeito aleatório no intercepto sob a suposição de distribuição t-Student para os dados de Potthoff-Roy. 
4.6 Perfis individuais ajustados para o modelo com um efeito aleatório no intercepto sob a suposição de distribuição t-Student para os dados de Potthoff-Roy. . . . . . . . . . . . . . . . . . 94

4.7 Critério da informação de Akaike para vários valores do parâmetro de forma $K$ da exponencial potência para os dados da altura do ramo da mandíbula. . . . . . . . . . . . . . . . . 96

4.8 Gráfico dos resíduos condicionais padronizados para o modelo normal com um efeito aleatório no intercepto ajustado para os dados da altura do ramo da mandíbula. . . . . . . . . . . . . . . 98

4.9 Gráficos da alavanca generalizada para o modelo final assumindo distribuição exponencial potência ajustado para os dados da altura do ramo da mandíbula. . . . . . . . . . . . . . . . . 100

4.10 Perfis individuais ajustados para o modelo com dois efeitos aleatórios no intercepto e inclinação sob a suposição de distribuição exponencial potência ajustado para os dados da altura do ramo da mandíbula. . . . . . . . . . . . . . . . . . 10

4.11 Gráfico dos pesos pelos resíduos condicionais padronizados para o modelo com dois efeitos aleatórios no intercepto e inclinação sob a suposição de distribuição exponencial potência ajustado para os dados da altura do ramo da mandíbula. . . . . . . . . . . . 102

4.12 Gráfico dos pesos pela alavanca generalizada para o modelo com dois efeitos aleatórios no intercepto e inclinação sob a suposição de distribuição exponencial potência ajustado para os dados da altura do ramo da mandíbula. . . . . . . . . . . . . . . . . . . . . 102

4.13 Critério da informação de Akaike para vários valores do número de graus de liberdade da distribuição t-Student para os dados da terapia antiretroviral. . . . . . . . . . . . . 10

4.14 Gráfico dos resíduos condicionais padronizados para o modelo normal com efeito aleatório na inclinação após a introdução da HAART ajustado para os dados da terapia antiretroviral. . . . . . . . 106 
4.15 Perfis individuais ajustados para o modelo com efeitos aleatórios na inclinação e intercepto sob a suposição de distribuição t-Student com 3 graus de liberdade ajustado para os dados da terapia antiretroviral. . . . . . . . . . . . . . . . 108

4.16 Gráficos da alavanca generalizada para o modelo com efeitos aleatórios na inclinação e intercepto sob a suposição de distribuição t-Student com 3 graus de liberdade ajustado para os dados da terapia antiretroviral. . . . . . . . . . . . . . . . 109

4.17 Gráfico dos pesos pelos resíduos condicionais padronizados para o modelo t-Student com dois efeitos aleatórios no intercepto e inclinação após a introdução da HAART ajustado para os dados da terapia antiretroviral. . . . . . . . . . . . . . . 111

4.18 Gráfico dos pesos pela alavanca generalizada para o modelo tStudent com dois efeitos aleatórios no intercepto e inclinação após a introdução da HAART ajustado para os dados da terapia antiretroviral. . . . . . . . . . . . . . . . 111

5.1 Gráficos do poder estimado das estatísticas $T_{S}$ e $R V$ para $n=20,40$ ou 60 e nível de significância de $10 \%$ para dados gerados a partir de uma distribuição t-Student multivariada com 5 graus de liberdade. 117

5.2 Gráficos do poder estimado das estatísticas $T_{S}$ e $R V$ para $n=20,40$ ou 60 e nível de significância de $5 \%$ para dados gerados a partir de uma distribuição t-Student multivariada com 5 graus de liberdade. 118

5.3 Gráficos do poder estimado das estatísticas $T_{S}$ e $R V$ para $n=20,40$ ou 60 e nível de significância de $1 \%$ para dados gerados a partir de uma distribuição t-Student multivariada com 5 graus de liberdade. 119

5.4 Gráficos do poder estimado das estatísticas $T_{S}$ e $R V$ para $n=20,40$ ou 60 e nível de significância de $10 \%$ para dados gerados a partir de uma distribuição t-Student multivariada com 10 graus de liberdade.121 
5.5 Gráficos do poder estimado das estatísticas $T_{S}$ e $R V$ para $\mathrm{n}=20,40$ ou 60 e nível de significância de $5 \%$ para dados gerados a partir de uma distribuição t-Student multivariada com 10 graus de liberdade. 122

5.6 Gráficos do poder estimado das estatísticas $T_{S}$ e $R V$ para $\mathrm{n}=20,40$ ou 60 e nível de significância de $1 \%$ para dados gerados a partir de uma distribuição t-Student multivariada com 10 graus de liberdade. 123

5.7 Gráficos do poder estimado das estatísticas $T_{S}$ e $R V$ para $\mathrm{n}=20$, 40 ou 60 e nível de significância de $10 \%$ para dados gerados a partir de uma distribuição exponencial potência multivariada com $K=0,5.125$

5.8 Gráficos do poder estimado das estatísticas $T_{S}$ e $R V$ para $\mathrm{n}=20$, 40 ou 60 e nível de significância de $5 \%$ para dados gerados a partir de uma distribuição exponencial potência multivariada com $K=0,5.126$

5.9 Gráficos do poder estimado das estatísticas $T_{S}$ e $R V$ para $\mathrm{n}=20$, 40 ou 60 e nível de significância de $1 \%$ para dados gerados a partir de uma distribuição exponencial potência multivariada com $K=0,5.127$ 


\section{Lista de Tabelas}

1.1 Distância entre a pituitária e a fissura pterigomaxilar (em milímetros) avaliada aos $8,10,12$, e 14 anos em 27 crianças. . . . . . .

1.2 Altura do ramo da mandíbula avaliada aos 8,0, 8,5, 9,0 e 9,5 anos de idade $($ ID $=$ Menino $) \ldots \ldots \ldots \ldots$

1.3 Contagem de células CD4+. O tempo (dias) 0 é o momento da introdução da terapia. Medidas feitas antes foram consideradas negativas e após foram consideradas positivas. . . . . . . . . 26

2.1 Expressões $v\left(u_{i}\right)$ para algumas distribuições elípticas. . . . . . . . 47

4.1 Resultados da estatística $\mathrm{T}_{S}$ para várias hipóteses envolvendo efeitos aleatórios sob a suposição de normalidade e distribuição tStudent em modelos lineares mistos ajustados para os dados de Potthoff-Roy. . . . . . . . . . . . . . . . .

4.2 Variação percentual nas estimativas dos parâmetros e na estatística $\mathrm{T}_{S}$ para os modelos com um efeito aleatório no intercepto sob a suposição de distribuição normal e t-Student, ajustados para os dados de Potthoff-Roy, após excluir as observações aberrantes destacadas na Figura 4.1. . . . . . . . . . . . . . . . . . . . 90

4.3 Estimativas dos parâmetros para o modelo com um efeito aleatório no intercepto sob as distribuições normal e t-Student ajustados para os dados de Potthoff-Roy. . . . . . . . . . . . . . . 90

4.4 Pesos estimados $v\left(\hat{u}_{i}\right)$ 's para o modelo t-Student com efeito aleatório no intercepto ajustado para os dados de Potthoff-Roy. . . . . 91 
4.5 Resultados da estatística $\mathrm{T}_{S}$ (p-valor) para as hipóteses consideradas sob o modelo normal e exponencial potência ajustados para os dados da altura do ramo da mandíbula. . . . . . . . . . . . 99

4.6 Estimativas dos parâmetros dos modelos normal e exponencial potência com efeitos aleatórios no intercepto e inclinação e matriz D não-diagonal ajustados para os dados da altura do ramo da mandíbula. . . . . . . . . . . . . . . . . .

4.7 Pesos estimados $v\left(\hat{u}_{i}\right)$ 's para o modelo com efeitos aleatórios no intercepto e inclinação assumindo distribuição exponencial potência para os dados da altura do ramo da mandíbula. . . . . . . . . . . 101

4.8 Resultados da estatística $\mathrm{T}_{S}$ (p-valor) para as hipóteses consideradas sob o modelo normal e t-Student com 3 graus de liberdade ajustados para os dados da terapia antiretroviral. . . . . . . . 107

4.9 Variações nas estimativas dos parâmetros e na estatística $\mathrm{T}_{S}$ do modelo com efeitos aleatórios na inclinação e intercepto sob a suposição de distribuição normal e t-Student com 3 graus de liberdade ajustado para os dados da terapia antiretroviral, após excluir as observações destacadas na Figura 4.14 . . . . . . . . . . . 107

4.10 Estimativas dos parâmetros do modelo com efeitos aleatórios na inclinação e intercepto sob a suposição de distribuição normal e t-Student com 3 graus de liberdade para os dados da terapia antiretroviral. . . . . . . . . . . . . . . . . . . . 108

4.11 Pesos estimados $v\left(\hat{u}_{i}\right)$ 's para o modelo com efeitos aleatórios na inclinação e intercepto sob a suposição de distribuição t-Student com 3 graus de liberdade para os dados da terapia antiretroviral. . 110

5.1 Valores estimados do poder para o teste de um componente de variância para dados gerados a partir de uma distribuição t-Student multivariada com 5 graus de liberdade. . . . . . . . . . . 116 
5.2 Valores estimados do poder para o teste de um componente de variância para dados gerados a partir de uma distribuição t-Student multivariada com 10 graus de liberdade. . . . . . . . . . . 120

5.3 Valores estimados do poder para o teste de um componente de variância para dados gerados a partir de uma distribuição exponencial potência multivariada com $K=0,5$. . . . . . . . . . . . 124 


\section{Capítulo 1}

\section{Introdução}

\subsection{Motivação}

A importância dos modelos mistos para analisar dados com medidas repetidas para respostas contínuas é indiscutível. Uma extensa teoria para o caso normal já está bem estabelecida na literatura, apresentada em detalhes, por exemplo, em Verbeke e Molenberghs (2001). Além disso, muitos programas computacionais estão disponíveis em vários pacotes estatísticos conhecidos.

Dados com medidas repetidas são muito freqüentes em pesquisas de diferentes áreas principalmente em Medicina e Biologia. Comumente esses estudos apresentam o objetivo de ajustar modelos simples, por exemplo polinomiais, incorporando a dependência entre as observações feitas na mesma unidade experimental. Nesse contexto, o problema consiste em estimar os parâmetros da curva de interesse e testar hipóteses acerca desses parâmetros. Muitas vezes, as variáveis são avaliadas em diferentes momentos para cada unidade experimental, e em diferentes números de vezes, caracterizando o que na literatura é denominado dados desbalanceados em relação ao tempo, para detalhes, veja por exemplo, Andreoni (1989). A grande questão em estudos com medidas repetidas é como escolher a estrutura da matriz de variâncias-covariâncias entre as observações feitas na mesma unidade experimental. Técnicas clássicas de análise multivariada podem ser adotadas para atribuir diferentes estruturas para essa matriz. Entretanto, em muitas situações o modelo misto ou modelo de efeitos aleatórios, proposto por Laird e 
Ware (1982), apresenta algumas vantagens como a de permitir tratar dados desbalanceados em relação ao tempo e modelar a matriz de variâncias-covariâncias com um número menor de parâmetros do que os modelos multivariados usuais.

O modelo de efeitos aleatórios induz uma determinada estrutura para a matriz de variâncias-covariâncias através da especificação de efeitos aleatórios no modelo estrutural e permite que cada unidade experimental tenha a sua matriz de variâncias-covariâncias própria. Uma forma alternativa interessante de interpretação desse modelo é que ele pode ser visto como um modelo hierárquico, ou em dois estágios, em que a distribuição da variável resposta consiste em um primeiro estágio e a distribuição dos efeitos aleatórios em um segundo estágio.

Para esses modelos é comum o interesse em avaliar a necessidade de inclusão de componentes de variância, de tal forma a determinar uma estrutura adequada para a matriz de variâncias-covariâncias. Nesta situação, temos que utilizar testes de hipóteses com restrições nos parâmetros, pois sob a hipótese nula os componentes de variância são colocados na fronteira do espaço paramétrico.

No processo de maximização da função de verossimilhança, as equações de estimação são geralmente obtidas a partir da expansão de Taylor, que aproxima esta função em torno de uma vizinhança, a qual deve pertencer ao espaço paramétrico. Ao maximizar a função de verossimilhança em torno de um componente de variância igual a zero, somente um lado da vizinhança pertence ao espaço paramétrico. Assim, se no processo de estimação for obtido um valor negativo para o componente de variância, este valor é truncado para zero. Ao realizar o teste da razão de verossimilhanças na fronteira do espaço paramétrico, a verossimilhança calculada sob $\mathrm{H}_{1}\left(\mathrm{~L}_{1}\right)$ pode, em virtude do truncamento do componente de variância, igualar-se à verossimilhança calculada sob $\mathrm{H}_{0}\left(\mathrm{~L}_{0}\right)$, fazendo com que a estatística da razão de verossimilhanças, dada por $-2 \log \left(\mathrm{L}_{0} / \mathrm{L}_{1}\right)$, assuma valor zero. Conseqüentemente, a distribuição desta estatística terá massa de probabilidade no ponto zero, não sendo, portanto, uma distribuição qui-quadrado com graus de liberdade dados pela diferença entre o número de parâmetros sob as hipóteses nula e alternativa, como considera a abordagem usual adotada para esta 
estatística.

Self e Liang (1987) mostraram que, no caso em que as variáveis são independentes e identicamente distribuídas, para testar a necessidade de incluir um único componente de variância, a estatística da razão de verossimilhanças deve ser comparada a uma mistura 50:50 de uma qui-quadrado com 0 grau de liberdade (um ponto com massa no valor zero) e uma qui-quadrado com 1 grau de liberdade. De uma forma mais geral, para testar a inclusão de mais um componente de variância em um modelo que já possua $q$ desses componentes, a estatística do teste deve ser comparada a uma mistura 50:50 de uma qui-quadrado com $q$ graus de liberdade e uma qui-quadrado com $(q+1)$ graus de liberdade, em que $(q+1)$ é o número de parâmetros adicionados ao modelo sob a hipótese alternativa (um componente de variância e $q$ covariâncias). Caso os efeitos aleatórios sejam considerados não correlacionados, somente um parâmetro será adicionado ao modelo sob a hipótese alternativa, e, neste caso, recaímos em uma mistura 50:50 de uma qui-quadrado com 0 grau de liberdade e uma qui-quadrado com 1 grau de liberdade.

Stram e Lee (1994) apresentaram testes para componentes de variância baseados na proposta de Self e Liang (1987), utilizando os dados de Potthoff e Roy (1964), em que foi avaliada uma medida de distância entre dois pontos da face em um grupo de 27 crianças, 11 meninas e 16 meninos. Essas crianças foram observadas aos 8, 10, 12 e 14 anos, o que caracteriza um problema de medidas repetidas. Como o objetivo era relacionar esta distância com a idade da criança, foi adotado um modelo de regressão considerando uma reta para cada criança, com efeitos aleatórios no intercepto e na inclinação.

Inicialmente, Stram e Lee (1994) consideraram um modelo mais simples apenas com efeito aleatório no intercepto. Foi testado se o componente de variância associado a este efeito aleatório deveria ser considerado. O valor da estatística da razão de verossimilhanças foi 49,6, não deixando dúvidas sobre a necessidade de incluir esse efeito. O próximo passo foi testar a necessidade de inclusão de mais um efeito aleatório, agora na inclinação. A estatística da razão de verossimilhanças obtida para este teste foi 0,833 . Pela abordagem usual, esse valor 
seria comparado a uma qui-quadrado com 1 grau de liberdade levando a um nível descritivo de $p=0,65$. Por outro lado, utilizando a mistura 50:50 de uma quiquadrado com 1 grau de liberdade e uma qui-quadrado com 2 graus de liberdade esse nível descritivo seria $p=0,51$. Esse caso evidencia que a proposta de Self e Liang proporciona testes de hipóteses menos conservadores, uma vez que para um mesmo valor da estatística o nível descritivo será menor. Portanto, em situações em que a metodologia usual resultar em níveis descritivos próximos ao nível de significância adotado, essa nova abordagem irá favorecer a rejeição da hipótese de igualdade.

Em uma avaliação mais cuidadosa, Giampaoli (1999) observou que Stram e Lee (1994) não consideraram o fato de que nos dados apresentados por Potthoff e Roy (1964) as variáveis não são independentes e identicamente distribuídas. Giampaoli (1999) também discutiu a proposta de Vu e Zhou (1997), que estenderam os resultados de Self e Liang (1987) para o caso em que as variáveis não são identicamente distribuídas e mostrou que, nos casos mais simples (modelos com até dois efeitos aleatórios), mesmo após as devidas adaptações, não houve modificação na distribuição assintótica da estatística da razão de verossimilhanças proposta por Self e Liang (1987).

Como pôde ser visto, muita ênfase tem sido dada à estatística da razão de verossimilhanças para testar os componentes de variância, entretanto, recentemente Verbeke e Molenberghs (2003) apresentaram uma alternativa à essa estatística para testar os componentes de variância, a estatística do tipo escore proposta inicialmente por Silvapulle e Silvapulle (1995). Essa estatística apresenta a grande vantagem de requerer que o modelo seja estimado somente sob a hipótese nula. Silvapulle e Silvapulle (1995) mostraram ainda que essa estatística é assintoticamente equivalente à da razão de verossimilhanças, mesmo no caso restrito.

Também importante e já bem explorada é a análise de diagnóstico para esses modelos, encontrada, por exemplo, em Hilden-Minton (1995) e Pan e Fang (2002). A análise de diagnóstico é uma ferramenta muito útil para avaliar se as 
suposições adotadas no modelo estão sendo satisfeitas, como a distribuição normal adotada para os erros, além de ser bastante útil para identificar observações aberrantes $^{1}$ ou influentes. Entretanto, existe pouca discussão na literatura sobre como proceder quando observações aberrantes são identificadas em um conjunto de dados. Particularmente, sob a suposição de normalidade as estimativas de máxima verossimilhança apresentam falta de robustez na presença dessas observações.

Conforme discutimos aqui, a teoria para o modelo de efeitos aleatórios assumindo erros normais já está bem difundida. As boas propriedades dessa distribuição somadas à sua popularidade e facilidades analíticas, a torna a grande escolha da Estatística para descrever dados contínuos. Entretanto, as inferências baseadas nessa distribuição apresentam, indiscutivelmente, essa vulnerabilidade na presença de observações aberrantes. Nesse trabalho apresentaremos uma alternativa para lidar com problemas de observações aberrantes e obter estimativas mais robustas em modelos de efeitos aleatórios. Essa proposta consiste em assumir uma distribuição simétrica com caudas mais pesadas do que as da normal para os erros com o intuito de acomodar melhor essas observações aberrantes. Essa classe de distribuições, denominada elíptica, tem sido apontada na literatura como uma boa alternativa de estimação mais robusta do que a obtida no caso normal, sendo, portanto, de grande utilidade prática.

A classe de distribuições elípticas inclui todas as distribuições simétricas contínuas, tais como a normal, t-Student, exponencial potêntia, logística I, logística II. entre outras, e suas propriedades estão apresentadas, por exemplo, em Fang, Kotz e Ng (1990). Lange. Little e Taylor (1989) discutem propriedades do modelo t-Student com estrutura longitudinal e mostram, em particular, a robustez das estimativas de máxima verossimilhança contra observações aberrantes. Pinheiro, Liu e Wu (2001) também propuseram modelos lineares mistos usando uma distribuição t-Student multivariada para os efeitos aleatórios e para os erros. Kowalski

\footnotetext{
${ }^{1}$ Definimos neste texto pontos aberrantes como sendo aqueles mal ajustados segundo o resíduo condicional padronizado.
} 
et al. (1999) apresentaram algumas propriedades assintóticas dos estimadores de máxima verossimilhança para o modelo t-Student multivariado com estrutura longitudinal. Welsch e Richardson (1997) descreveram modelos lineares mistos com distribuição t-Student multivariada para a variável resposta e Fernandez e Steel (1999) trabalharam a análise bayesiana e métodos de máxima verossimilhança com graus de liberdade desconhecidos. Mais recentemente, ainda para a distribuição t-Student multivariada, Cysneiros e Paula (2004) discutiram métodos restritos em modelos lineares com estrutura longitudinal e Taylor e Verbyla (2004) apresentaram uma modelagem conjunta dos parâmetros de locação e escala. Para outras distribuições elípticas não se encontra uma vasta literatura em dados longitudinais. Por exemplo, Lindsey (1999) discute a aplicação da distribuição exponencial potência em problemas com medidas repetidas.

Todas as questões de interesse aqui levantadas para os modelos lineares mistos que assumem distribuição normal podem ser discutidas também para outras distribuições elípticas, e, portanto, torna-se importante desenvolver métodos não só para ajustar modelos elípticos lineares mistos, mas também para estimar os efeitos aleatórios, testar componentes de variância e analisar os resíduos do modelo.

\subsection{Definição dos objetivos}

O objetivo principal deste trabalho é propor uma metodologia para testar componentes de variância em um modelo elíptico linear misto de tal forma a determinar uma estrutura para a matriz de variâncias-covariâncias adequada para os dados. Os objetivos secundários que foram desenvolvidos em consequência do objetivo principal foram:

(i) descrição da teoria de estimação dos parâmetros fixos e componentes da matriz de variâncias-covariâncias em modelos elípticos lineares mistos;

(ii) desenvolvimento de um método de estimação dos efeitos aleatórios para 
modelos elípticos lineares mistos;

(iii) desenvolvimento das expressões da estatística do tipo escore para testar os componentes de variância em vários casos;

(iv) comparação do teste do tipo escore com o teste da razão de verossimilhanças para testar a inclusão de um componente de variância.

A apresentação dos resultados obtidos neste trabalho está organizada em 5 capítulos, como descritos a seguir, e a conclusão.

No capítulo 2, o modelo elíptico linear misto será definido e suas diferenças básicas com relação ao modelo normal serão discutidas, destacando algumas propriedades das distribuições elípticas. O processo de estimação dos parâmetros fixos e dos componentes da matriz de variâncias-covariâncias será apresentado. Também a estimação dos efeitos aleatórios será tratada neste capítulo, e mostraremos que a obtenção das expressões para a variância das estimativas dos efeitos aleatórios torna-se muito mais trabalhosa no caso elíptico. Maior ênfase será dada às distribuições t-Student e exponencial potência multivariadas, uma vez que nosso principal objetivo é utilizar distribuições com caudas mais pesadas do que as da normal para acomodar valores aberrantes, característica satisfeita por ambas.

O capítulo 3 é dedicado à obtenção das expressões da estatística do tipo escore para testar os componentes de variância no modelo elíptico linear misto. Essa estatística foi primeiramente apresentada por Silvapulle e Silvapulle (1995) em um contexto geral de testes com restrições nos parâmetros, e, recentemente, utilizada por Verbeke e Molenberghs (2003) para atender ao objetivo específico de testar componentes de variância, restritos a valores não-negativos, no caso normal.

No capítulo 4, mostraremos várias aplicações de modelos de efeitos aleatórios para dados com medidas repetidas, balanceados e não-balanceados, assumindo alguma distribuição elíptica com caudas mais pesadas do que as da normal para os erros. Para todos os exemplos explorados será feito um paralelo entre os resultados obtidos com a distribuição normal e a outra distribuição elíptica adotada, destacando as vantagens do modelo elíptico. 
No capítulo 5 apresentaremos um estudo de simulação para comparar a estatística do tipo escore, proposta no capítulo 3, e a estatística da razão de verossimilhanças para o caso de testar a inclusão de um efeito aleatório no intercepto. Neste estudo de simulação o objetivo principal é comparar o poder dos dois testes para amostras pequenas e moderadas. Para cada amostra gerada serão calculados os valores das duas estatísticas e o poder dos testes será estimado como sendo a proporção de p-valores maiores do que o nível de significância adotado.

Por fim, o último capítulo traz as conclusões deste trabalho e possíveis estudos futuros relacionados.

\subsection{Exemplos de aplicação}

Os exemplos de aplicação que serão trabalhados estão descritos a seguir.

Exemplo 1.1: O conjunto de dados de ortodontia apresentado por Potthoff e Roy (1964), já bem explorado na literatura, apresenta a variável resposta distância entre a pituitária e a fissura pterigomaxilar (em milímetros), que foi medida aos 8, 10, 12, e 14 anos de idade em 27 crianças divididas em dois grupos, meninos e meninas (Tabela 1.1). A análise descritiva, apresentada na Figura 1.1, mostra que nos dois grupos a resposta parece crescer linearmente com a idade e existe uma variação no intercepto dos perfis individuais. Como aparecem alguns perfis atípicos para o grupo de meninos vamos assumir inicialmente o modelo normal tradicional e comparar os resultados supondo posteriormente erros elípticos com caudas mais pesadas para o grupo de meninos.

Exemplo 1.2: Um outro estudo de ortodontia analisado por Pan e Fang (2002, Exemplo 2.7) tentou estabelecer um padrão de crescimento para a altura do ramo da mandíbula (em milímetros). Para tanto, 20 garotos foram avaliados aos 8, 8,5, 9 e 9.5 anos de idade (Tabela 1.2). A análise descritiva, apresentada na Figura 1.2 , mostra que existe uma variação no intercepto da curva de cada indivíduo, mas 
não está evidente se existe uma variação importante na inclinação das curvas. $\mathrm{O}$ modelo normal linear misto será então aplicado e os seus resultados comparados com um modelo elíptico linear misto.

Exemplo 1.3: Para avaliar o efeito da terapia antiretroviral (HAART) no citomegalovirus (CMV), pacientes aidéticos com níveis baixos de células CD4+ foram estudados. Os pacientes foram acompanhados por mais de três meses de acordo com 3 perfis: grupo A, composto por 12 pacientes que foram acompanhados antes da introdução da terapia (HAART), grupo B, composto por 17 pacientes seguidos antes e depois da introdução da terapia e, finalmente, grupo C, composto por 10 pacientes que foram acompanhados somente após a introdução da terapia. A divisão em grupos foi feita somente para organizar a análise descritiva dos dados. Como cada paciente foi avaliado em um momento diferente e em diferentes números de vezes (1, 2 ou 3 vezes), esses dados são desbalanceados em relação ao tempo (Tabela 1.3). A análise descritiva dos perfis individuais, apresentada na Figura 1.3, indica que a contagem de células CD4+ se altera muito pouco antes da introdução da terapia. Entretanto, após esse momento, essa variável parece apresentar um crescimento importante. Um modelo de retas segmentadas com efeitos aleatórios assumindo distribuição normal para os erros foi utilizado em Borges (2001). Como a variação dos comportamentos é grande, iremos propor analisar esses dados assumindo uma distribuição com caudas mais pesadas do que as da normal para avaliar o impacto nos resultados. Observe que métodos usuais de análise multivariada não seriam adequados a esse exemplo, devido à estrutura totalmente desbalanceada presente nos dados, portanto, neste caso. o modelo com efeitos aleatórios é o mais indicado para incorporar a dependência entre as observações feitas no mesmo paciente. 
Tabela 1.1: Distância entre a pituitária e a fissura pterigomaxilar (em milímetros) avaliada aos 8, 10, 12, e 14 anos em 27 crianças.

\begin{tabular}{lcrrrr}
\hline \hline & & & Idade & (anos) & \\
\hline Grupo & Indivíduo & 8 & 10 & 12 & 14 \\
\hline Meninas & 1 & 21,0 & 20,0 & 21,5 & 23,0 \\
& 2 & 21,0 & 21,5 & 24,0 & 25,5 \\
& 3 & 20,5 & 24,0 & 24,5 & 26,0 \\
& 4 & 23,5 & 24,5 & 25,0 & 26,5 \\
& 5 & 21,5 & 23,0 & 22,5 & 23,5 \\
& 6 & 20,0 & 21,0 & 21,0 & 22,5 \\
& 7 & 21,5 & 22,5 & 23,0 & 25,0 \\
& 8 & 23,0 & 23,0 & 23,5 & 24,0 \\
& 9 & 20,0 & 21,0 & 22,0 & 21,5 \\
& 10 & 16,5 & 19,0 & 19,0 & 19,5 \\
& 11 & 24,5 & 25,0 & 28,0 & 28,0 \\
\hline 12 & 26,0 & 25,0 & 29,0 & 31,0 \\
& 13 & 21,5 & 22,5 & 23,0 & 26,5 \\
& 14 & 23,0 & 22,5 & 24,0 & 27,5 \\
& 15 & 25,5 & 27,5 & 26,5 & 27,0 \\
& 16 & 20,0 & 23,5 & 22,5 & 26,0 \\
& 17 & 24,5 & 25,5 & 27,0 & 28,5 \\
& 18 & 22,0 & 22,0 & 24,5 & 26,5 \\
& 19 & 24,0 & 21,5 & 24,5 & 25,5 \\
& 20 & 23,0 & 20,5 & 31,0 & 26,0 \\
21 & 27,5 & 28,0 & 31,0 & 31,5 \\
22 & 23,0 & 23,0 & 23,5 & 25,0 \\
& 23 & 21,5 & 23,5 & 24,0 & 28,0 \\
& 24 & 17,0 & 24,5 & 26,0 & 29,5 \\
& 25 & 22,5 & 25,5 & 25,5 & 26,0 \\
& 26 & 23,0 & 24,5 & 26,0 & 30,0 \\
& 27 & 22.0 & 21,5 & 23,5 & 25,0 \\
\hline \hline
\end{tabular}





Figura 1.1: Perfis individuais da distância entre a pituitária e a fissura pterigomaxilar para os dados de Potthoff e Roy. 
Tabela 1.2: Altura do ramo da mandíbula avaliada aos 8,0, 8,5, 9,0 e 9,5 anos de idade (ID = Menino).

\begin{tabular}{|c|c|c|c|c|c|}
\hline ID & Idade & Altura & ID & Idade & Altura \\
\hline \multirow[t]{4}{*}{1} & 8,0 & 47,8 & 11 & 8,0 & 51,2 \\
\hline & 8,5 & 48,8 & & 8,5 & 51,4 \\
\hline & 9,0 & 49,0 & & 9,0 & 51,6 \\
\hline & 9,5 & 49,7 & & 9,5 & 51,9 \\
\hline \multirow[t]{4}{*}{2} & 8,0 & 46,4 & 12 & 8,0 & 48,5 \\
\hline & 8,5 & 47,3 & & 8,5 & 49,2 \\
\hline & 9,0 & 47,7 & & 9,0 & 53,0 \\
\hline & 9,5 & 48,4 & & 9,5 & 55,6 \\
\hline \multirow[t]{4}{*}{3} & 8,0 & 46,3 & 13 & 8,0 & 52,1 \\
\hline & 8,5 & 46,8 & & 8,5 & 52,8 \\
\hline & 9,0 & 47,8 & & 9,0 & 53,7 \\
\hline & 9,5 & 48,5 & & 9,5 & 55,0 \\
\hline \multirow[t]{4}{*}{4} & 8,0 & 45,1 & 14 & 8,0 & 48,2 \\
\hline & 8,5 & 45,3 & & 8,5 & 48,9 \\
\hline & 9,0 & 46,1 & & 9,0 & 49,3 \\
\hline & 9,5 & 47,2 & & 9,5 & 49,8 \\
\hline \multirow[t]{4}{*}{5} & 8,0 & 47,6 & 15 & 8,0 & 49,6 \\
\hline & 8,5 & 48,5 & & 8,5 & 50,4 \\
\hline & 9,0 & 48,9 & & 9,0 & 51,2 \\
\hline & 9,5 & 49,3 & & 9,5 & 51,8 \\
\hline \multirow[t]{4}{*}{6} & 8,0 & 52,6 & 16 & 8,0 & 50,7 \\
\hline & 8,5 & 53,2 & & 8,5 & 51,7 \\
\hline & 9,0 & 53,3 & & 9,0 & 52,7 \\
\hline & 9,5 & 53,7 & & 9,5 & 53,3 \\
\hline \multirow[t]{4}{*}{7} & 8,0 & 51,2 & 17 & 8,0 & 47,2 \\
\hline & 8,5 & 53,0 & & 8,5 & 47,7 \\
\hline & 9,0 & 54,3 & & 9,0 & 48,4 \\
\hline & 9,5 & 54,5 & & 9,5 & 49,5 \\
\hline \multirow[t]{4}{*}{8} & 8,0 & 49,8 & 18 & 8,0 & 53,3 \\
\hline & 8,5 & 50,0 & & 8,5 & 54,6 \\
\hline & 9,0 & 50,3 & & 9,0 & 55,1 \\
\hline & 9,5 & 52,7 & & 9,5 & 55,3 \\
\hline \multirow[t]{4}{*}{9} & 8,0 & 48,1 & 19 & 8,0 & 46,2 \\
\hline & $8, \bar{j}$ & 50,8 & & 8,5 & 47,5 \\
\hline & 9,0 & 52,3 & & 9,0 & 48,1 \\
\hline & 9,5 & 54,4 & & 9,5 & 48,4 \\
\hline \multirow[t]{4}{*}{10} & 8,0 & 45,0 & 20 & 8,0 & 46,3 \\
\hline & 8,5 & 47,0 & & 8,5 & 47,6 \\
\hline & 9,0 & 47,3 & & 9,0 & 51,3 \\
\hline & 9,5 & 48.3 & & $9, \overline{5}$ & 51.8 \\
\hline
\end{tabular}


Tabela 1.3: Contagem de células CD4+. O tempo (dias) 0 é o momento da introdução da terapia. Medidas feitas antes foram consideradas negativas e após foram consideradas positivas.

\begin{tabular}{|c|c|c|c|c|c|c|c|c|}
\hline acie & $\overline{\text { Tempo }}$ & $|\overline{C D} 4|$ & Paciente & Tempo & (CD4 & Paciente & Tempo & $\overline{\mathrm{CD} 4}$ \\
\hline$\overline{\mathrm{PA}}$ & $-112,02$ & 4 & $\overline{P A C}$ & 0.00 & 24 & AC30.1 & 263,00 & 46 \\
\hline & & 7 & & & 33 & & & 60 \\
\hline & & 1 & & 0 & 9 & & & 175 \\
\hline & & 42 & & & 14 & & $-176,86$ & 18 \\
\hline & & & & & 46 & & & 18 \\
\hline & -350 & 9 & & & 149 & 3 & 299,23 & 115 \\
\hline & & 4 & & & 13 & & $-189,03$ & \\
\hline & -36 & 26 & & 280 & 67 & & 0,00 & 16 \\
\hline & & 20 & & & 18 & & 136,07 & 6 \\
\hline & $-36 c$ & 47 & & 276 & 169 & & $-196,03$ & \\
\hline & & & & $-225,86$ & 26 & & 0 & 103 \\
\hline & 329 & 20 & & 0,00 & 11 & & & 234 \\
\hline & & 4 & & & 285 & & $-182,03$ & 17 \\
\hline & -126 & 16 & & -378 & 27 & & 0.0 & 41 \\
\hline & & 5 & & & 7 & & 287,05 & 178 \\
\hline & & 41 & & 455 & 192 & & $-147,03$ & 14 \\
\hline & & 63 & & -32 & 11 & & 0,0 & 28 \\
\hline & & & & & & & 322,06 & 26 \\
\hline & & 5 & & & 14 & & $-52,05$ & 2 \\
\hline & & 24 & & -434 & 38 & & 0,0 & 16 \\
\hline & & 48 & & & 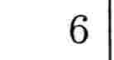 & & 415,81 & 122 \\
\hline & & 35 & & & 96 & & $-132,11$ & 11 \\
\hline & 570, & 208 & & $-350,06$ & & & 0,00 & 55 \\
\hline & & 17 & & & 109 & & 302,88 & 187 \\
\hline & & 165 & & & 130 & & $-140,94$ & \\
\hline & & & & -140 & 35 & & 0,0 & 95 \\
\hline & & 106 & & & 35 & & 266,05 & 24 \\
\hline & & 20 & & & 65 & & $-48,10$ & 39 \\
\hline & & 47 & & & 24 & & 0,00 & 65 \\
\hline & & 13 & & & 25 & PAC 39.3 & 310,79 & 1.2 \\
\hline & 530.26 & 320 & PAC'29.3 & 301,96 & 142 & & & \\
\hline
\end{tabular}




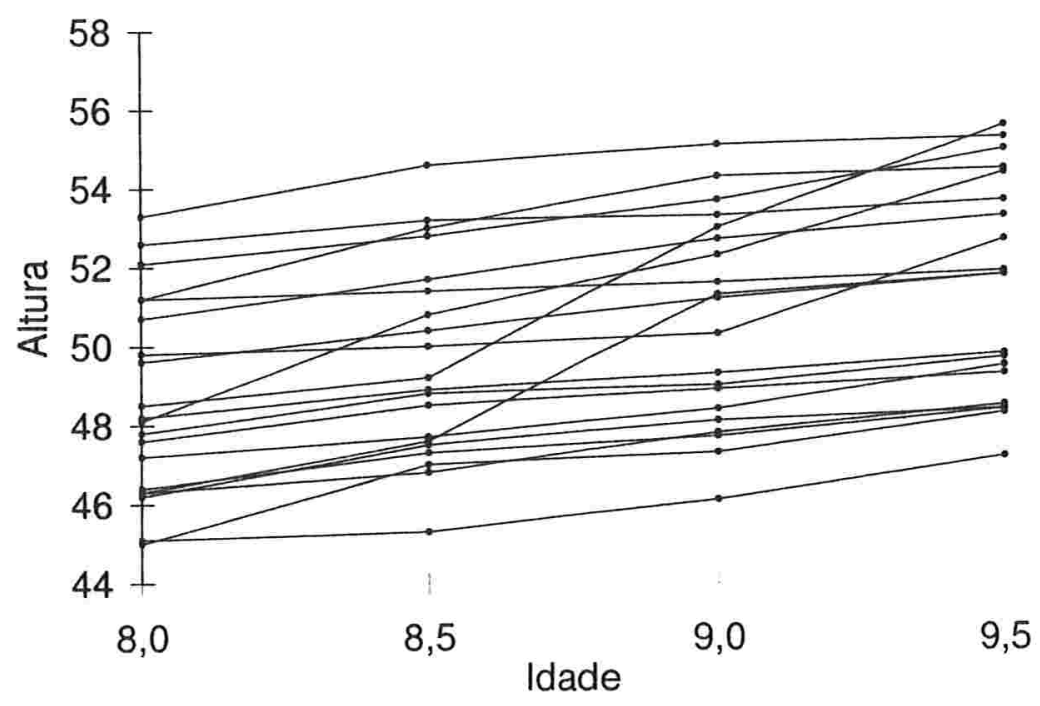

Figura 1.2: Perfis individuais para a altura do ramo da mandíbula. 




\section{Contagem de células CD4+ - Grupo B}

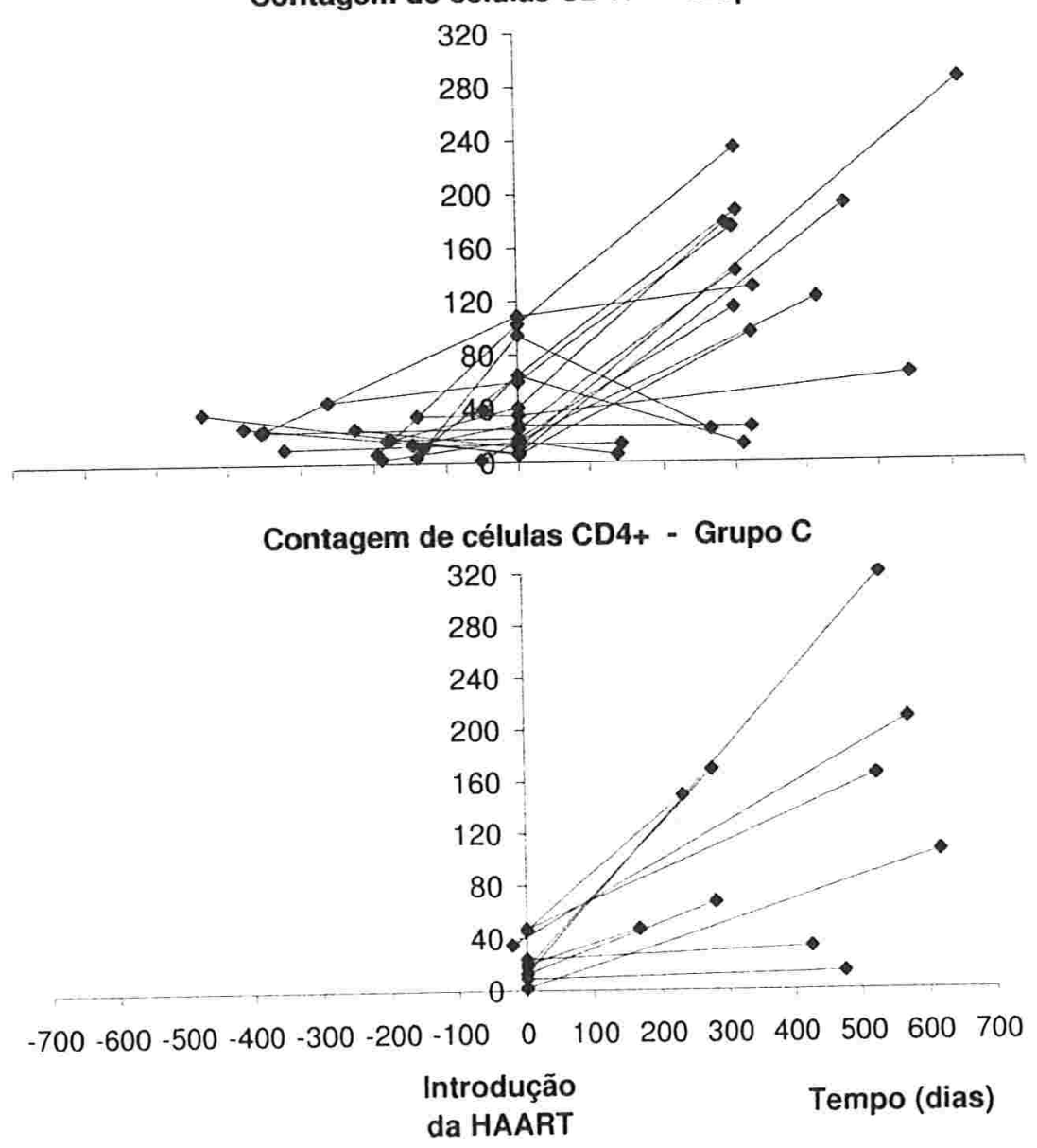

Figura 1.3: Perfis individuais da contagem de células CD4t em pacientes com AIDS. 


\section{Capítulo 2}

\section{Modelo elíptico linear misto}

\subsection{Definição do modelo}

O modelo linear misto proposto por Laird e Ware (1982) assume a forma estrutural

$$
\mathbf{y}_{i}=\mathbf{X}_{i} \boldsymbol{\beta}+\mathbf{Z}_{i} \mathbf{b}_{i}+\boldsymbol{\epsilon}_{i}, i=1, \ldots, n,
$$

em que $\mathbf{y}_{i}$ é um vetor de observações $m_{i}$-dimensional do $i$-ésimo "cluster", $\mathbf{X}_{i}$ é uma matriz $m_{i} \times p$ contendo valores de $p$ variáveis explicativas, $\boldsymbol{\beta}$ é o vetor de parâmetros fixos, $\mathbf{Z}_{i}$ é uma matriz $m_{i} \times q$ de planejamento dos efeitos aleatórios $\mathbf{b}_{i}$, que por sua vez representam os erros entre os "clusters", e, $\boldsymbol{\epsilon}_{i}$ é um vetor $m_{i}$-dimensional de erros "intra-cluster":

$$
\left[\begin{array}{c}
y_{i 1} \\
y_{i 2} \\
\vdots \\
y_{i m_{i}}
\end{array}\right]=\left[\begin{array}{cccc}
x_{i 11} & x_{i 12} & \ldots & x_{i 1 p} \\
x_{i 21} & x_{i 22} & \ldots & x_{i 2 p} \\
\vdots & \vdots & \ddots & \vdots \\
x_{i m_{i} 1} & x_{i m_{i} 2} & \ldots & x_{i m_{i} p}
\end{array}\right]\left[\begin{array}{c}
\beta_{1} \\
\beta_{2} \\
\vdots \\
\beta_{p}
\end{array}\right]+\left[\begin{array}{cccc}
z_{i 11} & z_{i 12} & \ldots & z_{i 1 q} \\
z_{i 21} & z_{i 22} & \ldots & z_{i 2 q} \\
\vdots & \vdots & \ddots & \vdots \\
z_{i m_{i} 1} & z_{i m_{i} 2} & \ldots & z_{i m_{i} q}
\end{array}\right]\left[\begin{array}{c}
b_{i 1} \\
b_{i 2} \\
\vdots \\
b_{i q}
\end{array}\right]+\left[\begin{array}{c}
\varepsilon_{i 1} \\
\varepsilon_{i 2} \\
\vdots \\
\varepsilon_{i m_{i}}
\end{array}\right]
$$

Uma formulação hierárquica está implícita nesse modelo, pois estamos assumindo que $\mathbf{y}_{i} \mid \mathbf{b}_{i} \sim \mathrm{N}_{m_{i}}\left(\mathbf{X}_{i} \boldsymbol{\beta}+\mathbf{Z}_{i} \mathbf{b}_{i} ; \sigma^{2} \mathbf{I}_{m_{i}}\right), \mathbf{b}_{i} \sim N_{q}(\mathbf{0} ; \mathbf{D})$ e $\boldsymbol{\epsilon}_{i} \sim N_{m_{i}}\left(\mathbf{0} ; \sigma^{2} \mathbf{I}_{m_{i}}\right)$. com $\mathrm{b}_{i}$ e $\boldsymbol{\epsilon}_{i}$ independentes. Aqui, a distribuição conjunta de $\left(\mathbf{y}_{i}^{T}, \mathbf{b}_{i}^{T}, \boldsymbol{\epsilon}_{i}^{T}\right)^{T}$ é facilmente determinada: 


$$
\left[\begin{array}{c}
\mathbf{y}_{i} \\
\mathbf{b}_{i} \\
\boldsymbol{\epsilon}_{i}
\end{array}\right] \sim \mathbf{N}_{m_{i}+q+m_{i}}\left\{\left(\begin{array}{c}
\mathbf{X}_{i} \boldsymbol{\beta} \\
\mathbf{0} \\
\mathbf{0}
\end{array}\right) ;\left[\begin{array}{ccc}
\mathbf{Z}_{i} \mathbf{D Z}_{i}^{T}+\sigma^{2} \mathbf{I}_{m_{i}} & \mathbf{Z}_{i} \mathbf{D} & \sigma^{2} \mathbf{I}_{m_{i}} \\
\mathbf{D Z}_{i}^{T} & \mathbf{D} & \mathbf{0} \\
\sigma^{2} \mathbf{I}_{m_{i}} & \mathbf{0} & \sigma^{2} \mathbf{I}_{m_{i}}
\end{array}\right]\right\}
$$

A inferência clássica é, usualmente, baseada na função de verossimilhança do modelo marginal $\mathbf{y}_{i} \sim \mathrm{N}_{m_{i}}\left(\mathbf{X}_{i} \boldsymbol{\beta} ; \mathbf{Z}_{i} \mathbf{D} \mathbf{Z}_{i}^{T}+\sigma^{2} \mathbf{I}_{m_{i}}\right)$.

Sob o enfoque hierárquico a matriz $\mathbf{D}$ deve ser positiva-definida, uma vez que representa a matriz de variâncias-covariâncias do efeitos aleatórios. Por outro lado, no modelo marginal somente a matriz $\mathbf{Z}_{i} \mathbf{D} \mathbf{Z}_{i}^{T}+\sigma^{2} \mathbf{I}_{m_{i}}$ deve ser positivadefinida, ou seja, podemos considerar situações em que os componentes de variância da matriz D sejam negativos. Por exemplo, no caso em que incluímos um efeito aleatório no intercepto, a matriz $\mathbf{D}$ terá um único componente de variância, que chamaremos de $\tau$, que será diretamente proporcional ao coeficiente de correlação intra-classe $\left(\frac{\tau}{\tau+\sigma^{2}}\right)$, ou seja, ao coeficiente de correlação entre as observações feitas no mesmo "cluster". Se em um estudo fizer sentido obter uma correlação intra-classe negativa, então, isso implicará em um componente de variância também negativo. Verbeke e Molenberghs (2003) exemplificam essa situação em um estudo sobre recursos alimentares para filhotes em que ocorre competição por alimento. Nesse exemplo, assumindo que os "clusters" são ninhadas, faz sentido admitir que dentro de cada ninhada para que alguns filhotes comam mais, outros devem comer menos, induzindo a uma correlação intra-classe negativa.

A decisão sobre qual modelo adotar deve estar diretamente ligada à interpretação do problema. No modelo hierárquico, entretanto, um cuidado maior é necessário ao se propor testes de hipóteses para avaliar a necessidade de incluir componentes de variância, uma vez que esses se encontram na fronteira do espaço paramétrico. Giampaoli (1999) abordou essa questão para o caso normal. L'ma forma de contornar esse problema é trabalhar com o modelo marginal mantendo a interpretação do modelo hierárquico, ou seja, propor testes de hipóteses no modelo marginal assumindo que os componentes de variância estão restritos a valores não-negativos. 
Outro questionamento que devemos fazer ao adotar o modelo linear misto está relacionado à suposição de normalidade para os erros, pois sabemos que, sob essa suposição, os estimadores de máxima verossimilhança apresentam falta de robustez na presença de observações aberrantes. Uma possível alternativa para lidar com essa deficiência dos estimadores de máxima verossimilhança é assumir distribuições elípticas com caudas mais pesadas do que as da normal para os erros de tal forma a acomodar melhor essas observações. A classe das distribuições elípticas inclui diversas distribuições com caudas mais leves ou mais pesadas do que as da normal, consistindo, portanto, em uma alternativa interessante nessa situação. A Figura 2.1 apresenta exemplos das distribuições t-Student, exponencial potência, logística-I e II univariadas.

Vamos, a partir de agora, utilizar a notação $\mathrm{Y} \sim \mathrm{El}_{m}(\boldsymbol{\mu} ; \boldsymbol{\Sigma} ; \eta)$ para representar o caso em que o vetor de dados Y assume uma distribuição elíptica $m$-variada com média $\mu$, matriz de variâncias-covariâncias proporcional à $\Sigma$ e com parâmetro de forma $\eta$ definido de maneira diferente para cada elíptica. Para o caso geral vamos considerar a notação simplificada $\mathrm{Y} \sim \mathrm{El}_{m}(\boldsymbol{\mu} ; \boldsymbol{\Sigma})$.

Em modelos elípticos lineares mistos duas abordagens podem ser consideradas. A primeira abordagem seria partir da formulação hierárquica assumindo que $\mathbf{y}_{i} \mid \mathbf{b}_{i} \sim \mathrm{El}_{m_{i}}\left(\mathbf{X}_{i} \boldsymbol{\beta}+\mathbf{Z}_{i} \mathbf{b}_{i} ; \sigma^{2} \mathbf{I}_{m_{i}}\right), \mathbf{b}_{i} \sim \mathrm{El}_{q}(\mathbf{0} ; \mathbf{D})$ e $\boldsymbol{\epsilon}_{i} \sim \mathrm{El}_{m_{i}}\left(\mathbf{0} ; \sigma^{2} \mathbf{I}_{m_{i}}\right)$. Nesse caso, a distribuição conjunta de $\left(\mathbf{y}_{i}^{T}, \mathrm{~b}_{i}^{T}, \epsilon_{i}^{T}\right)^{T}$ não é necessariamente elíptica e obter a distribuição marginal da resposta $\mathbf{y}_{i}$ torna-se analiticamente complexo.

Para modelos mistos com erros assumindo distribuição t-Student multivariada Pinheiro, Liu e Wu (2001) trabalharam uma abordagem hierárquica em dois estágios. Eles partiram da composição da distribuição normal e gama para resultar em uma distribuição marginal t-Student multivariada como se segue:

$$
\begin{gathered}
\mathbf{y}_{i} \mid\left(\mathbf{b}_{i}, \delta_{i}\right) \sim \mathrm{N}_{m_{i}}\left(\mathbf{X}_{i} \beta+\mathbf{Z}_{i} \mathbf{b}_{i} ; \frac{1}{\delta_{i}} \sigma^{2} \mathbf{I}_{m_{i}}\right), \\
\mathbf{b}_{i} \mid \delta_{i} \sim \mathrm{N}_{q}\left(0 ; \frac{1}{\delta_{i}} \mathbf{D}\right)
\end{gathered}
$$



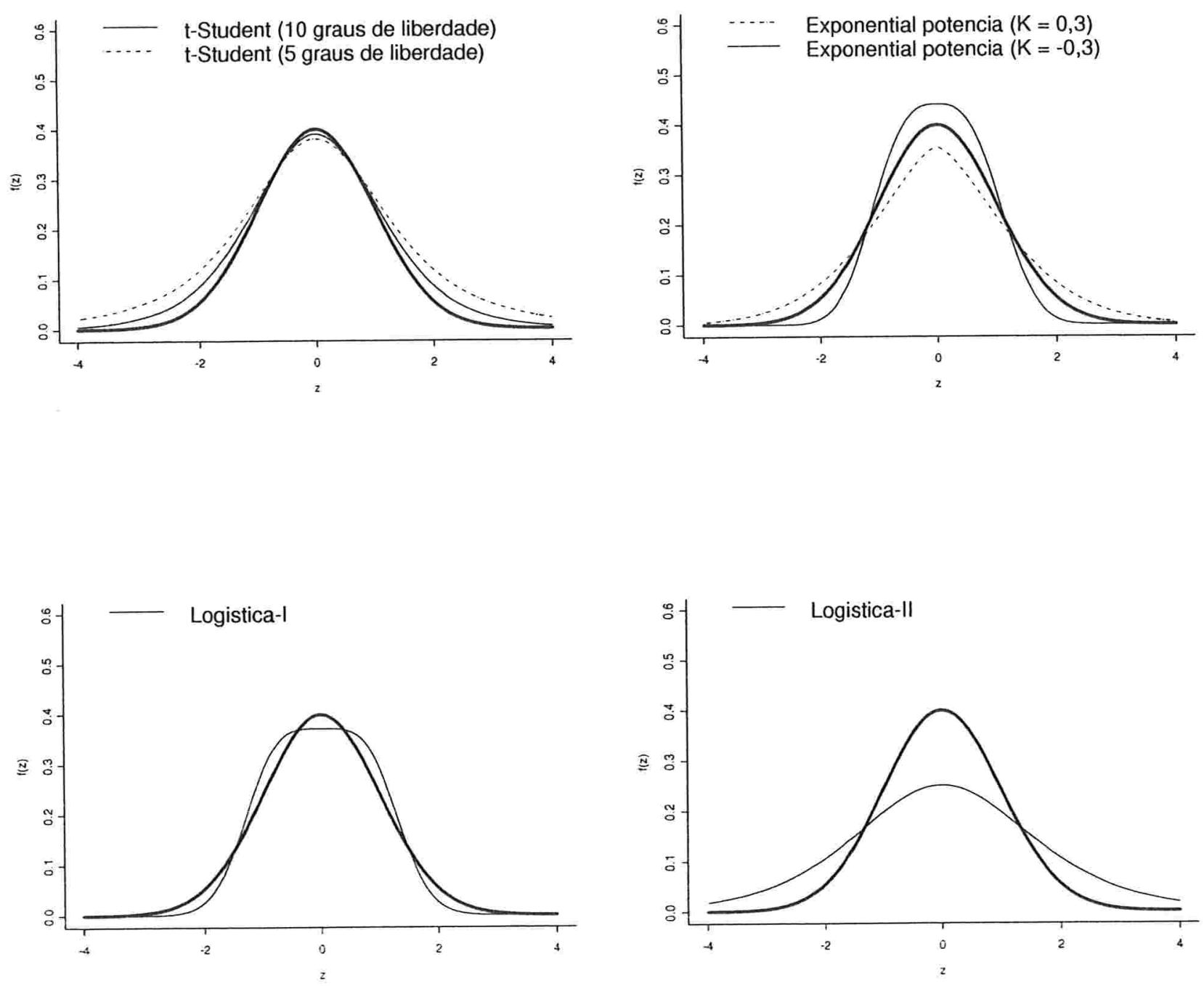

Figura 2.1: Exemplos de algumas distribuições elípticas univariadas comparadas a normal (linha mais cheia). 
e

$$
\delta_{i} \sim \operatorname{Gama}\left(\frac{\nu_{i}}{2} ; \frac{\nu_{i}}{2}\right) \quad i=1, \ldots, n,
$$

que implica em

$$
\mathbf{y}_{i} \sim t_{m_{i}}\left(\mathbf{X}_{i} \boldsymbol{\beta} ; \mathbf{Z}_{i} \mathbf{D} \mathbf{Z}_{i}^{T}+\sigma^{2} \mathbf{I}_{m_{i}} ; \nu_{i}\right)
$$

em que $\nu_{i}$ representa o número de graus de liberdade da distribuição t-Student. Para estimar os parâmetros fixos, aleatórios e graus de liberdade os autores propuseram um processo de estimação baseado no algoritmo EM.

No caso da distribuição t-Student, essa abordagem hierárquica não apresenta grandes dificuldades analíticas, entretanto, para as outras distribuições elípticas, a obtenção da distribuição marginal não é tão simples e pode ser necessário utilizar procedimentos de integração numérica. Assim, a segunda alternativa para evitar as dificuldades analíticas encontradas partindo-se do modelo hierárquico é assumir a mesma estrutura obtida para a conjunta no caso normal apresentada em (2.1.1), mas considerando uma outra distribuição elíptica diretamente para essa conjunta, ou seja,

$$
\left[\begin{array}{c}
\mathbf{y}_{i} \\
\mathbf{b}_{i} \\
\boldsymbol{\epsilon}_{i}
\end{array}\right] \sim \operatorname{El}_{m_{i}+q+m_{i}}\left\{\left(\begin{array}{c}
\mathbf{X}_{i} \boldsymbol{\beta} \\
\mathbf{0} \\
\mathbf{0}
\end{array}\right) ;\left[\begin{array}{ccc}
\mathbf{Z}_{i} \mathbf{D} \mathbf{Z}_{i}^{T}+\sigma^{2} \mathbf{I}_{m_{i}} & \mathbf{Z}_{i} \mathbf{D} & \sigma^{2} \mathbf{I}_{m_{i}} \\
\mathbf{D} \mathbf{Z}_{i}^{T} & \mathbf{D} & \mathbf{0} \\
\sigma^{2} \mathbf{I}_{m_{i}} & \mathbf{0} & \sigma^{2} \mathbf{I}_{m_{i}}
\end{array}\right]\right\}
$$

De acordo com as propriedades das distribuições elípticas, descritas por exemplo em Valle (1994), a distribuição marginal de $\mathbf{y}_{i}$ também será elíptica. Dessa forma, assim como no modelo normal linear misto, as inferências podem ser baseadas na distribuição marginal $\mathbf{y}_{i} \sim \mathrm{El}_{m_{i}}\left(\mathbf{X}_{i} \boldsymbol{\beta} ; \mathbf{Z}_{i} \mathbf{D} \mathbf{Z}_{i}^{T}+\sigma^{2} \mathbf{I}_{m_{i}}\right)$. Observe que sob esse enfoque $\mathbf{b}_{i}$ e $\boldsymbol{\epsilon}_{i}$ são não-correlacionados, mas não necessariamente independentes, exceto no caso normal.

Para essa segunda aborgagem a função densidade de $\mathbf{y}_{i}$ é, então, dada por

$$
f\left(\mathbf{y}_{i}\right)=\left|\Sigma_{i}\right|^{-1 / 2} g\left(u_{i}\right) . \quad i=1, \ldots, n,
$$


em que $u_{i}=\left(\mathbf{y}_{i}-\mu_{i}\right)^{T} \boldsymbol{\Sigma}_{i}^{-1}\left(\mathbf{y}_{i}-\boldsymbol{\mu}_{i}\right)$ é a distância de Mahalanobis e pode ainda ser escrita como $\left(\mathbf{y}_{i}-\boldsymbol{\mu}_{i}\right)^{T} \boldsymbol{\Sigma}_{i}^{-1 / 2} \boldsymbol{\Sigma}_{i}^{-1 / 2}\left(\mathbf{y}_{i}-\boldsymbol{\mu}_{i}\right)=\mathbf{P}_{i}^{T} \mathbf{P}_{i}=\left\|\mathbf{P}_{i}\right\|^{2}$, em que $\left\|\mathbf{P}_{i}\right\|$ é a norma do vetor $\mathbf{P}_{i}$. Adicionalmente $\boldsymbol{\mu}_{i}=\mathbf{X}_{i} \boldsymbol{\beta}$ e $\boldsymbol{\Sigma}_{i}=\mathbf{Z}_{i} \mathbf{D} \mathbf{Z}_{i}^{T}+\sigma^{2} \mathbf{I}_{m_{i}}$. A função $g():. \mathbb{R} \rightarrow[0, \infty]$, chamada função geradora de densidades (veja, por exemplo, Fang, Kotz e Ng, 1990), obedece a condição $\int_{0}^{\infty} u^{m_{i} / 2-1} g(u) d u<\infty$.

Para distribuições elípticas $\mathrm{y}_{i} \sim \mathrm{El}_{m}\left(\boldsymbol{\mu}_{i} ; \boldsymbol{\Sigma}_{i}\right)$, a matriz $\boldsymbol{\Sigma}_{i}$ é proporcional à matriz de variâncias-covariâncias de $\mathbf{y}_{i}$, ou seja, $\operatorname{Var}\left(\mathbf{y}_{i}\right)=\alpha_{i} \boldsymbol{\Sigma}_{i}$. A constante $\alpha_{i}$


para alguma função $\phi$, e é dada por $\alpha_{i}=-2 \phi^{\prime}(0)$, em que $\phi^{\prime}(0)=\left.\frac{\partial \phi^{\prime}(t)}{\partial t}\right|_{t=0}$. Se $\mathbf{y}_{i} \sim \operatorname{El}_{m}\left(\mathbf{0}, \mathbf{I}_{m}\right)$ então $\alpha_{i}$ será a variância das distribuições marginais univariadas. Particularmente, para a distribuição normal $\alpha_{i}=1$.

\subsection{Função escore e matriz de informação de Fisher}

O logaritmo da função de verossimilhanças é dado por

$$
\begin{aligned}
l(\boldsymbol{\theta}) & =\sum_{i=1}^{n} \log \left|\boldsymbol{\Sigma}_{i}\right|^{-1 / 2}+\sum_{i=1}^{n} \log g\left(u_{i}\right) \\
& =\sum_{i=1}^{n}\left[-\frac{1}{2} \log \left|\boldsymbol{\Sigma}_{i}\right|+\log g\left(u_{i}\right)\right] .
\end{aligned}
$$

Considere a matriz de variâncias-covariâncias dos efeitos aleatórios dada por

$$
\mathbf{D}=\left[\begin{array}{cccc}
\tau_{1} & \tau_{12} & \ldots & \tau_{1 q} \\
\tau_{21} & \tau_{2} & \ldots & \tau_{2 q} \\
\vdots & \vdots & \ddots & \vdots \\
\tau_{q 1} & \tau_{q 2} & \ldots & \tau_{q}
\end{array}\right]
$$


Desta forma, o vetor de parâmetros a ser estimado será $\boldsymbol{\theta}=\left(\boldsymbol{\beta}^{T}, \boldsymbol{\gamma}^{T}\right)^{T}$, em que $\gamma_{0}=\sigma^{2}$ e $\gamma_{r}(r=1, \ldots, q+m)$ é o $r$-ésimo elemento do vetor $\gamma$ que contém $q$ parâmetros de variância $\left(\tau_{1}, \ldots, \tau_{q}\right)^{T}$ e $m=\frac{q(q-1)}{2}(q \geq 2)$ parâmetros de covariância entre os efeitos aleatórios $\left(\tau_{12}, \ldots, \tau_{q(q-1)}\right)^{T}$.

Nessas condições, a função escore para $\beta_{k}$ é dada por

$$
\begin{aligned}
S_{\beta_{k}} & =\frac{\partial l(\boldsymbol{\theta})}{\partial \beta_{k}} \\
& =\sum_{i=1}^{n} \frac{g^{\prime}\left(u_{i}\right)}{g\left(u_{i}\right)} \times \frac{\partial u_{i}}{\partial \boldsymbol{\mu}_{i}} \times \frac{\partial \boldsymbol{\mu}_{i}}{\partial \beta_{k}} \\
& =\sum_{i=1}^{n} \frac{g^{\prime}\left(u_{i}\right)}{g\left(u_{i}\right)} \times(-2) \mathbf{x}_{i k}^{T} \boldsymbol{\Sigma}_{i}^{-1}\left(\mathbf{y}_{i}-\boldsymbol{\mu}_{i}\right) \\
& =\sum_{i=1}^{n} v\left(u_{i}\right) \mathbf{x}_{i k}^{T} \Sigma_{i}^{-1}\left(\mathbf{y}_{i}-\boldsymbol{\mu}_{i}\right),
\end{aligned}
$$

em que $\mathbf{x}_{i k}^{T}=\left(x_{i 1 k}, x_{i 2 k}, \ldots, x_{i m_{i} k}\right)^{T}, v\left(u_{i}\right)=-2 w_{g}\left(u_{i}\right), \operatorname{com} w_{g}\left(u_{i}\right)=g^{\prime}\left(u_{i}\right) / g\left(u_{i}\right)$ e $g^{\prime}\left(u_{i}\right)=\partial g\left(u_{i}\right) / \partial u_{i}$, e, $S_{\beta_{k}}$ é o $k$-ésimo elemento do vetor $\mathbf{S}_{\beta}=\left(S_{\beta_{1}}, S_{\beta_{2}}, \ldots, S_{\beta_{p}}\right)^{T}$.

Adicionalmente, a função escore para e $\gamma_{r}$ é dada por

$$
\begin{aligned}
S_{\gamma_{r}} & =\frac{\partial l(\boldsymbol{\theta})}{\partial \gamma_{r}}=-\frac{1}{2} \sum_{i=1}^{n} \frac{\partial}{\partial \gamma_{r}} \log \left|\Sigma_{i}(\gamma)\right|+\sum_{i=1}^{n} \frac{g^{\prime}\left(u_{i}\right)}{g\left(u_{i}\right)} \frac{\partial u_{i}}{\partial \gamma_{r}} \\
& =-\frac{1}{2} \sum_{i=1}^{n} \operatorname{tr}\left[\Sigma_{i}^{-1} \frac{\partial \boldsymbol{\Sigma}_{i}}{\partial \gamma_{r}}\right]+\sum_{i=1}^{n} \frac{g^{\prime}\left(u_{i}\right)}{g\left(u_{i}\right)} \frac{\partial}{\partial \gamma_{r}}\left[\left(\mathbf{y}_{i}-\mu_{i}\right)^{T} \Sigma_{i}^{-1}\left(\mathbf{y}_{i}-\mu_{i}\right)\right] \\
& =-\frac{1}{2} \sum_{i=1}^{n} \operatorname{tr}\left[\Sigma_{i}^{-1} \frac{\partial \Sigma_{i}}{\partial \gamma_{r}}\right]-\frac{1}{2} \sum_{i=1}^{n} v\left(u_{i}\right)\left[\left(\mathbf{y}_{i}-\mu_{i}\right)^{T} \frac{\partial \Sigma_{i}^{-1}}{\partial \gamma_{r}}\left(\mathbf{y}_{i}-\mu_{i}\right)\right]
\end{aligned}
$$




$$
\begin{aligned}
S_{\gamma_{r}} & =-\frac{1}{2} \sum_{i=1}^{n} \operatorname{tr}\left[\boldsymbol{\Sigma}_{i}^{-1} \frac{\partial \boldsymbol{\Sigma}_{i}}{\partial \gamma_{r}}\right]+\frac{1}{2} \sum_{i=1}^{n} v\left(u_{i}\right)\left[\left(\mathbf{y}_{i}-\boldsymbol{\mu}_{i}\right)^{T} \boldsymbol{\Sigma}_{i}^{-1} \frac{\partial \boldsymbol{\Sigma}_{i}}{\partial \gamma_{r}} \boldsymbol{\Sigma}_{i}^{-1}\left(\mathbf{y}_{i}-\boldsymbol{\mu}_{i}\right)\right] \\
& =-\frac{1}{2} \sum_{i=1}^{n}\left\{\operatorname{tr}\left(\boldsymbol{\Sigma}_{i}^{-1} \frac{\partial \boldsymbol{\Sigma}_{i}}{\partial \gamma_{r}}\right)-v\left(u_{i}\right)\left(\mathbf{y}_{i}-\boldsymbol{\mu}_{i}\right)^{T} \boldsymbol{\Sigma}_{i}^{-1} \frac{\partial \boldsymbol{\Sigma}_{i}}{\partial \gamma_{r}} \boldsymbol{\Sigma}_{i}^{-1}\left(\mathbf{y}_{i}-\boldsymbol{\mu}_{i}\right)\right\} .
\end{aligned}
$$

em que $S_{\gamma_{r}}$ é o $r$-ésimo $(r=0, \ldots, q+m)$ elemento do vetor $\mathbf{S}_{\boldsymbol{\gamma}}=\left(S_{\gamma_{0}}, S_{\gamma_{1}}, \ldots, S_{\gamma_{q+m}}\right)^{T}$.

Usamos os resultados de álgebra matricial $\frac{\partial}{\partial x} x^{T} A x=2 x^{T} A, \frac{\partial}{\partial \gamma_{r}} \ln |\Sigma(\gamma)|=$ $\operatorname{tr}\left[\Sigma^{-1} \frac{\partial \Sigma}{\partial \gamma_{r}}\right]$ e $\frac{\partial \Sigma^{-1}(\gamma)}{\partial \gamma_{r}}=-\Sigma^{-1} \frac{\partial \Sigma}{\partial \gamma_{r}} \Sigma^{-1}$ (ver por exemplo, Graybill, 1983).

Para obter a matriz de informação de Fisher para $\boldsymbol{\beta}$ considere:

$$
\begin{gathered}
\frac{\partial l_{i}(\boldsymbol{\theta})}{\partial \boldsymbol{\beta}}=-2 \omega_{g}\left(u_{i}\right) \mathbf{X}_{i}^{T} \boldsymbol{\Sigma}_{i}^{-1}\left(\mathbf{y}_{i}-\boldsymbol{\mu}_{i}\right) \\
=-2 \omega_{g}\left(u_{i}\right) \mathbf{X}_{i}^{T} \boldsymbol{\Sigma}_{i}^{-1 / 2} \boldsymbol{\Sigma}_{i}^{-1 / 2}\left(\mathbf{y}_{i}-\mu_{i}\right)=-2 \omega_{g}\left(u_{i}\right) \mathbf{X}_{i}^{T} \boldsymbol{\Sigma}_{i}^{-1 / 2} \mathbf{P}_{i} \\
\frac{\partial l_{i}(\boldsymbol{\theta}) \frac{\partial l_{i}(\boldsymbol{\theta})}{\partial \beta_{l}}}{\partial \beta_{k}}=\left(-2 \omega_{g}\left(u_{i}\right) \mathbf{x}_{i l}^{T} \boldsymbol{\Sigma}_{i}^{-1 / 2} \mathbf{P}_{i}\right)\left(-2 \omega_{g}\left(u_{i}\right) \mathbf{x}_{i k}^{T} \boldsymbol{\Sigma}_{i}^{-1 / 2} \mathbf{P}_{i}\right) \\
=4 \omega_{g}^{2}\left(u_{i}\right) \mathbf{P}_{i}^{T} \boldsymbol{\Sigma}_{i}^{-1 / 2} \mathbf{x}_{i l} \mathbf{x}_{i k}^{T} \boldsymbol{\Sigma}_{i}^{-1 / 2} \mathbf{P}_{i} \\
=4 \omega_{g}^{2}\left(u_{i}\right)\left\|\mathbf{P}_{i}\right\|^{2} \frac{\mathbf{P}_{i}^{T}}{\left\|\mathbf{P}_{i}\right\|} \boldsymbol{\Sigma}_{i}^{-1 / 2} \mathbf{x}_{i l} \mathbf{x}_{i k}^{T} \boldsymbol{\Sigma}_{i}^{-1 / 2} \frac{\mathbf{P}_{i}^{T}}{\left\|\mathbf{P}_{i}\right\|}
\end{gathered}
$$

em que $\mathbf{x}_{i l}^{T}=\left(x_{i 11}, x_{i 2 l}, \ldots, x_{i m_{i} l}\right)^{T}$ se refere ao $i$-ésimo "cluster" e $l$-ésima variável e $\mathbf{x}_{i k}^{T}=\left(x_{i 1 k}, x_{i 2 k}, \ldots, x_{i m_{k} k}\right)^{T}$ ao $i$-ésimo "cluster" e $k$-ésima variável. 
A partição da matriz de informação de Fisher referente a $\beta_{l}$ e $\beta_{k}$ para o $i$-ésimo "cluster" é dada por

$$
\begin{aligned}
& \mathbf{K}_{\beta_{l} \beta_{k}}=\mathbb{E}\left[\frac{\partial l_{i}(\boldsymbol{\theta})}{\partial \beta_{l}} \frac{\partial l_{i}(\boldsymbol{\theta})}{\partial \beta_{k}}\right]=\mathbb{E}\left\{\mathbb{E}\left[\frac{\partial l_{i}(\boldsymbol{\theta})}{\partial \beta_{l}} \frac{\partial l_{i}(\boldsymbol{\theta})}{\partial \beta_{k}} \mid\left\|\mathbf{P}_{i}\right\|\right]\right\} \\
& \mathbb{E}\left[\frac{\partial l_{i}(\boldsymbol{\theta})}{\partial \beta_{l}} \frac{\partial l_{i}(\boldsymbol{\theta})}{\partial \beta_{k}} \mid\left\|\mathbf{P}_{i}\right\|\right]=\mathbb{E}\left\{4 \omega_{g}^{2}\left(u_{i}\right)\left\|\mathbf{P}_{i}\right\|^{2} \mathbb{E}\left\{\frac{\mathbf{P}_{i}^{T}}{\left\|\mathbf{P}_{i}\right\|} \boldsymbol{\Sigma}_{i}^{-1 / 2} \mathbf{x}_{i l} \mathbf{x}_{i k}^{T} \boldsymbol{\Sigma}_{i}^{-1 / 2} \frac{\mathbf{P}_{i}^{T}}{\left\|\mathbf{P}_{i}\right\|}\right\} \mid\left\|\mathbf{P}_{i}\right\|\right\} \\
&=\mathbb{E}\left\{4 \omega_{g}^{2}\left(u_{i}\right)\left\|\mathbf{P}_{i}\right\|^{2} \frac{1}{m_{i}} \operatorname{tr}\left(\boldsymbol{\Sigma}_{i}^{-1 / 2} \mathbf{x}_{i l} \mathbf{x}_{i k} \boldsymbol{\Sigma}_{i}^{-1 / 2}\right)\right\} \\
&=\frac{1}{m_{i}} \operatorname{tr}\left(\boldsymbol{\Sigma}_{i}^{-1 / 2} \mathbf{x}_{i l} \mathbf{x}_{i k}^{T} \boldsymbol{\Sigma}_{i}^{-1 / 2}\right) \mathbb{E}\left(4 \omega_{g}^{2}\left(u_{i}\right)\left\|\mathbf{P}_{i}\right\|^{2}\right) \\
&=\frac{1}{m_{i}} \operatorname{tr}\left(\mathbf{x}_{i k}^{T} \boldsymbol{\Sigma}_{i}^{-1 / 2} \boldsymbol{\Sigma}_{i}^{-1 / 2} \mathbf{x}_{i l}\right) \mathbb{E}\left(4 \omega_{g}^{2}\left(u_{i}\right)\left\|\mathbf{P}_{i}\right\|^{2}\right) \\
&=\frac{4}{m_{i}} \mathbf{x}_{i k}^{T} \boldsymbol{\Sigma}_{i}^{-1} \mathbf{x}_{i l} \mathbb{E}\left(\omega_{g}^{2}\left(u_{i}\right)\left\|\mathbf{P}_{i}\right\|^{2}\right) \\
&=\frac{4}{m_{i}} \mathbf{x}_{i k}^{T} \boldsymbol{\Sigma}_{i}^{-1} \mathbf{x}_{i l} d_{g_{i}} .
\end{aligned}
$$

Usamos o fato que $\operatorname{tr}(A B)=\operatorname{tr}(B A)$ e o resultado (Graybill, 1983):

$$
\mathbb{E}\left[\frac{\mathbf{P}_{i}^{T}}{\left\|\mathbf{P}_{i}\right\|} \underset{k \times k}{A} \frac{\mathbf{P}_{i}}{\left\|\mathbf{P}_{i}\right\|} \mid\left\|\mathbf{P}_{i}\right\|\right]=\frac{1}{k} \operatorname{tr}(A) .
$$

Portanto, a matriz de informação de Fisher para $\beta$ será dada por

$$
\mathbf{K}_{\beta \beta}=\sum_{i=1}^{n} \frac{4 d_{g_{i}}}{m_{i}} \mathbf{X}_{i}^{T} \boldsymbol{\Sigma}_{i}^{-1} \mathbf{X}_{i}
$$


em que $d_{g_{i}}=\mathbb{E}\left(\omega_{g}^{2}\left(u_{i}\right)\left\|\mathbf{P}_{i}\right\|^{2}\right)$

Para obter a matriz de informação de Fisher para $\gamma$ considere:

$$
\begin{aligned}
& \frac{\partial l_{i}(\boldsymbol{\theta})}{\partial \gamma_{r}}=-\frac{1}{2} \operatorname{tr}\left(\boldsymbol{\Sigma}_{i}^{-1} \frac{\partial \boldsymbol{\Sigma}_{i}}{\partial \gamma_{r}}\right)-\omega_{g}\left(u_{i}\right)\left(\mathbf{y}_{i}-\boldsymbol{\mu}_{i}\right)^{T} \boldsymbol{\Sigma}_{i}^{-1} \frac{\partial \boldsymbol{\Sigma}_{i}}{\partial \gamma_{r}} \boldsymbol{\Sigma}_{i}^{-1}\left(\mathbf{y}_{i}-\boldsymbol{\mu}_{i}\right) \\
& =-\frac{1}{2} \operatorname{tr}\left(\boldsymbol{\Sigma}_{i}^{-1} \frac{\partial \boldsymbol{\Sigma}_{i}}{\partial \gamma_{r}}\right)-\omega_{g}\left(u_{i}\right) \mathbf{P}_{i}^{T} \boldsymbol{\Sigma}_{i}^{-\frac{1}{2}} \frac{\partial \boldsymbol{\Sigma}_{i}}{\partial \gamma_{r}} \boldsymbol{\Sigma}_{i}^{-\frac{1}{2}} \mathbf{P}_{i} \\
& \frac{\partial l_{i}(\boldsymbol{\theta})}{\partial \gamma_{r}} \frac{\partial l_{i}(\boldsymbol{\theta})}{\partial \gamma_{s}}=\frac{1}{4} \operatorname{tr}\left(\boldsymbol{\Sigma}_{i}^{-1} \frac{\partial \boldsymbol{\Sigma}_{i}}{\partial \gamma_{r}}\right) \operatorname{tr}\left(\boldsymbol{\Sigma}_{i}^{-1} \frac{\partial \boldsymbol{\Sigma}_{i}}{\partial \gamma_{s}}\right) \\
& +\frac{1}{2} \operatorname{tr}\left(\boldsymbol{\Sigma}_{i}^{-1} \frac{\partial \boldsymbol{\Sigma}_{i}}{\partial \gamma_{r}}\right) \omega_{g}\left(u_{i}\right) \mathbf{P}_{i}^{T} \boldsymbol{\Sigma}_{i}^{-\frac{1}{2}} \frac{\partial \boldsymbol{\Sigma}_{i}}{\partial \gamma_{s}} \boldsymbol{\Sigma}_{i}^{-\frac{1}{2}} \mathbf{P}_{i} \\
& +\frac{1}{2} \operatorname{tr}\left(\boldsymbol{\Sigma}_{i}^{-1} \frac{\partial \boldsymbol{\Sigma}_{i}}{\partial \gamma_{s}}\right) \omega_{g}\left(u_{i}\right) \mathbf{P}_{i}^{T} \boldsymbol{\Sigma}_{i}^{-\frac{1}{2}} \frac{\partial \boldsymbol{\Sigma}_{i}}{\partial \gamma_{r}} \boldsymbol{\Sigma}_{i}^{-\frac{1}{2}} \mathbf{P}_{i} \\
& +\omega_{g}^{2}\left(u_{i}\right) \mathbf{P}_{i}^{T} \Sigma_{i}^{-\frac{1}{2}} \frac{\partial \boldsymbol{\Sigma}_{i}}{\partial \gamma_{r}} \boldsymbol{\Sigma}_{i}^{-\frac{1}{2}} \mathbf{P}_{i} \mathbf{P}_{i}^{T} \boldsymbol{\Sigma}_{i}^{-\frac{1}{2}} \frac{\partial \boldsymbol{\Sigma}_{i}}{\partial \gamma_{s}} \boldsymbol{\Sigma}_{i}^{-\frac{1}{2}} \mathbf{P}_{i} \\
& =\mathbb{E}\left\{\mathbb{E}\left[(2.2 .4)+(2.2 .5)+(2.2 .6)+(2.2 .7)\|\| \mathbf{P}_{i} \|\right]\right\} .
\end{aligned}
$$

Desenvolvendo cada parcela dessa esperança, obtemos:

$$
\mathbb{E}\left((2.2 .4) \mid\left\|\mathbf{P}_{i}\right\|\right)=\frac{1}{4} \operatorname{tr}\left(\boldsymbol{\Sigma}_{i}^{-1} \frac{\partial \boldsymbol{\Sigma}_{i}}{\partial \gamma_{r}}\right) \operatorname{tr}\left(\boldsymbol{\Sigma}_{i}^{-1} \frac{\partial \boldsymbol{\Sigma}_{i}}{\partial \gamma_{s}}\right),
$$




$$
\mathbb{E}\left[\mathbb{E}\left((2.2 .4) \mid\left\|\mathbf{P}_{i}\right\|\right)\right]=\frac{1}{4} \operatorname{tr}\left(\Sigma_{i}^{-1} \frac{\partial \boldsymbol{\Sigma}_{i}}{\partial \gamma_{r}}\right) \operatorname{tr}\left(\Sigma_{i}^{-1} \frac{\partial \boldsymbol{\Sigma}_{i}}{\partial \gamma_{s}}\right)=\frac{c_{i r s}}{4}
$$

em que

$$
c_{i r s}=\operatorname{tr}\left(\boldsymbol{\Sigma}_{i}^{-1} \frac{\partial \boldsymbol{\Sigma}_{i}}{\partial \gamma_{r}}\right) \operatorname{tr}\left(\boldsymbol{\Sigma}_{i}^{-1} \frac{\partial \boldsymbol{\Sigma}_{i}}{\partial \gamma_{s}}\right)
$$

Usando novamente (2.2.3) temos

$$
\begin{aligned}
\mathbb{E}\left((2.2 .5) \mid\left\|\mathbf{P}_{i}\right\|\right) & =\frac{1}{2} \operatorname{tr}\left(\boldsymbol{\Sigma}_{i}^{-1} \frac{\partial \boldsymbol{\Sigma}_{i}}{\partial \gamma_{r}}\right) \omega_{g}\left(u_{i}\right) \mathbb{E}\left(\mathbf{P}_{i}^{T} \boldsymbol{\Sigma}_{i}^{-\frac{1}{2}} \frac{\partial \boldsymbol{\Sigma}_{i}}{\partial \gamma_{s}} \boldsymbol{\Sigma}_{i}^{-\frac{1}{2}} \mathbf{P}_{i} \mid\left\|\mathbf{P}_{i}\right\|\right) \\
& =\frac{1}{2} \operatorname{tr}\left(\boldsymbol{\Sigma}_{i}^{-1} \frac{\partial \boldsymbol{\Sigma}_{i}}{\partial \gamma_{r}}\right) \omega_{g}\left(u_{i}\right)\left\|\mathbf{P}_{i}\right\|^{2} \mathbb{E}\left(\frac{\mathbf{P}_{i}^{T}}{\left\|\mathbf{P}_{i}\right\|} \boldsymbol{\Sigma}_{i}^{-\frac{1}{2}} \frac{\partial \boldsymbol{\Sigma}_{i}}{\partial \gamma_{s}} \boldsymbol{\Sigma}_{i}^{-\frac{1}{2}} \frac{\mathbf{P}_{i}}{\left\|\mathbf{P}_{i}\right\|} \mid\left\|\mathbf{P}_{i}\right\|\right) \\
& =\frac{1}{2} \operatorname{tr}\left(\boldsymbol{\Sigma}_{i}^{-1} \frac{\partial \boldsymbol{\Sigma}_{i}}{\partial \gamma_{r}}\right) \omega_{g}\left(u_{i}\right)\left\|\mathbf{P}_{i}\right\|^{2} \frac{1}{m_{i}} \operatorname{tr}\left(\boldsymbol{\Sigma}_{i}^{-\frac{1}{2}} \frac{\partial \boldsymbol{\Sigma}_{i}}{\partial \gamma_{s}} \boldsymbol{\Sigma}_{i}^{-\frac{1}{2}}\right)
\end{aligned}
$$

$\mathbb{E}\left[\mathbb{E}\left((2.2 .5) \mid\left\|\mathbf{P}_{i}\right\|\right)\right]=\frac{1}{2 m_{i}} \operatorname{tr}\left(\Sigma_{i}^{-\frac{1}{2}} \frac{\partial \Sigma_{i}}{\partial \gamma_{s}} \Sigma_{i}^{-\frac{1}{2}}\right) \operatorname{tr}\left(\Sigma_{i}^{-1} \frac{\partial \boldsymbol{\Sigma}_{i}}{\partial \gamma_{r}}\right) \mathbb{E}\left(\omega_{g}\left(u_{i}\right)\left\|\mathbf{P}_{i}\right\|^{2}\right)$

$$
\begin{aligned}
& =\frac{1}{2 m_{i}} \operatorname{tr}\left(\Sigma_{i}^{-1} \frac{\partial \boldsymbol{\Sigma}_{i}}{\partial \gamma_{s}}\right) \operatorname{tr}\left(\boldsymbol{\Sigma}_{i}^{-1} \frac{\partial \boldsymbol{\Sigma}_{i}}{\partial \gamma_{r}}\right) \mathbb{E}\left(\omega_{g}\left(u_{i}\right)\left\|\mathbf{P}_{i}\right\|^{2}\right) \\
& =\frac{c_{i r s}}{2 m_{i}} \mathbb{E}\left(\omega_{g}\left(u_{i}\right)\left\|\mathbf{P}_{i}\right\|^{2}\right) .
\end{aligned}
$$

Analogamente,

$$
\mathbb{E}\left[\mathbb{E}\left((2.2 .6) \mid\left\|\mathbf{P}_{i}\right\|\right)\right]=\frac{c_{i r s}}{2 m_{i}} \mathbb{E}\left(\omega_{g}\left(u_{i}\right)\left\|\mathbf{P}_{i}\right\|^{2}\right)
$$


e, por fim,

$$
\begin{aligned}
\mathbb{E} & \left((2.2 .7) \mid\left\|\mathbf{P}_{i}\right\|\right)= \\
= & \omega_{g}^{2}\left(u_{i}\right) \mathbb{E}\left[\mathbf{P}_{i}^{T} \boldsymbol{\Sigma}_{i}^{-\frac{1}{2}} \frac{\partial \boldsymbol{\Sigma}_{i}}{\partial \gamma_{r}} \boldsymbol{\Sigma}_{i}^{-\frac{1}{2}} \mathbf{P}_{i} \mathbf{P}_{i}^{T} \boldsymbol{\Sigma}_{i}^{-\frac{1}{2}} \frac{\partial \boldsymbol{\Sigma}_{i}}{\partial \gamma_{s}} \boldsymbol{\Sigma}_{i}^{-\frac{1}{2}} \mathbf{P}_{i} \mid\left\|\mathbf{P}_{i}\right\|\right] \\
= & \omega_{g}^{2}\left(u_{i}\right)\left\|\mathbf{P}_{i}\right\|^{4} \mathbb{E}\left[\frac{\mathbf{P}_{i}^{T}}{\left\|\mathbf{P}_{i}\right\|}\left(\boldsymbol{\Sigma}_{i}^{-\frac{1}{2}} \frac{\partial \boldsymbol{\Sigma}_{i}}{\partial \gamma_{r}} \boldsymbol{\Sigma}_{i}^{-\frac{1}{2}}\right) \frac{\mathbf{P}_{i}}{\left\|\mathbf{P}_{i}\right\|} \frac{\mathbf{P}_{i}^{T}}{\left\|\mathbf{P}_{i}\right\|}\left(\boldsymbol{\Sigma}_{i}^{-\frac{1}{2}} \frac{\partial \boldsymbol{\Sigma}_{i}}{\partial \gamma_{s}} \boldsymbol{\Sigma}_{i}^{-\frac{1}{2}}\right) \frac{\mathbf{P}_{i}}{\left\|\mathbf{P}_{i}\right\|} \mid\left\|\mathbf{P}_{i}\right\|\right] \\
= & \frac{\omega_{g}^{2}\left(u_{i}\right)\left\|\mathbf{P}_{i}\right\|^{4}}{m_{i}\left(m_{i}+2\right)}\left(2 \operatorname{tr}\left(\boldsymbol{\Sigma}_{i}^{-\frac{1}{2}} \frac{\partial \boldsymbol{\Sigma}_{i}}{\partial \gamma_{r}} \boldsymbol{\Sigma}_{i}^{-1} \frac{\partial \boldsymbol{\Sigma}_{i}}{\partial \gamma_{s}} \boldsymbol{\Sigma}_{i}^{-\frac{1}{2}}\right)+\operatorname{tr}\left(\boldsymbol{\Sigma}_{i}^{-\frac{1}{2}} \frac{\partial \boldsymbol{\Sigma}_{i}}{\partial \gamma_{r}} \boldsymbol{\Sigma}_{i}^{-\frac{1}{2}}\right) \operatorname{tr}\left(\boldsymbol{\Sigma}_{i}^{-\frac{1}{2}} \frac{\partial \boldsymbol{\Sigma}_{i}}{\partial \gamma_{s}} \boldsymbol{\Sigma}_{i}^{-\frac{1}{2}}\right)\right) \\
= & \frac{\omega_{g}^{2}\left(u_{i}\right)\left\|\mathbf{P}_{i}\right\|^{4}}{m_{i}\left(m_{i}+2\right)}\left\{\operatorname{tr}\left(\boldsymbol{\Sigma}_{i}^{-1} \frac{\partial \boldsymbol{\Sigma}_{i}}{\partial \gamma_{r}}\right) \operatorname{tr}\left(\boldsymbol{\Sigma}_{i}^{-1} \frac{\partial \boldsymbol{\Sigma}_{i}}{\partial \gamma_{s}}\right)+2 \operatorname{tr}\left(\boldsymbol{\Sigma}_{i}^{-\frac{1}{2}} \frac{\partial \boldsymbol{\Sigma}_{i}}{\partial \gamma_{r}} \boldsymbol{\Sigma}_{i}^{-1} \frac{\partial \boldsymbol{\Sigma}_{i}}{\partial \gamma_{s}} \boldsymbol{\Sigma}_{i}^{-\frac{1}{2}}\right)\right\},
\end{aligned}
$$

$\mathbb{E}\left[\mathbb{E}\left((2.2 .7) \mid\left\|\mathbf{P}_{i}\right\|\right)\right]=\frac{\mathbb{E}\left(\omega_{g}^{2}\left(u_{i}\right)\left\|\mathbf{P}_{i}\right\|^{4}\right)}{m_{i}\left(m_{i}+2\right)}\left\{c_{i r s}+2 \operatorname{tr}\left(\boldsymbol{\Sigma}_{i}^{-\frac{1}{2}} \frac{\partial \boldsymbol{\Sigma}_{i}}{\partial \gamma_{r}} \boldsymbol{\Sigma}_{i}^{-1} \frac{\partial \boldsymbol{\Sigma}_{i}}{\partial \gamma_{s}} \boldsymbol{\Sigma}_{i}^{-\frac{1}{2}}\right)\right\}$

Usamos o resultado (Graybill, 1983):

$$
\mathbb{E}\left[\frac{\mathbf{P}_{i}^{T}}{\left\|\mathbf{P}_{i}\right\|} A \frac{\mathbf{P}_{i}}{\left\|\mathbf{P}_{i}\right\|} \frac{\mathbf{P}_{i}^{T}}{\left\|\mathbf{P}_{i}\right\|} B \frac{\mathbf{P}_{i}}{\left\|\mathbf{P}_{i}\right\|} \mid\left\|\mathbf{P}_{i}\right\|\right]=\frac{1}{k(k+2)}(2 \operatorname{tr}(A B)+\operatorname{tr}(A) \operatorname{tr}(B)),
$$

em que $A$ e $B$ são matrizes $k \times k$.

Portanto, a partição da matriz de informação de Fisher referente a $\gamma_{r}$ e $\gamma_{s}$ será dada por 


$$
\begin{aligned}
\mathrm{K}_{\gamma_{r} \gamma_{s}}= & \mathbb{E}\left(\frac{\partial l_{i}(\boldsymbol{\theta})}{\partial \gamma_{r}} \frac{\partial l_{i}(\boldsymbol{\theta})}{\partial \gamma_{s}}\right) \\
= & \frac{c_{i r s}}{4}+\frac{c_{i r s}}{m_{i}} \mathbb{E}\left(\omega_{g}\left(u_{i}\right)\left\|\mathbf{P}_{i}\right\|^{2}\right) \\
& +\frac{\mathbb{E}\left(\omega_{g}^{2}\left(u_{i}\right)\left\|\mathbf{P}_{i}\right\|^{4}\right)}{m_{i}\left(m_{i}+2\right)}\left\{c_{i r s}+2 \operatorname{tr}\left(\boldsymbol{\Sigma}_{i}^{-1} \frac{\partial \boldsymbol{\Sigma}_{i}}{\partial \gamma_{r}} \boldsymbol{\Sigma}_{i}^{-1} \frac{\partial \boldsymbol{\Sigma}_{i}}{\partial \gamma_{s}}\right)\right\} .
\end{aligned}
$$

Como, $\mathbb{E}\left(\omega_{g}\left(u_{i}\right)\left\|\mathbf{P}_{i}\right\|^{2}\right)=-\frac{m_{i}}{2}$, ver Fang, Kotz e Ng (1999), então

$$
\mathrm{K}_{\gamma_{r} \gamma_{s}}=\sum_{i=1}^{n}\left[-\frac{c_{i r s}}{4}+\frac{f_{g_{i}}}{m_{i}\left(m_{i}+2\right)}\left\{c_{i r s}+2 \operatorname{tr}\left(\Sigma_{i}^{-1} \frac{\partial \boldsymbol{\Sigma}_{i}}{\partial \gamma_{r}} \boldsymbol{\Sigma}_{i}^{-1} \frac{\partial \boldsymbol{\Sigma}_{i}}{\partial \gamma_{s}}\right)\right\}\right]
$$

é o $(r, s)$-ésimo componente da matriz $\mathbf{K}_{\gamma}, \operatorname{com} f_{g_{i}}=\mathbb{E}\left(\omega_{g}^{2}\left(u_{i}\right)\left\|\mathbf{P}_{i}\right\|^{4}\right)=\mathbb{E}\left\{\omega_{g}^{2}\left(u_{i}\right) u_{i}^{2}\right\}$.

Observe que $\boldsymbol{\beta}$ e $\boldsymbol{\gamma}$ são ortogonais:

$$
\begin{aligned}
\frac{\partial^{2} l_{i}(\boldsymbol{\theta})}{\partial \beta_{k} \partial \gamma_{r}} & =\frac{\partial}{\partial \gamma_{r}}\left\{-2 \omega_{g}\left(u_{i}\right) \mathbf{x}_{i k}^{T} \Sigma_{i}^{-1}\left(\mathbf{y}_{i}-\mu_{i}\right)\right\} \\
& =-2 \omega_{g}\left(u_{i}\right) \mathbf{x}_{i k}^{T} \frac{\partial \boldsymbol{\Sigma}_{i}^{-1}}{\partial \gamma_{r}}\left(\mathbf{y}_{i}-\boldsymbol{\mu}_{i}\right) \\
& =-2 \omega_{g}\left(u_{i}\right) \mathbf{x}_{i k}^{T} \boldsymbol{\Sigma}_{i}^{-1} \frac{\partial \Sigma_{i}}{\partial \gamma_{r}} \Sigma_{i}^{-1}\left(\mathbf{y}_{i}-\boldsymbol{\mu}_{i}\right),
\end{aligned}
$$

e, portanto

$$
\begin{aligned}
\mathbb{E}\left(\frac{\partial^{2} l_{i}(\boldsymbol{\theta})}{\partial \boldsymbol{\beta}_{k} \partial \gamma_{r}}\right) & =\mathbb{E}\left[\mathbb{E}\left(\frac{\partial^{2} l_{i}(\boldsymbol{\theta})}{\partial \boldsymbol{\beta}_{k} \partial \boldsymbol{\gamma}_{r}} \mid\left\|\mathbf{P}_{i}\right\|\right)\right] \\
& \left.=-2 \omega_{g}\left(u_{i}\right) \mathbf{x}_{i k}^{T} \boldsymbol{\Sigma}_{i}^{-1} \frac{\partial \boldsymbol{\Sigma}_{i}}{\partial \gamma_{r}} \boldsymbol{\Sigma}_{i}^{-1} \mathbb{E}\left[\left(\mathbf{y}_{i}-\boldsymbol{\mu}_{i}\right)\right] \mid\left\|\mathbf{P}_{i}\right\|\right)=0
\end{aligned}
$$


Entretanto, $\sigma^{2}$ e $\tau$ não são parâmetros necessariamente ortogonais como veremos mais adiante.

\subsection{Estimação de máxima verossimilhança}

As estimativas de máxima verossimilhança dos parâmetros fixos e dos componentes de variância são obtidas maximizando (2.2.1) simultaneamente com respeito à $\beta$ e $\gamma$.

A solução da equação $\mathbf{S}_{\beta}=0$ é dada por:

$$
\begin{aligned}
& \sum_{i=1}^{n}-2 \omega_{g}\left(\widehat{u}_{i}\right) \mathbf{X}_{i}^{T} \widehat{\boldsymbol{\Sigma}}_{i}^{-1}\left(\mathbf{y}_{i}-\widehat{\boldsymbol{\mu}}_{i}\right)=0 \\
& \sum_{i=1}^{n} v\left(\widehat{u}_{i}\right) \mathbf{X}_{i}^{T} \widehat{\boldsymbol{\Sigma}}_{i}^{-1} \mathbf{y}_{i}-\sum_{i=1}^{n} v\left(\widehat{u}_{i}\right) \mathbf{X}_{i}^{T} \widehat{\boldsymbol{\Sigma}}_{i}^{-1} \mathbf{X}_{i} \widehat{\boldsymbol{\beta}}=0 \\
& \widehat{\boldsymbol{\beta}}=\left[\sum_{i=1}^{n} v\left(\widehat{u}_{i}\right) \mathbf{X}_{i}^{T} \widehat{\boldsymbol{\Sigma}}_{i}^{-1} \mathbf{X}_{i}\right]^{-1} \sum_{i=1}^{n} v\left(\widehat{u}_{i}\right) \mathbf{X}_{i}^{T} \widehat{\boldsymbol{\Sigma}}_{i}^{-1} \mathbf{y}_{i} .
\end{aligned}
$$

Assim, o processo iterativo para estimar os parâmetros fixos e os componentes de variância deve alternar os passos:

$$
\boldsymbol{\beta}^{(r+1)}=\left[\sum_{i=1}^{n} v\left(u_{i}\right)^{(r)} \mathbf{X}_{i}^{T} \boldsymbol{\Sigma}_{i}^{-(r)} \mathbf{X}_{i}\right]^{-1}\left[\sum_{i=1}^{n} v\left(u_{i}\right)^{(r)} \mathbf{X}_{i}^{T} \boldsymbol{\Sigma}_{i}^{-(r)} \mathbf{y}_{i}\right]
$$

e

$$
\boldsymbol{\gamma}^{(r+1)}=\operatorname{argmax}_{\boldsymbol{\gamma}}\left\{l\left(\boldsymbol{\beta}^{(r+1)}, \boldsymbol{\gamma}\right)\right\},
$$

para $r=0,1,2, \ldots$, em que $\boldsymbol{\gamma}=\left(\sigma^{2}, \boldsymbol{\tau}^{T}\right)^{T}$ e $l(\boldsymbol{\beta}, \boldsymbol{\gamma})$ denota o logaritmo da função de verossimilhança. Valores iniciais devem ser fornecidos para inicializar o processo iterativo. Observe que esse processo iterativo é similar ao utilizado 
no modelo marginal assumindo distribuição normal com matriz de variânciascovariâncias dada por $v\left(u_{i}\right) \boldsymbol{\Sigma}_{i}$ e matriz de planejamento $\mathbf{X}_{i}$. Portanto, dados valores iniciais para $\gamma$ e $\beta$ podemos utilizar uma subrotina de modelos lineares mistos para atualizar o vetor de estimativas de $\beta$ e um procedimento de maximização para solucionar a equação (2.3.1). Como valores iniciais sugerimos utilizar os obtidos a partir do modelo normal.

Devido à similaridade entre a inferência nos modelos elípticos e normal, sendo ainda o segundo um caso particular do primeiro, é razoável admitir que, para amostras grandes e sob certas condições de regularidade (Verbeke, 1995), os estimadores de máxima verossimilhança $\hat{\boldsymbol{\beta}}$ e $\hat{\gamma}$ sejam assintoticamente normais com médias $\beta$ e $\gamma$ e matrizes de variâncias-covariâncias $\mathbf{K}_{\beta \beta}^{-1}$ e $\mathbf{K}_{\gamma \gamma}^{-1}$, respectivamente. Kowalski et al. (1999) apresentaram, em particular, alguns resultados assintóticos no caso dos modelos lineares assumindo distribuição t-Student multivariada.

A quantidade $v\left(u_{i}\right)=-2 w_{g}\left(u_{i}\right)$ com $w_{g}\left(u_{i}\right)=g^{\prime}\left(u_{i}\right) / g\left(u_{i}\right)$ que aparece no processo iterativo pode ser interpretada como um peso. De acordo com esse modelo todas as observações de um mesmo "cluster" recebem o mesmo peso. Para exemplificar seguem os cálculos para algumas distribuições elípticas mais importantes.

(i) Distribuição t-Student $\left(\nu_{i}\right)\left(m_{i}\right.$-variada):

$$
\begin{aligned}
& g\left(u_{i}\right)=\underbrace{\frac{\Gamma\left(\frac{m_{i}+\nu_{i}}{2}\right)}{\left(\pi \nu_{i}\right)^{\frac{m_{i}}{2}} \Gamma\left(\frac{\nu_{i}}{2}\right)}\left[1+\frac{1}{\nu_{i}} u_{i}\right]^{-\frac{\nu_{i}+m_{i}}{2}},}_{C} \\
& g^{\prime}\left(u_{i}\right)=C\left(-\frac{\nu_{i}+m_{i}}{2}\right)\left[1+\frac{1}{\nu_{i}} u_{i}\right]^{-\frac{\nu_{i}+m_{i}}{2}-1} \frac{1}{\nu_{i}},
\end{aligned}
$$




$$
\begin{aligned}
w_{g}\left(u_{i}\right) & =\frac{g^{\prime}\left(u_{i}\right)}{g\left(u_{i}\right)}=\frac{C}{C} \frac{-\frac{\nu_{i}+m_{i}}{2}\left[1+\frac{u_{i}}{\nu_{i}}\right]^{-\frac{\nu_{i}+m_{i}}{2}}\left[1+\frac{u_{i}}{\nu_{i}}\right]^{-1} \frac{1}{\nu_{i}}}{\left[1+\frac{u_{i}}{\nu_{i}}\right]^{-\frac{\nu_{i}+m_{i}}{2}}} \\
& =-\left(\frac{\nu_{i}+m_{i}}{2}\right) \frac{1}{\nu_{i}}\left[\frac{\nu_{i}}{\nu_{i}+u_{i}}\right]=-\frac{1}{2}\left(\frac{\nu_{i}+m_{i}}{\nu_{i}+u_{i}}\right),
\end{aligned}
$$

e, portanto,

$$
v\left(u_{i}\right)=-2 w_{g}\left(u_{i}\right)=\frac{\nu_{i}+m_{i}}{\nu_{i}+u_{i}}
$$

(ii) Exponencial Potência $(K)$ :

$$
\begin{gathered}
g\left(u_{i}\right)=C e^{\frac{-u_{i}^{\frac{1}{1+K}}}{2}}, \\
g^{\prime}\left(u_{i}\right)=C e^{\frac{-u_{i}^{\frac{1}{1+K}}}{2}}\left(-\frac{1}{2} \frac{1}{1+K} u_{i}^{\frac{1}{1+K}-1}\right) \\
w_{g}\left(u_{i}\right)=\frac{g^{\prime}\left(u_{i}\right)}{g\left(u_{i}\right)}=\frac{C e^{\frac{-u_{i}^{\frac{1}{2}}}{2}}\left(-\frac{1}{2} \frac{1}{1+K} u_{i}^{\frac{1}{1+K}-1}\right)}{C e^{\frac{-u_{i}^{\frac{1}{1+K}}}{2}}=-\frac{1}{2} \frac{1}{1+K} u_{i}^{\frac{1}{1+K}-1},} \\
\text { portanto, } \\
v\left(u_{i}\right)=-2\left(-\frac{1}{2}\right) \frac{1}{1+K} u_{i}^{\frac{1}{1+K}-1}=\frac{1}{1+K} u_{i}^{\frac{1}{1+K}-1} .
\end{gathered}
$$

e, portanto,

(iii) Logística-I ( $m_{i}$-variada):

$$
g\left(u_{i}\right)=C \frac{e^{-u_{i}}}{\left[1+e^{-u_{i}}\right]^{2}} \quad u_{i} \geq 0
$$




$$
\begin{aligned}
& g^{\prime}\left(u_{i}\right)=C \frac{\left[1+e^{-u_{i}}\right]^{2}\left(-e^{-u_{i}}\right)-e^{-u_{i}} 2\left[1+e^{-u_{i}}\right]\left(-e^{-u_{i}}\right)}{\left[1+e^{-u_{i}}\right]^{4}} \\
& =C \frac{1}{\left(1+e^{-u_{i}}\right)^{4}}\left(-e^{-u_{i}}\right)\left(1+e^{-u_{i}}\right)\left\{\left(1+e^{-u_{i}}\right)-2 e^{-u_{i}}\right\} \\
& =C \frac{\left(-e^{-u_{i}}\right)\left(1+e^{-u_{i}}\right)}{\left(1+e^{-u_{i}}\right)^{4}}\left\{\left(1-e^{-u_{i}}\right)\right\} \\
& =C \frac{\left(-e^{-u_{i}}\right)\left(1+e^{-u_{i}}\right)\left(1-e^{-u_{i}}\right)}{\left(1+e^{-u_{i}}\right)^{4}} \\
& =C \frac{\left(e^{-u_{i}}\right)\left(-1+e^{-u_{i}}\right)}{\left(1+e^{-u_{i}}\right)^{3}}, \\
& w_{g}\left(u_{i}\right)=\frac{g^{\prime}\left(u_{i}\right)}{g\left(u_{i}\right)}=\frac{C \frac{\left(e^{-u_{i}}\right)\left(-1+e^{-u_{i}}\right)}{\left(1+e^{-u_{i}}\right)^{3}}}{\frac{C e^{-u_{i}}}{\left(1+e^{-u_{i}}\right)^{2}}} \\
& =-\frac{\left(1-e^{-u_{i}}\right)}{\left(1+e^{-u_{i}}\right)}=-\tanh \left(\frac{u_{i}}{2}\right)
\end{aligned}
$$

e, portanto,

$$
v\left(u_{i}\right)=2 \tanh \left(\frac{u_{i}}{2}\right)
$$

pois

$$
\tanh z=\frac{e^{z}-e^{-z}}{e^{z}+e^{-z}}=\frac{\frac{e^{z}-e^{-z}}{e^{z}}}{\frac{e^{z}+e^{-z}}{e^{z}}}=\frac{1-e^{-2 z}}{1+e^{-2 z}}
$$

(iv) Logística-II ( $m_{i}$-variada):

$$
g\left(u_{i}\right)=\frac{e^{-u_{i}^{1 / 2}}}{\left[1+e^{-u_{i}^{1 / 2}}\right]^{2}} \quad u_{i} \geq 0
$$


$g^{\prime}\left(u_{i}\right)=\frac{\left[1+e^{-u_{i}^{1 / 2}}\right]^{2}\left(-\frac{1}{2} u_{i}^{-1 / 2} e^{-u_{i}^{1 / 2}}\right)-e^{-u_{i}^{1 / 2}} 2\left[1+e^{-u_{i}^{1 / 2}}\right]\left(-\frac{1}{2} u_{i}^{-1 / 2} e^{-u_{i}^{1 / 2}}\right)}{\left[1+e^{-u_{i}^{1 / 2}}\right]^{4}}$

$$
\begin{aligned}
& w_{g}\left(u_{i}\right)=\frac{g^{\prime}\left(u_{i}\right)}{g\left(u_{i}\right)}=\frac{\frac{\left[1+e^{-u_{i}^{1 / 2}}\right]^{2}\left(-\frac{1}{2} u_{i}^{-1 / 2} e^{-u_{i}^{1 / 2}}\right)-e^{-u_{i}^{1 / 2}} 2\left[1+e^{-u_{i}^{1 / 2}}\right]\left(-\frac{1}{2} u_{i}^{-1 / 2} e^{-u_{i}^{1 / 2}}\right)}{\left[1+e^{-u_{i}^{1 / 2}}\right]^{4}}}{\frac{e^{-u_{i}^{1 / 2}}}{\left[1+e^{-u_{i}^{1 / 2}}\right]^{2}}} \\
& =\frac{\left[1+e^{-u_{i}^{1 / 2}}\right]\left(-\frac{1}{2} u_{i}^{-1 / 2} e^{-u_{i}^{1 / 2}}\right)-e^{-u_{i}^{1 / 2}} 2\left(-\frac{1}{2} u_{i}^{-1 / 2} e^{-u_{i}^{1 / 2}}\right)}{\left[1+e^{-u_{i}^{1 / 2}}\right] e^{-u_{i}^{1 / 2}}} \\
& =\frac{\left[1+e^{-u_{i}^{1 / 2}}\right]\left(-\frac{1}{2} u_{i}^{-1 / 2}\right)-e^{-u_{i}^{1 / 2}} 2\left(-\frac{1}{2} u_{i}^{-1 / 2}\right)}{\left[1+e^{-u_{i}^{1 / 2}}\right]} \\
& =\frac{\left(-\frac{1}{2} u_{i}^{-1 / 2}\right)\left[1-e^{-u_{i}^{1 / 2}}\right]}{\left[1+e^{-u_{i}^{1 / 2}}\right]} \\
& =\frac{-\left[1-e^{-\sqrt{u_{i}}}\right]}{2 \sqrt{u_{i}}\left[1+e^{-\sqrt{u_{i}}}\right]} \\
& =\frac{e^{-\sqrt{u_{i}}}-1}{2 \sqrt{u_{i}}\left[1+e^{-\sqrt{u_{i}}}\right]}
\end{aligned}
$$

e, portanto,

$$
v\left(u_{i}\right)=-\frac{e^{-\sqrt{u_{i}}}-1}{\sqrt{u_{i}}\left[1+e^{-\sqrt{u_{i}}}\right]} .
$$

A Tabela 2.1 resume esses resultados. Por exemplo, para a distribuição tStudent multivariada o peso $v\left(u_{i}\right)$ é inversamente proporcional à distância de Mahalanobis. Assim, quando $u_{i}=\left(\mathbf{y}_{i}-\mathbf{X}_{i} \boldsymbol{\beta}\right)^{T} \boldsymbol{\Sigma}_{i}^{-1}\left(\mathbf{y}_{i}-\mathbf{X}_{i} \boldsymbol{\beta}\right)$ apresenta valor grande, o peso $v\left(u_{i}\right)$ é pequeno, e o procedimento de estimação tende a atribuir pesos pequenos para observações aberrantes. No caso da distribuição exponencial 
Tabela 2.1: Expressões $v\left(u_{i}\right)$ para algumas distribuições elípticas.

\begin{tabular}{cc}
\hline Distribuição & $v\left(u_{i}\right)$ \\
\hline Normal & 1 \\
t-Student $\left(\nu_{i}\right.$ g.l. $)$ & $\frac{\nu_{i}+m_{i}}{\nu_{i}+u_{i}}$ \\
Exponential potência $(K)$ & $\frac{1}{1+K} u_{i}^{\frac{1}{1+K}-1}$ \\
Logística-I & $2 \tanh \left(\frac{u_{i}}{2}\right)$ \\
Logística-II & $-\frac{e^{-\sqrt{u_{i}}}-1}{\sqrt{u_{i}\left[1+e^{-\sqrt{u_{i}}}\right]}}$ \\
\hline
\end{tabular}

potência, o parâmetro $K$ é uma medida de curtose; para $-1<K<0$ a distribuição tem caudas mais leves do que as da normal e para $0<K<1$ a distribuição tem caudas mais pesadas. Quando $K=0$ recaímos na distribuição normal, e portanto esse parâmetro também pode ser visto como um parâmetro de normalidade. Assim, para o objetivo de acomodar observações aberrantes pode-se utilizar $0<K<1$. A distribuição Logística-II também apresenta caudas pesadas, podendo assim ser utilizada para dados com observações aberrantes, enquanto que a distribuição Logística-I apresenta caudas mais leves do que as da normal, podendo ser utilizada para outras finalidades de modelagem.

\subsection{Estimação dos efeitos aleatórios}

A classe de distribuições elípticas multivariadas apresenta uma propriedade útil para obtermos a distribuição a posteriori de $\mathbf{b}_{i}$, dado o vetor de dados observados $\mathbf{y}_{i}$ (veja, por exemplo, Fang, Kotz e Ng, 1990). Utilizando uma notação geral, considere um modelo multivariado assumindo uma distribuição elíptica para o vetor de variáveis de interesse $\mathrm{Y} \sim \mathrm{El}_{m+q}(\boldsymbol{\mu} ; \boldsymbol{\Sigma})$. Adicionalmente, considere que esse vetor possa ser subdividido, induzindo a partição no vetor de médias 
e matriz de variâncias-covariâncias conforme apresentado a seguir:

$$
\mathbf{Y}=\left(\begin{array}{l}
\mathbf{Y}_{1} \\
\mathbf{Y}_{2}
\end{array}\right)_{m+q}, \quad \boldsymbol{\mu}=\left(\begin{array}{l}
\mu_{1} \\
\mu_{2}
\end{array}\right)_{m+q} \text { e } \quad \boldsymbol{\Sigma}=\left[\begin{array}{ll}
\Sigma_{11} & \Sigma_{12} \\
\Sigma_{21} & \Sigma_{22}
\end{array}\right]_{(m+q) \times(m+q)}
$$

Nessas condições, as distribuições condicionais $\mathbf{Y}_{1} \mid \mathbf{Y}_{2}=\mathbf{y}_{2}$ e $\mathbf{Y}_{2} \mid \mathbf{Y}_{1}=\mathbf{y}_{1}$ também são elípticas:

$$
\left(\mathbf{Y}_{1} \mid \mathbf{Y}_{2}=\mathbf{y}_{2}\right) \sim E l_{m}\left(\boldsymbol{\mu}_{1}\left(\mathbf{y}_{2}\right) ; \boldsymbol{\Sigma}_{11.2}\right)
$$

com

$$
\begin{gathered}
\mu_{1}\left(\mathrm{y}_{2}\right)=\mu_{1}+\Sigma_{12} \Sigma_{22}^{-1}\left(\mathrm{y}_{2}-\mu_{2}\right) \\
\Sigma_{11.2}=\Sigma_{11}-\Sigma_{12} \Sigma_{22}^{-1} \Sigma_{21}, \quad \mathrm{e} \\
\left(\mathrm{Y}_{2} \mid \mathrm{Y}_{1}=\mathrm{y}_{1}\right) \sim E l_{q}\left(\mu_{2}\left(\mathrm{y}_{1}\right) ; \Sigma_{22.1}\right)
\end{gathered}
$$

com

$$
\begin{gathered}
\mu_{2}\left(\mathrm{y}_{1}\right)=\mu_{2}+\Sigma_{21} \Sigma_{11}^{-1}\left(\mathrm{y}_{1}-\mu_{1}\right) \\
\Sigma_{22.1}=\Sigma_{22}-\Sigma_{21} \Sigma_{11}^{-1} \Sigma_{12}
\end{gathered}
$$

Portanto, como no caso normal, podemos considerar a distribuição a posteriori de $\mathbf{b}_{i}$ dado o vetor de dados observados $\mathbf{y}_{i}$ para estimar os efeitos aleatórios $\mathbf{b}_{i}$ 's, que também seguem uma distribuição elíptica multivariada. Partindo da conjunta

$$
\left[\begin{array}{l}
\mathbf{y}_{i} \\
\mathbf{b}_{i}
\end{array}\right] \sim \mathrm{El}_{m_{\mathbf{i}}+q}\left\{\left(\begin{array}{c}
\mathbf{X}_{i} \boldsymbol{\beta} \\
0
\end{array}\right) ;\left[\begin{array}{cc}
\mathbf{Z}_{i} \mathbf{D} \mathbf{Z}_{i}^{T}+\sigma^{2} \mathbf{I} & \mathbf{Z}_{i} \mathbf{D} \\
\mathbf{D} \mathbf{Z}_{i}^{T} & \mathbf{D}
\end{array}\right]\right\}
$$


e utilizando as propriedades das distribuições elípticas multivariadas, temos que

$$
\mathbf{b}_{i} \mid \mathbf{y}_{i} \sim \mathrm{El}_{q}\left[\mathbf{0}+\mathbf{D} \mathbf{Z}_{i}^{T} \boldsymbol{\Sigma}_{i}^{-1}\left(\mathbf{y}_{i}-\mathbf{X} \boldsymbol{\beta}\right) ; \mathbf{D}-\mathbf{D Z}_{i}^{T} \boldsymbol{\Sigma}_{i}^{-1} \mathbf{Z}_{i} \mathbf{D}\right]
$$

O vetor de efeitos aleatórios do $i$-ésimo "cluster" que, analogamente ao caso normal, vamos denominar de estimador de Bayes empírico, pode ser estimado pela média da distribuição a posteriori:

$$
\widehat{\mathbf{b}}_{i}=\widehat{\mathbb{E}\left(\mathbf{b}_{i} \mid \mathbf{y}_{i}\right)}=\widehat{\mathbf{D}} \mathbf{Z}_{i}^{T} \widehat{\mathbf{\Sigma}}_{i}^{-1}\left(\mathbf{y}_{i}-\mathbf{X}_{i} \widehat{\boldsymbol{\beta}}\right)
$$

Para obtermos a variância desse estimador, vamos considerar a notação matricial para todos os dados como apresentado a seguir:

$$
\begin{gathered}
\mathbf{Y}=\mathbf{X} \boldsymbol{\beta}+\mathbf{Z} \mathbf{b}+\boldsymbol{\epsilon} \\
{\left[\begin{array}{c}
\mathbf{y}_{1} \\
\mathbf{y}_{2} \\
\vdots \\
\mathbf{y}_{n}
\end{array}\right]_{N \times 1}=\left[\begin{array}{c}
\mathbf{X}_{1} \\
\mathbf{X}_{2} \\
\vdots \\
\mathbf{X}_{n}
\end{array}\right]_{N \times p}\left[\begin{array}{c}
\beta_{1} \\
\beta_{2} \\
\vdots \\
\beta_{p}
\end{array}\right]_{p \times 1}+\left[\begin{array}{cccc}
\mathbf{Z}_{1} & \mathbf{0} & \cdots & \mathbf{0} \\
0 & \mathbf{Z}_{2} & \cdots & 0 \\
\vdots & & \ddots & \vdots \\
\mathbf{0} & 0 & \cdots & \mathbf{Z}_{q}
\end{array}\right]_{N \times n q}\left[\begin{array}{c}
\mathbf{b}_{1} \\
\mathbf{b}_{2} \\
\vdots \\
\mathbf{b}_{q}
\end{array}\right]_{n q \times 1}+\left[\begin{array}{c}
\epsilon_{1} \\
\epsilon_{2} \\
\vdots \\
\epsilon_{n}
\end{array}\right]_{N \times 1}} \\
\text { em que } N=\sum_{i=1}^{n} m_{i} .
\end{gathered}
$$

Supondo $\Sigma_{i}$ e $v\left(u_{i}\right)$ fixos, o estimador de máxima verossimilhança para o vetor de parâmetros $\beta$ é dado por:

$$
\widehat{\boldsymbol{\beta}}=\left[\sum_{i=1}^{n} v\left(u_{i}\right) \mathbf{X}_{i}^{T} \mathbf{W}_{i} \mathbf{X}_{i}\right]^{-1} \sum_{i=1}^{n} v\left(u_{i}\right) \mathbf{X}_{i}^{T} \mathbf{W}_{i} \mathbf{y}_{i}
$$


em que $\mathbf{W}_{i}=\mathbf{\Sigma}_{i}^{-1}$

Chamando $v\left(u_{i}\right)=v_{i}$, podemos expandir $\sum_{i=1}^{n} v_{i} \mathbf{X}_{i}^{T} \mathbf{W}_{i} \mathbf{X}_{i}$ da seguinte forma:

$$
\begin{aligned}
\sum_{i=1}^{n} v_{i} \mathbf{X}_{i}^{T} \mathbf{W}_{i} \mathbf{X}_{i} & =v_{1} \mathbf{X}_{1}^{T} \mathbf{W}_{1} \mathbf{X}_{1}+v_{2} \mathbf{X}_{2}^{T} \mathbf{W}_{2} \mathbf{X}_{2}+\cdots+v_{n} \mathbf{X}_{n}^{T} \mathbf{W}_{n} \mathbf{X}_{n} \\
& =\left[\begin{array}{llll}
\mathbf{X}_{1}^{T} & \mathbf{X}_{2}^{T} & \cdots & \mathbf{X}_{n}^{T}
\end{array}\right]\left[\begin{array}{cccc}
v_{1} \mathbf{W}_{1} & 0 & \cdots & 0 \\
0 & v_{2} \mathbf{W}_{2} & \cdots & 0 \\
\vdots & \vdots & \ddots & \vdots \\
0 & 0 & \cdots & v_{n} \mathbf{W}_{n}
\end{array}\right]\left[\begin{array}{c}
\mathbf{X}_{1} \\
\mathbf{X}_{2} \\
\vdots \\
\mathbf{X}_{n}
\end{array}\right]
\end{aligned}
$$

e denotando

$$
\mathbf{W}^{*}=\operatorname{diag}\left[v_{1} \mathbf{W}_{1}, v_{2} \mathbf{W}_{2}, \ldots, v_{n} \mathbf{W}_{n}\right]
$$

podemos escrever:

$$
\widehat{\boldsymbol{\beta}}=\left(\mathrm{X}^{T} \mathrm{~W}^{*} \mathrm{X}\right)^{-1} \mathrm{X}^{T} \mathrm{~W}^{*} \mathrm{Y}
$$

O vetor completo de efeitos aleatórios também pode ser apresentado utilizando essa notação geral:

$$
\widehat{\mathrm{b}}=\Delta \mathrm{Z}^{T} \mathrm{~W}(\mathrm{Y}-\mathrm{X} \widehat{\boldsymbol{\beta}})
$$

em que $\Delta=\mathbf{D} \otimes \mathbf{I}_{m_{i}}$ e $\mathbf{W}=\operatorname{diag}\left[\mathbf{W}_{1}, \ldots, \mathbf{W}_{n}\right]$.

Assim, a matriz de variâncias-covariâncias de $\widehat{\mathbf{b}}=\left(\widehat{\mathbf{b}}_{1}^{T}, \ldots, \widehat{\mathbf{b}}_{q}^{T}\right)^{T}$ toma a forma

$$
\operatorname{Var}(\widehat{\mathbf{b}})=\underset{n q \times n q}{\Delta} \underset{n q \times N}{\mathbf{Z}^{T}} \underset{N \times N}{\mathbf{W}} \underbrace{\operatorname{Var}(\mathbf{Y}-\mathbf{X} \widehat{\boldsymbol{\beta}})}_{N \times N} \underset{N \times N}{\mathbf{W}} \underset{N \times n q}{\mathbf{Z}} \underset{n q \times n q}{\Delta} .
$$


Considerando a decomposição:

$$
\begin{aligned}
\mathbf{Y}-\mathbf{X} \hat{\boldsymbol{\beta}} & =\mathbf{Y}-\mathbf{X}\left(\mathbf{X}^{T} \mathbf{W}^{*} \mathbf{X}\right)^{-1} \mathbf{X}^{T} \mathbf{W}^{*} \mathbf{Y} \\
& =\left[\mathbf{I}-\mathbf{X}\left(\mathbf{X}^{T} \mathbf{W}^{*} \mathbf{X}\right)^{-1} \mathbf{X}^{T} \mathbf{W}^{*}\right] \mathbf{Y} \\
& =\mathbf{W}^{*^{-1}}[\underbrace{\mathbf{W}^{*}-\mathbf{W}^{*} \mathbf{X}\left(\mathbf{X}^{T} \mathbf{W}^{*} \mathbf{X}\right)^{-1} \mathbf{X}^{T} \mathbf{W}^{*}}_{\mathbf{Q}^{*}}] \mathbf{Y}=\mathbf{W}^{*^{-1}} \mathbf{Q}^{*} \mathbf{Y},
\end{aligned}
$$

temos que

$$
\begin{aligned}
\operatorname{Var}(\mathbf{Y}-\mathbf{X} \widehat{\boldsymbol{\beta}}) & =\mathbf{W}^{*^{-1}} \mathbf{Q}^{*} \operatorname{Var}(\mathbf{Y}) \mathbf{Q}^{*} \mathbf{W}^{*^{-1}} \\
& =\Sigma^{*} \mathbf{Q}^{*} \operatorname{Var}(\mathbf{Y}) \mathbf{Q}^{*} \Sigma^{*}
\end{aligned}
$$

em que $\boldsymbol{\Sigma}^{*}=\operatorname{diag}\left[v_{1} \boldsymbol{\Sigma}_{1}, \ldots, v_{n} \boldsymbol{\Sigma}_{n}\right], \mathbf{Q}^{*}=\left[\boldsymbol{\Sigma}^{*-1}-\boldsymbol{\Sigma}^{*-1} \mathbf{X}\left(\mathbf{X}^{T} \boldsymbol{\Sigma}^{*-1} \mathbf{X}\right)^{-1} \mathbf{X}^{T} \boldsymbol{\Sigma}^{*-1}\right]$ e $\operatorname{Var}(\mathbf{Y})=\operatorname{diag}\left[\alpha_{1} \boldsymbol{\Sigma}_{1}, \ldots, \alpha_{n} \boldsymbol{\Sigma}_{n}\right]$.

Na prática, substitui-se $\boldsymbol{\Sigma}_{i}$ e $v_{i}$ pelas suas estimativas de máxima verossimilhança, e assim, aproximadamente temos:

$$
\operatorname{Var}(\widehat{\mathbf{b}})=\widehat{\Delta} \mathbf{Z}^{T} \widehat{\mathrm{W}} \operatorname{Var}(\mathbf{Y}-\mathbf{X} \widehat{\boldsymbol{\beta}}) \widehat{\mathbf{W}} \mathbf{Z} \widehat{\Delta}
$$

com

$$
\operatorname{Var}(\mathbf{Y}-\mathbf{X} \widehat{\boldsymbol{\beta}})=\widehat{\Sigma}^{*} \widehat{\mathbf{Q}}^{*} \operatorname{Var}(\mathbf{Y}) \widehat{\mathbf{Q}}^{*} \widehat{\Sigma}^{*}
$$

A mesma propriedade, conhecida na literatura como "shrinkage" no caso normal, 
pode ser estendida no caso dos modelos elípticos lineares mistos. O valor ajustado para o $i$-ésimo "cluster" pode ser expresso da seguinte forma:

$$
\begin{aligned}
\widehat{\mathbf{y}}_{i} & =\mathbf{X}_{i} \widehat{\boldsymbol{\beta}}+\mathbf{Z}_{i} \widehat{\mathbf{b}}_{i} \\
& =\mathbf{X}_{i} \widehat{\boldsymbol{\beta}}+\mathbf{Z}_{i} \widehat{\mathbf{D}} \mathbf{Z}_{i}^{T} \widehat{\mathbf{\Sigma}}_{i}^{-1}\left(\mathbf{y}_{i}-\mathbf{X}_{i} \widehat{\boldsymbol{\beta}}\right) \\
& =\left(\mathbf{I}_{m_{i}}-\mathbf{Z}_{i} \widehat{\mathbf{D}} \mathbf{Z}_{i}^{T} \widehat{\mathbf{\Sigma}}_{i}^{-1}\right) \mathbf{X}_{i} \widehat{\boldsymbol{\beta}}+\mathbf{Z}_{i} \widehat{\mathbf{D}} \mathbf{Z}_{i}^{T} \widehat{\boldsymbol{\Sigma}}_{i}^{-1} \mathbf{y}_{i} \\
& =\left(\widehat{\boldsymbol{\Sigma}}_{i}-\mathbf{Z}_{i} \widehat{\mathbf{D}} \mathbf{Z}_{i}^{T}\right) \widehat{\boldsymbol{\Sigma}}_{i}^{-1} \mathbf{X}_{i} \widehat{\boldsymbol{\beta}}+\mathbf{Z}_{i} \widehat{\mathbf{D}} \mathbf{Z}_{i}^{T} \widehat{\boldsymbol{\Sigma}}_{i}^{-1} \mathbf{y}_{i} \\
& =\left(\widehat{\sigma}^{2} \mathbf{I}_{m_{i}}\right) \widehat{\boldsymbol{\Sigma}}_{i}^{-1} \mathbf{X}_{i} \widehat{\boldsymbol{\beta}}+\left(\widehat{\boldsymbol{\Sigma}}_{i}-\widehat{\sigma}^{2} \mathbf{I}_{m_{i}}\right) \widehat{\boldsymbol{\Sigma}}_{i}^{-1} \mathbf{y}_{i} \\
& =\widehat{\sigma}^{2} \widehat{\boldsymbol{\Sigma}}_{i}^{-1} \mathbf{X}_{i} \widehat{\boldsymbol{\beta}}+\left(\mathbf{I}_{m_{i}}-\widehat{\sigma}^{2} \widehat{\boldsymbol{\Sigma}}_{i}^{-1}\right) \mathbf{y}_{i} .
\end{aligned}
$$

A interpretação é a mesma obtida no caso normal. Quando a variabilidade residual $\left(\sigma^{2} \mathbf{I}_{m_{i}}\right)$ for maior do que a variabilidade intra-individual $\left(\boldsymbol{\Sigma}_{i}\right)$, maior peso será dado ao perfil ajustado $\mathbf{X}_{i} \widehat{\boldsymbol{\beta}}$. Por outro lado, quando a variabilidade intraindividual for maior do que a variabilidade residual, maior peso será atribuído para o valor observado $\mathbf{y}_{i}$.

\subsection{Resíduo condicional}

Assumindo a mesma notação matricial adotada na seção 2.4,

$$
\underset{N \times 1}{\mathrm{Y}}=\underset{N \times p}{\mathrm{X}} \underset{p \times 1}{\boldsymbol{\beta}}+\underset{N \times q}{\mathrm{Z}} \underset{q \times 1}{\mathrm{~b}}+\underset{N \times 1}{\epsilon}
$$


podemos definir dois tipos principais de resíduos para estimar o vetor de erros $\epsilon$ em modelos lineares mistos (veja, por exemplo, Verbeke and Molenberghs, 2001, Seção 10.4.4):

i) Resíduo marginal:

$$
\underset{N \times 1}{\widehat{\mathrm{e}}_{M}}=\mathbf{Y}-\widehat{\mathbb{E}(\mathbf{Y})}=\mathbf{Y}-\mathbf{X} \widehat{\boldsymbol{\beta}}
$$

ii) Resíduo condicional:

$$
\left.\underset{N \times 1}{\widehat{\mathbf{e}}_{C}}=\mathbf{Y}-\widehat{\mathbb{E}(\mathbf{Y} \mid \mathbf{b}}\right)=\mathbf{Y}-(\mathbf{X} \widehat{\boldsymbol{\beta}}+\mathbf{Z} \widehat{\mathbf{b}})
$$

Cada resíduo pode ser utilizado para avaliar diferentes aspectos do ajuste do modelo. Esses resíduos, bem como suas propriedades, foram estudados com detalhes no caso normal em Nobre (2003). Para o objetivo deste trabalho, que é identificar observações aberrantes, Nobre (2003) e Hilden-Minton (1995) sugerem utilizar o resíduo condicional, e portanto, vamos aqui trabalhar somente com esse resíduo. Assumindo novamente $\Sigma_{i}$ e $v_{i}$ fixos, o resíduo condicional pode ser escrito como:

$$
\begin{aligned}
\widehat{\mathbf{e}}_{C} & =\mathbf{Y}-\mathbf{X} \widehat{\boldsymbol{\beta}}-\mathbf{Z} \widehat{\mathbf{b}} \\
& =(\mathbf{Y}-\mathbf{X} \widehat{\boldsymbol{\beta}})-\mathbf{Z} \Delta \mathbf{Z}^{T} \mathbf{\Sigma}^{-1}(\mathbf{Y}-\mathbf{X} \widehat{\boldsymbol{\beta}}) \\
& =\left[\mathbf{I}-\mathbf{Z} \Delta \mathbf{Z}^{T} \mathbf{\Sigma}^{-1}\right](\mathbf{Y}-\mathbf{X} \widehat{\boldsymbol{\beta}}) \\
& =\mathbf{A}(\mathbf{Y}-\mathbf{X} \widehat{\boldsymbol{\beta}}),
\end{aligned}
$$


e, portanto, a sua variância é dada por:

$$
\begin{aligned}
\underset{N \times N}{\mathbf{V}_{C}=\operatorname{Var}\left(\widehat{\mathbf{e}}_{C}\right)} & =\mathbf{A} \operatorname{Var}(\mathbf{Y}-\mathbf{X} \widehat{\boldsymbol{\beta}}) \mathbf{A}^{T}, \\
& =\mathbf{A} \boldsymbol{\Sigma}^{*} \mathbf{Q}^{*} \operatorname{Var}(\mathbf{Y}) \mathbf{Q}^{*} \boldsymbol{\Sigma}^{*} \mathbf{A}^{T} .
\end{aligned}
$$

Assim, substituindo $\Sigma_{i}$ e $v_{i}$ pelas suas estimativas de máxima verossimilhança, temos aproximadamente:

$$
\mathrm{V}_{C}=\widehat{\mathbf{A}} \widehat{\Sigma}^{*} \widehat{\mathbf{Q}}^{*} \operatorname{Var}(\mathbf{Y}) \widehat{\mathbf{Q}}^{*} \widehat{\Sigma}^{*} \widehat{\mathbf{A}}^{T}
$$

Como os resíduos podem apresentar variâncias distintas, é conveniente utilizar o resíduo condicional padronizado. Assim, para a l-ésima observação, o resíduo condicional padronizado será estimado por:

$$
\widehat{e}_{C_{l}}^{*}=\frac{\widehat{e}_{C_{l}}}{\sqrt{\widehat{\mathrm{v}}_{C_{l}}}}, l=1 \ldots, N,
$$

em que $\widehat{\mathrm{v}}_{C_{l}}$ é o l-ésimo elemento da diagonal principal da matriz $\widehat{\mathrm{V}}_{C}$ e representa a variância da $l$-ésima observação. Os primeiros $m_{1}$ elementos do vetor $\widehat{\mathrm{e}}_{C}^{*}$ referemse às observações do primeiro "cluster", e assim por diante.

O resíduo condicional padronizado foi utilizado nas aplicações para identificar observações aberrantes no modelo misto normal. No caso das outras distribuições elípticas, esse resíduo também pode ser utilizado, mas deve ser analisado conjuntamente com os pesos $v_{i}$ 's pois observações destacadas como aberrantes podem apresentar um peso pequeno no processo de estimação, não representando, portanto, um problema relevante. 


\subsection{Pontos de alavanca}

Pontos de alavanca são aqueles que exercem influência desproporcional nos próprios valores preditos. No caso normal linear, esses pontos são obtidos pela diagonal principal da matriz de projeção, dada por $\mathbf{X}\left(\mathbf{X}^{T} \mathbf{X}\right)^{-1} \mathbf{X}^{T}$, e representam observações com valores atípicos nas variáveis explicativas. St Laurent e Cook (1992) definiram, para modelos não-lineares, ponto de alavanca como sendo a derivada $\partial \hat{y}_{i} / \partial y_{i}$ e, recentemente, Wei et al (1998) definiram a matriz de alavancagem generalizada para modelos mais gerais.

Assumindo novamente $\Sigma_{i}$ e $v_{i}$ fixos e, utilizando os resultados apresentados em Cysneiros (2004) e Galea, Paula e Cysneiros (2005), podemos aproximar a matriz de alavancagem generalizada para o modelo elíptico linear misto por:

$$
\mathbf{H}=\mathbf{X}\left(\mathbf{X}^{T} \mathbf{W}^{*} \mathbf{X}\right)^{-1} \mathbf{X}^{T} \mathbf{W}^{*}
$$

Como

$$
\begin{aligned}
\operatorname{tr} \mathbf{H} & =\operatorname{tr}\left[\mathbf{X}\left(\mathbf{X}^{T} \mathbf{W}^{*} \mathbf{X}\right)^{-1} \mathbf{X}^{T} \mathbf{W}^{*}\right] \\
& =\operatorname{tr}\left[\left(\mathbf{X}^{T} \mathbf{W}^{*} \mathbf{X}\right)^{-1} \mathbf{X}^{T} \mathbf{W}^{*} \mathbf{X}\right] \\
& =\operatorname{tr} \mathbf{I}_{p}=p,
\end{aligned}
$$

sugerimos utilizar como critério de definição de pontos alavancas o mesmo utilizado em modelos lineares, ou seja, tomar o l-ésimo elemento da diagonal principal de $\mathrm{H}$ e considerar como pontos alavancas aqueles com $h_{l l} \geq 2 p / N$, para $l=1 \ldots \mathrm{V}$.

Adicionalmente, de acordo com Nobre (2003), podemos utilizar

$$
\frac{\sum_{j=1}^{m_{i}} h_{j j}}{m_{i}} \geq \frac{2 p}{N},
$$


para avaliar a alavancagem do $i$-ésimo "cluster".

Nobre (2003) também propôs, para o caso normal, definir uma medida de alavanca considerando a estimação dos efeitos aleatórios, uma vez que a influência dos pontos de alavanca pode também incidir nas predições dos efeitos aleatórios. Assim, generalizando sua proposta para o caso elíptico considere:

$$
\begin{aligned}
\mathbf{H}^{\prime} & =\frac{\partial \widehat{\mathbb{E}(\mathbf{Y} \mid \mathbf{b}})}{\partial \mathbf{Y}^{T}}=\frac{\partial(\mathbf{X} \widehat{\boldsymbol{\beta}}+\mathbf{Z} \widehat{\mathbf{b}})}{\partial \mathbf{Y}^{T}} \\
& =\frac{\partial \mathbf{X} \widehat{\boldsymbol{\beta}}}{\partial \mathbf{Y}^{T}}+\frac{\partial \mathbf{Z} \widehat{\mathbf{b}}}{\partial \mathbf{Y}^{T}} \\
& =\mathbf{H}+\frac{\partial \mathbf{Z} \Delta \mathbf{Z}^{T} \mathbf{W}(\mathbf{Y}-\mathbf{X} \widehat{\boldsymbol{\beta}})}{\partial \mathbf{Y}^{T}} \\
& =\mathbf{H}+\mathbf{Z} \Delta \mathbf{Z}^{T} \mathbf{W} \frac{\partial \mathbf{Y}}{\partial \mathbf{Y}^{T}}-\mathbf{Z} \Delta \mathbf{Z}^{T} \mathbf{W} \frac{\partial \mathbf{X}}{\partial \mathbf{Y}^{T}} \\
& =\mathbf{H}+\mathbf{Z} \Delta \mathbf{Z}^{T} \mathbf{W} \frac{\partial \mathbf{Y}}{\partial \mathbf{Y}^{T}}-\mathbf{Z} \Delta \mathbf{Z}^{T} \mathbf{W H} \\
& =\mathbf{H}+\mathbf{Z} \Delta \mathbf{Z}^{T} \mathbf{W}-\mathbf{Z} \Delta \mathbf{Z}^{T} \mathbf{W}\left(\mathbf{X}\left(\mathbf{X}^{T} \mathbf{W}^{*} \mathbf{X}\right)^{-1} \mathbf{X}^{T} \mathbf{W}^{*}\right) \\
& =\mathbf{H}+\mathbf{Z} \Delta \mathbf{Z}^{T} \mathbf{W} \mathbf{W}^{*-1} \mathbf{Q}^{*} . \\
& =\mathbf{H}+\mathbf{Z} \Delta \mathbf{Z}^{T} \mathbf{W} \mathbf{W}^{*-1}\left(\mathbf{W} \mathbf{W}^{*}-\mathbf{W}^{*} \mathbf{X}\left(\mathbf{X}^{T} \mathbf{W}^{*} \mathbf{X}\right)^{-1} \mathbf{X}^{T} \mathbf{W}^{*}\right) \\
& \mathbf{H}\left(\mathbf{I}_{p}-\mathbf{X}\left(\mathbf{X}^{T} \mathbf{W}^{*} \mathbf{X}\right)^{-1} \mathbf{X}^{T} \mathbf{W}^{*}\right) \\
&
\end{aligned}
$$


Portanto, a l-ésima observação deve ser considerada como alavanca, ou seja, uma observação que exerce forte influência tanto nas estimativas dos efeitos fixos quanto nas dos efeitos aleatórios, se $h_{l l}^{\prime} \geq \frac{2 \operatorname{tr}\left(\mathbf{H}^{\prime}\right)}{N}$. Analogamente ao caso normal, para avaliar a alavancagem do $i$-ésimo "cluster" propomos utilizar:

$$
\frac{\sum_{j=1}^{m_{i}} h_{j j}^{\prime}}{m_{i}} \geq \frac{2 \operatorname{tr}\left(\mathbf{H}^{\prime}\right)}{N}
$$

É importante notar que as observações que recebem pesos $v_{i}$ maiores no processo de estimação, naturalmente serão aquelas que exercerão maior influência nos próprios valores preditos. Assim, uma interpretação mais cuidadosa é necessária para os pontos considerados de alavanca no caso das distribuições elípticas diferentes da normal. Caso o objetivo do estudo seja o de fazer previsões a partir do modelo ajustado, essa ferramenta pode ser utilizada para avaliar se, ao atribuir pesos diferentes às observações, o modelo acaba por perturbar de forma demasiada as predições. Nessas situações, é necessário contrabalançar as vantagens e desvantagens de adotar um modelo elíptico.

\subsection{Escolha da distribuição elíptica}

O problema discutido neste trabalho aplica-se a dados em que existem observações aberrantes. Assim, para esse objetivo, as distribuições elípticas indicadas são aquelas com caudas mais pesadas do que as da normal que acomodam melhor essas observações.

As distribuições elípticas dependem de um parâmetro de forma $\eta$ que define sua curtose e é necessário utilizar um procedimento para escolher um valor para esse parâmetro. Assim, escolhida a distribuição com a qual irá se trabalhar, sugerimos utilizar o critério da informação de Akaike (AIC) (ver, por exemplo, Verbeke and Molenberghs, 2001, p. 74) para escolha desse parâmetro de forma, ainda no modelo de independência.

O critério da informação de Akaike (AIC) compara modelos com base no valor do máximo do logaritmo da verossimilhança penalizado pelo uso de muitos 
parâmetros:

$$
\mathrm{AIC}_{\eta}=-2\left\{l_{\eta}(\boldsymbol{\theta})-p\right\}
$$

em que $p$ é o número de parâmetros fixos no modelo.

A estatística da razão de verossimilhanças baseia-se na diferença entre os logaritmos da função de verossimilhança sob a hipótese alternativa e nula $2\left(l_{1}-l_{0}\right)$. Essa estatística é comparada com uma distribuição qui-quadrado com número graus de liberdade dados pela diferença entre o número de parâmetros sob as duas hipóteses $\left(p_{1}-p_{0}\right)$. De acordo com propriedades dessa distribuição, a hipótese nula pode ser rejeitada se o valor da estatística excede a média da distribuição, dada por $2\left(p_{1}-p_{0}\right)$. Assim, quando $\left(l_{1}-l_{0}\right)>\left(p_{1}-p_{0}\right)$, ou equivalentemente quando $\left(l_{1}-p_{1}\right)>\left(l_{0}-p_{0}\right)$, o modelo com mais parâmetros (sob a hipótese alternativa) é o escolhido. Portanto, ao comparar dois modelos quanto ao AIC, o modelo com menor valor de AIC é o escolhido. Entretanto, vale ressaltar que esse critério não pode ser interpretado como um teste estatístico de significância; ele geralmente é utilizado para comparar modelos com mesma estrutura de médias, mas com diferentes estruturas de dependência, e aqui estamos propondo sua utilização para comparar modelos elípticos sob independência com diferentes parâmetros de forma.

Geralmente, em problemas que utilizam modelos mistos o interesse principal é estimar a tendência média obtida a partir da distribuição marginal. Nos casos em que o principal interesse consiste na estimação específica de cada "cluster", Vaida e Blanchard (2004) discutem ainda a definição de um critério da informação de Akaike que também penalize o valor do máximo do logaritmo da verossimilhança pelo número de efeitos aleatórios estimados, mas para o objetivo de selecionar estruturas de covariância. Como nosso objetivo é utilizar o AIC como critério para escolher o parâmetro de forma da distribuição elíptica no modelo de independência, essa proposta não se aplica. 


\section{Capítulo 3}

\section{Teste para os componentes de variância}

Assumindo o modelo (2.1.2) e supondo inicialmente $\mathbf{D}$ diagonal, é necessário que os parâmetros $\left(\tau_{1}, \ldots, \tau_{q}\right)^{T}$ sejam não-negativos, e, conseqüentemente, a distribuição nula assintótica da estatística da razão de verossimilhanças para testar $\mathrm{H}_{0}: \tau=0$ contra $\mathrm{H}_{1}: \tau>0$, com pelo menos uma desigualdade estrita em $\mathrm{H}_{1}$ não é mais uma qui-quadrado ou mesmo uma mistura de qui-quadrados quando $q \geq 2$. Nesses casos, a hipótese nula está na fronteira do espaço paramétrico, e a inferência clássica não se aplica. Suposições adicionais são necessárias para definir um teste, como já discutido na introdução. Para o caso normal, veja por exemplo, Self e Liang (1987), Vu e Zhou (1997) e Giampaoli (1999). Por outro lado, assumindo o modelo marginal $\mathbf{y}_{i} \sim \mathrm{El}_{m_{i}}\left(\mathbf{X}_{i} \beta ; \mathbf{Z}_{i} \mathrm{DZ}_{i}^{T}+\sigma^{2} \mathbf{I}_{m_{i}}\right)$, a matriz $\mathbf{Z}_{i} \mathbf{D} \mathbf{Z}_{i}^{T}+\sigma^{2} \mathbf{I}_{m_{i}}$ pode ser positiva definida mesmo para valores negativos de $\boldsymbol{\tau}$ e a estatística da razão de verossimilhanças pode ser utilizada para testar $\mathrm{H}_{0}: \tau=\mathbf{0}$ contra $\mathrm{H}_{2}: \boldsymbol{\tau} \neq 0$. Entretanto, estamos interessados em preservar a ligação entre o modelo marginal e o modelo gerador (2.1.2), ou seja, é de nosso interesse manter a hipótese acerca dos componentes de variância unicaudal.

Devido à sua simplicidade, optamos por aplicar o teste do tipo escore proposto por Silvapulle e Silvapulle (1995) para testar $\mathrm{H}_{0}: \tau=0$ contra $\mathrm{H}_{1}: \tau>0$, com pelo menos uma desigualdade estrita em $\mathrm{H}_{1}$. Escolhemos essa estatística principalmente devido ao fato de que ela requer que o modelo seja estimado somente 
sob a hipótese nula, ou seja, sob o modelo mais simples, e essa é uma grande vantagem uma vez que não estão disponíveis muitas ferramentas computacionais para ajustar modelos elípticos lineares mistos, sendo, portanto, uma opção bastante atrativa do ponto de vista computacional. Além disso, essa estatística é assintoticamente equivalente à estatística da razão de verossimilhanças, mesmo no caso restrito, como demonstrado por Silvapulle e Silvapulle (1995). A estatística do tipo escore vem sendo recentemente aplicada em vários contextos, como, por exemplo, para testar hipóteses unicaudais para parâmetros de dispersão. Paula e Artes (2000) utilizaram a estatística do tipo escore para testar a superdispersão em modelos de regressão logística para dados agrupados e Verbeke e Molenberghs (2003) discutem a sua aplicação para testar componentes de variância em modelos lineares mistos. Recentemente, Savalli, Paula e Cysneiros $(2004)^{1}$ desevolveram a estatística do tipo escore para testar componentes de variância em modelos elípticos lineares mistos supondo a matriz D diagonal. Extensões para o caso de D não diagonal são discutidas em Savalli, Paula e Cysneiros (2006).

\subsection{Estatística do tipo escore}

Considere a decomposição da função escore e da matriz de informação de Fisher de acordo com $\boldsymbol{\theta}=\left(\boldsymbol{\lambda}^{T}, \boldsymbol{\tau}^{T}\right)^{T}$, em que $\boldsymbol{\lambda}=\left(\boldsymbol{\beta}^{T}, \sigma^{2}\right)^{T}$ :

$$
\mathrm{S}=\left(\begin{array}{l}
\mathrm{S}_{\lambda} \\
\mathrm{S}_{\tau}
\end{array}\right)
$$

e

$$
\mathbf{K}=\left[\begin{array}{ll}
\mathbf{K}_{\lambda \lambda} & \mathbf{K}_{\lambda \tau} \\
\mathbf{K}_{\tau \lambda} & \mathbf{K}_{\tau \tau}
\end{array}\right]
$$

O parâmetro $\lambda$ é considerado um parâmetro de perturbação para o propósito de

\footnotetext{
${ }^{1}$ Este trabalho recebeu o prêmio "Best Student Presentation Award" no "19th International Workshop on Statistical Modelling" realizado em Florença, Itália em julho de 2004.
} 
testar hipóteses acerca de $\boldsymbol{\tau}$. Silvapulle e Silvapulle (1995) propõem aplicar uma transformação na função escore $S$ de tal forma a obter funções escores para $\tau$ e $\lambda$ não-correlacionadas. Para tanto, considere a transformação AS (Johnson e Wichern, 1992) em que

$$
\mathbf{A}=\left[\begin{array}{cc}
\mathbf{I} & \mathbf{0} \\
-\mathbf{K}_{\tau \lambda} \mathbf{K}_{\lambda \lambda}^{-1} & \mathbf{I}
\end{array}\right]
$$

Nessas condições temos

$$
\mathbf{A S}=\left[\begin{array}{cc}
\mathbf{I} & 0 \\
-\mathbf{K}_{\tau \lambda} \mathbf{K}_{\lambda \lambda}^{-1} & \mathbf{I}
\end{array}\right]\left(\begin{array}{l}
\mathbf{S}_{\lambda} \\
\mathbf{S}_{\tau}
\end{array}\right)=\left(\begin{array}{c}
\mathbf{S}_{\lambda} \\
\mathbf{S}_{\tau}-\mathbf{K}_{\tau \lambda} \mathbf{K}_{\lambda \lambda}^{-1} \mathbf{S}_{\lambda}
\end{array}\right)
$$

e a matriz de variâncias-covariâncias da transformação AS é dada por

$$
\begin{aligned}
\mathbf{A K A}^{T} & =\left[\begin{array}{cc}
\mathbf{I} & \mathbf{0} \\
-\mathbf{K}_{\tau \lambda} \mathbf{K}_{\lambda \lambda}^{-1} & \mathbf{I}
\end{array}\right]\left[\begin{array}{ll}
\mathbf{K}_{\lambda \lambda} & \mathbf{K}_{\lambda \tau} \\
\mathbf{K}_{\tau \lambda} & \mathbf{K}_{\tau \tau}
\end{array}\right]\left[\begin{array}{cc}
\mathbf{I} & \mathbf{0} \\
-\mathbf{K}_{\tau \lambda} \mathbf{K}_{\lambda \lambda}^{-1} & \mathbf{I}
\end{array}\right] \\
& =\left[\begin{array}{cc}
\mathbf{K}_{\lambda \lambda} & \mathbf{0} \\
\mathbf{0} & \mathbf{K}_{\tau \tau}-\mathbf{K}_{\tau \lambda} \mathbf{K}_{\lambda \lambda}^{-1} \mathbf{K}_{\lambda \tau}
\end{array}\right] .
\end{aligned}
$$

Portanto, a função escore $S_{\lambda}$ e a função escore de $\tau$ transformada dada por $\mathbf{S}_{\tau}^{*}=\mathbf{S}_{\tau}-\mathbf{K}_{\tau \lambda} \mathbf{K}_{\lambda \lambda}^{-1} \mathbf{S}_{\lambda}$ apresentam correlação zero, e, podemos assim, utilizar a estatística do tipo escore proposta por Silvapulle e Silvapulle (1995) para testar hipóteses unicaudais acerca de $\tau$ baseadas em $\mathrm{S}_{\tau}^{*}$, de tal forma a eliminar a influência do parâmetro de perturbação $\lambda$.

Assumindo a existência da segunda derivada do logaritmo da função de verossimilhança, e, sob certas condições de regularidade:

i) $n^{-\frac{1}{2}} \mathrm{~S}(\boldsymbol{\theta}) \stackrel{d}{\longrightarrow} \mathrm{N}_{p+q+1}(\mathbf{0} ; \mathbf{K}(\boldsymbol{\theta}))$, 
ii) $\sup _{\|\mathbf{h}\| \leq a}\left[n^{-\frac{1}{2}}\left[\mathbf{S}\left(\boldsymbol{\theta}+n^{-\frac{1}{2}} \mathbf{h}\right)-\mathbf{S}(\boldsymbol{\theta})\right]+\mathbf{K}(\boldsymbol{\theta}) \mathbf{h}\right]=o_{p}(1)$

a estatística do tipo escore é dada por

$$
\mathrm{T}_{S}=\mathrm{S}_{\tau}^{* 0} \mathbf{K}^{\tau \tau 0} \mathbf{S}_{\tau}^{* 0}-\inf _{[\mathbf{a} \geq 0]}\left\{\left(\mathbf{S}_{\tau}^{* 0}-\mathbf{a}\right)^{T} \mathbf{K}^{\tau \tau 0}\left(\mathbf{S}_{\tau}^{* 0}-\mathbf{a}\right)\right\}
$$

em que $\mathbf{K}^{\tau \tau}=\operatorname{Var}(\hat{\tau})$ é obtida subdividindo $\mathbf{K}^{-1}$ e todos os parâmetros são estimados sob a hipótese nula $\widehat{\boldsymbol{\theta}}^{0}=\left(\widehat{\boldsymbol{\beta}}^{0 T}, \widehat{\sigma}_{0}^{2}, \mathbf{0}^{T}\right)^{T}$.

Silvapulle e Silvapulle (1995) mostraram que, para amostras grandes, a estatística da razão de verossimilhanças satisfaz $\mathrm{T}_{R V}=\mathrm{T}_{S}+o_{p}(1)$, e, portanto, elas são assintoticamente equivalentes. Adicionalmente, a distribuição dessa estatística é uma mistura de qui-quadrados centrais:

$$
\mathrm{T}_{S} \stackrel{\mathrm{H}_{0}}{\sim} \sum_{\ell=0}^{q} \omega(\ell ; \Delta) \chi_{\ell}^{2}
$$

em que $\chi_{0}^{2}$ denota a distribuição qui-quadrado degenerada na origem, $\Delta=\operatorname{Var}(\widehat{\tau})$ e $\omega(\ell ; \Delta)$ 's são conhecidos como níveis de probabilidade e são expressos como função dos coeficientes de correlação associados à matriz $\Delta_{q \times q}$ (Shapiro, 1985). Por exemplo, para testar simultaneamente dois componentes de variância com restrição a valores não-negativos segue que, para amostras grandes, $\mathrm{T}_{S} \stackrel{\mathrm{H}_{0}}{\sim} \omega_{0} \chi_{0}^{2}+$ $\omega_{1} \chi_{1}^{2}+\omega_{2} \chi_{2}^{2}$, em que $\omega_{0}=\frac{1}{2 \pi} \cos ^{-1}(\rho), \omega_{1}=\frac{1}{2}$ e $\omega_{2}=\frac{1}{2}-\frac{1}{2 \pi} \cos ^{-1}(\rho)$, com $\rho$ sendo o coeficiente de correlação assintótico entre as estimativas de $\widehat{\tau}_{1}$ e $\widehat{\tau}_{2}$, (Kudô,1963). Para testes com três ou quatro restrições os pesos estão apresentados no apêndice A.

Se $\Delta$ for uma matriz diagonal então as estimativas $\widehat{\tau}_{1}, \widehat{\tau}_{2}, \ldots, \widehat{\tau}_{q}$ são assintoticamente independentes e os níveis de probabilidade tomam a forma

$$
\omega(\ell ; \Delta)=\left(\begin{array}{c}
q \\
\ell
\end{array}\right) 2^{-q}, \ell=0, \ldots, q .
$$


Exemplificando, se o objetivo for testar a inclusão de 2 efeitos aleatórios simultaneamente $(q=2)$, assumindo a matriz $\mathbf{D}$ diagonal, temos as hipóteses $\mathrm{H}_{0}: \tau_{1}=\tau_{2}=0$ com $\mathbf{D}=0$ (modelo de independência) contra $\mathrm{H}_{1}: \tau_{1}>0 \mathrm{e}$ $\tau_{2}>0$ com $\mathbf{D}=\left[\begin{array}{cc}\tau_{1} & 0 \\ 0 & \tau_{2}\end{array}\right]$, então a distribuição nula assintótica da estatística $\mathrm{T}_{S}$ será $\frac{1}{4} \chi_{0}^{2}+\frac{1}{2} \chi_{1}^{2}+\frac{1}{4} \chi_{2}^{2}$.

\subsection{Extensão para matriz D não-diagonal}

Suponha agora que a matriz de variâncias-covariâncias dos efeitos aleatórios D seja não-diagonal com elementos $\tau_{i j}, i, j=1, \ldots, q$ e que o interesse seja testar a existência de $q$ efeitos aleatórios. Esse teste é equivalente a avaliar $\mathrm{H}_{0}: \boldsymbol{\tau}=\mathbf{0}$ contra $\mathrm{H}_{1}: \boldsymbol{\tau}_{1}>0$ e/ou $\boldsymbol{\tau}_{2} \neq \mathbf{0}$, em que $\boldsymbol{\tau}=\left(\boldsymbol{\tau}_{1}^{T}, \boldsymbol{\tau}_{2}^{T}\right)^{T} \operatorname{com} \boldsymbol{\tau}_{1}=\left(\tau_{1}, \ldots, \tau_{q}\right)^{T}$ e $\boldsymbol{\tau}_{2}$ sendo um vetor $m \times 1$ das covariâncias entre os efeitos aleatórios com $m=\frac{q(q-1)}{2}$ $(q \geq 2)$. Nessas condições, a estatística $\mathrm{T}_{S}$ assume a forma

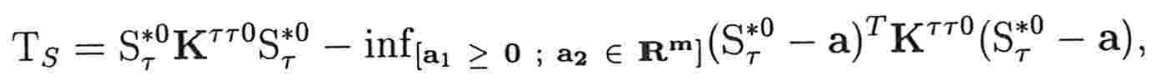

em que a é formado pelos sub-vetores $\mathbf{a}_{1}$ e $\mathbf{a}_{2}$ de dimensões $(q \times 1)$ e $(m \times 1)$, respectivamente, $\mathbf{S}_{\tau}^{*}=\mathbf{S}_{\tau}-\mathbf{K}_{\tau \lambda} \mathbf{K}_{\lambda \lambda}^{-1} \mathbf{S}_{\lambda}$ com $\mathbf{S}_{\tau}$ sendo um vetor de dimensão $(q+m) \times 1$ das funções escores transformadas de $\tau, \mathbf{K}^{\tau \tau}$ é a matriz de variânciascovariâncias assintótica de $\hat{\tau}$ e $\mathbf{K}_{\tau \lambda}$ é a partição $(q+m) \times(p+1)$ da matriz de informação de Fisher. Todas as partições da matriz de informação de Fisher assumem a mesma forma geral $K_{\gamma \gamma}$ apresentada na seção 2.2 e todas as quantidades devem ser estimadas sob a hipótese nula. Novamente para amostras grandes, segue de Silvapulle e Silvapulle (1995, Seção 2) que $T_{S} \stackrel{\mathrm{H}_{0}}{\sim} \sum_{\ell=0}^{q} \omega(\ell ; \Delta) \chi_{\ell+m}^{2}$.

Um caso particular de interesse pode ser testar a existência do q-ésimo efeito aleatório dado que já existem $q-1$ efeitos aleatórios no modelo. Essa hipótese pode ser expressa por $\mathrm{H}_{0}: \tau=0$ contra $\mathrm{H}_{1}: \boldsymbol{\tau}_{1}>0 \mathrm{e} / \mathrm{ou} \tau_{2} \neq 0$, em que 
$\tau_{1}=\tau_{q}$ e $\tau_{2}=\left(\tau_{1 q}, \ldots, \tau_{(q-1) q}\right)^{T}$. A estatística $\mathrm{T}_{S}$ assume a forma acima, e todas as quantidades são estimadas sob a hipótese nula com $(q-1)$ efeitos aleatórios. Nesse caso, para amostras grandes temos que $\mathrm{T}_{S} \stackrel{\mathrm{H}_{0}}{\sim} \frac{1}{2} \chi_{q-1}^{2}+\frac{1}{2} \chi_{q}^{2}$.

Exemplificando, se o objetivo for testar a inclusão de um segundo efeito aleatório $(q=2)$, uma vez que já existe um efeito aleatório no modelo, temos as hipóteses $\mathrm{H}_{0}: \tau_{2}=0$ e $\tau_{12}=0$, com $\mathbf{D}=\tau_{1}$, contra $\mathrm{H}_{1}: \tau_{2}>0$ e $\tau_{12} \neq 0$ com $\mathbf{D}=\left[\begin{array}{cc}\tau_{1} & \tau_{12} \\ \tau_{12} & \tau_{2}\end{array}\right]$, então a distribuição nula assintótica da estatística $\mathrm{T}_{S}$ será $\frac{1}{2} \chi_{1}^{2}+\frac{1}{2} \chi_{2}^{2}$.

Se o objetivo for testar a inclusão de um terceiro efeito aleatório $(q=3)$, uma vez que já existem 2 efeitos aleatórios no modelo, temos as hipóteses $\mathrm{H}_{0}$ : $\boldsymbol{\tau}_{1}=\tau_{3}=0$ e $\boldsymbol{\tau}_{2}=\left(\tau_{13}, \tau_{23}\right)=\mathbf{0}$, com $\mathbf{D}=\left[\begin{array}{cc}\tau_{1} & \tau_{12} \\ \tau_{12} & \tau_{2}\end{array}\right]$ contra $\mathrm{H}_{1}: \boldsymbol{\tau}_{1}=\tau_{3}>0 \mathrm{e}$ $\boldsymbol{\tau}_{2}=\left(\tau_{13}, \tau_{23}\right) \neq \mathbf{0}$ com $\mathbf{D}=\left[\begin{array}{ccc}\tau_{1} & \tau_{12} & \tau_{13} \\ \tau_{12} & \tau_{2} & \tau_{23} \\ \tau_{13} & \tau_{23} & \tau_{3}\end{array}\right]$, então a distribuição nula assintótica da estatística $T_{S}$ será $\frac{1}{2} \chi_{2}^{2}+\frac{1}{2} \chi_{3}^{2}$.

\subsection{Teste para um componente de variância}

Apresentaremos agora a expressão da estatística $T_{S}$ para o caso particular em que o interesse é testar a necessidade de incluir um efeito aleatório no intercepto do modelo. Nessa situação a matriz de variâncias-covariâncias assume a forma $\Sigma_{i}=\tau \mathbf{J}_{m_{i}}+\sigma^{2} \mathbf{I}_{m_{i}}$, e o interesse consiste em testar a hipótese $\mathrm{H}_{0}: \tau=0$ contra $\mathrm{H}_{1}: \tau>0$. As partições da função escore e da matriz de informação de Fisher são dadas, respectivamente, por

$$
\mathrm{S}_{\lambda}=\left(\mathrm{S}_{\beta}^{T}, \mathrm{~S}_{\sigma^{2}}\right)^{T} \text { e } \mathrm{S}_{\tau}=\mathrm{S}_{\tau}
$$

e

$$
\mathbf{K}=\left[\begin{array}{ll}
\mathbf{K}_{\lambda \lambda} & \mathbf{K}_{\lambda \tau} \\
\mathbf{K}_{\tau \lambda} & \mathbf{K}_{\tau \tau}
\end{array}\right]
$$

em que

$$
\mathbf{K}_{\lambda \lambda}=\left[\begin{array}{cc}
\mathbf{K}_{\beta \beta} & \mathbf{K}_{\beta \sigma^{2}} \\
\mathbf{K}_{\sigma^{2} \beta} & \mathbf{K}_{\sigma^{2} \sigma^{2}}
\end{array}\right], \quad \mathbf{K}_{\tau \lambda}=\left[\begin{array}{ll}
\mathbf{K}_{\tau \beta} & \mathbf{K}_{\tau \sigma^{2}}
\end{array}\right] \quad \text { e } \quad \mathbf{K}_{\tau \lambda}=\mathbf{K}_{\tau \lambda}^{T} .
$$


A função escore transformada assume a forma

$$
\begin{aligned}
\mathrm{S}_{\tau}^{*} & =\left[\mathrm{S}_{\tau}-\mathbf{K}_{\tau \lambda} \mathrm{K}_{\lambda \lambda}^{-1} \mathrm{~S}_{\lambda}\right] \\
& =\mathrm{S}_{\tau}-\left[\begin{array}{ll}
\mathbf{0} & \mathrm{K}_{\tau \sigma^{2}}
\end{array}\right]\left[\begin{array}{cc}
\mathrm{K}_{\beta \beta} & \mathbf{0} \\
\mathbf{0} & \mathrm{K}_{\sigma^{2} \sigma^{2}}
\end{array}\right]^{-1}\left(\begin{array}{c}
\mathrm{S}_{\beta} \\
\mathrm{S}_{\sigma^{2}}
\end{array}\right) \\
& =\left[\mathrm{S}_{\tau}-\mathrm{K}_{\tau \sigma^{2}} \mathrm{~K}_{\sigma^{2} \sigma^{2}}^{-1} \mathrm{~S}_{\sigma^{2}}\right] .
\end{aligned}
$$

Analisando a expressão (3.1.1), observe que se $\mathrm{S}_{\tau}^{*}>0$, então inf $\mathrm{ia}_{[\mathrm{a} \geq 0]}\left(\mathrm{S}_{\tau}^{*}-a\right)^{2} \mathrm{~K}^{\tau \tau}=$ 0 com a $=\mathrm{S}_{\tau}^{*}$, entretanto, se $\mathrm{S}_{\tau}^{*} \leq 0$, então $\inf _{[\mathrm{a} \geq 0]}\left(\mathrm{S}_{\tau}^{*}-a\right)^{2} \mathrm{~K}^{\tau \tau}=\left(\mathrm{S}_{\tau}^{*}\right)^{2} \mathrm{~K}^{\tau \tau}$ com $\mathrm{a}=0$. Assim, a expressão de $\mathrm{T}_{S}$ se reduz a

$$
\mathrm{T}_{S}= \begin{cases}\left(\mathrm{S}_{\tau}^{* 0}\right)^{2} \mathrm{~K}^{\tau \tau 0}, & \text { se } \quad \mathrm{S}_{\tau}^{* 0}>0 \\ 0, & \text { se } \quad \mathrm{S}_{\tau}^{* 0} \leq 0\end{cases}
$$

Para desenvolver os cálculos e obter uma expressão para a estatística $T_{S}$ é necessário obter as expressões das funções escore e das matrizes de informação de Fisher. A função escore para o componente de variância $\tau$, obtida a partir da expressão (2.2.2), é dada por

$\mathrm{S}_{\tau}=\frac{\partial l(\boldsymbol{\theta})}{\partial \tau}=-\frac{1}{2} \sum_{i=1}^{n} \operatorname{tr}\left(\boldsymbol{\Sigma}_{i}^{-1} \frac{\partial \boldsymbol{\Sigma}_{i}}{\partial \tau}\right)-\sum_{i=1}^{n} \omega_{g}\left(u_{i}\right)\left(\mathbf{y}_{i}-\boldsymbol{\mu}_{i}\right)^{T} \boldsymbol{\Sigma}_{i}^{-1} \frac{\partial \boldsymbol{\Sigma}_{i}}{\partial \tau} \boldsymbol{\Sigma}_{i}^{-1}\left(\mathbf{y}_{i}-\boldsymbol{\mu}_{i}\right)$,

em que 
$\underset{m_{i} \times m_{i}}{\Sigma_{i}}=\mathbf{J}_{m_{i}} \tau+\mathbf{I}_{m_{i}} \sigma^{2}=\left[\begin{array}{ccccc}\tau+\sigma^{2} & \tau & \tau & \cdots & \tau \\ & \tau+\sigma^{2} & \tau & \cdots & \tau \\ & & \ddots & & \\ & & & & \tau+\sigma^{2}\end{array}\right]$

$$
\begin{aligned}
& \frac{\partial \boldsymbol{\Sigma}_{i}}{\partial \tau}=\left[\begin{array}{ccccc}
1 & 1 & 1 & \cdots & 1 \\
& 1 & 1 & \cdots & 1 \\
& & \ddots & & \\
& & & & 1
\end{array}\right]_{m_{i} \times m_{i}}=\mathbf{J}_{m_{i}} \\
& \underset{m_{i} \times m_{i}}{\Sigma_{i}^{-1}}=\frac{1}{\sigma^{2}}\left[\mathbf{I}_{m_{i}}-\left(\frac{\tau}{m_{i} \tau+\sigma^{2}}\right) \mathbf{J}_{m_{i}}\right], \\
& \boldsymbol{\Sigma}_{i}^{-1} \frac{\partial \boldsymbol{\Sigma}_{i}}{\partial \tau}=\frac{1}{\sigma^{2}}\left[\mathbf{I}_{m_{i}}-\left(\frac{\tau}{m_{i} \tau+\sigma^{2}}\right) \mathbf{J}_{m_{i}}\right] \mathbf{J}_{m_{i}} \\
& =\frac{1}{\sigma^{2}}\left[\mathbf{J}_{m_{i}}-\frac{\tau}{m_{i} \tau+\sigma^{2}} m_{i} \mathbf{J}_{m_{i}}\right] \\
& =\frac{1}{\sigma^{2}} \mathbf{J}_{m_{i}}\left[\mathbf{I}_{m_{i}}-\frac{m_{i} \tau}{m_{i} \tau+\sigma^{2}} \mathbf{I}_{m_{i}}\right] \\
& =\frac{1}{\sigma^{2}} \mathbf{J}_{m_{i}}\left[\mathbf{I}_{m_{i}}\left[1-\frac{m_{i} \tau}{m_{i} \tau+\sigma^{2}}\right]\right] \\
& =\frac{1}{\sigma^{2}} \mathbf{J}_{m_{i}}\left[\mathbf{I}_{m_{i}} \frac{\sigma^{2}}{m_{i} \tau+\sigma^{2}}\right] \\
& =\frac{1}{m_{i} \tau+\sigma^{2}} \mathbf{J}_{m_{i}},
\end{aligned}
$$


o que acarreta

$$
\operatorname{tr}\left(\Sigma_{i}^{-1} \frac{\partial \boldsymbol{\Sigma}_{i}}{\partial \tau}\right)=\operatorname{tr}\left(\frac{1}{m_{i} \tau+\sigma^{2}} \mathbf{J}_{m_{i}}\right)=\frac{m_{i}}{m_{i} \tau+\sigma^{2}}
$$

Adicionalmente,

$$
\begin{aligned}
\left(\boldsymbol{\Sigma}_{i}^{-1} \frac{\partial \boldsymbol{\Sigma}_{i}}{\partial \tau}\right) \boldsymbol{\Sigma}_{i}^{-1} & =\frac{1}{m_{i} \tau+\sigma^{2}} \mathbf{J}_{m_{i}}\left[\frac{1}{\sigma^{2}}\left(\mathbf{I}_{m_{i}}-\frac{\tau}{m_{i} \tau+\sigma^{2}} \mathbf{J}_{m_{i}}\right)\right] \\
& =\frac{1}{m_{i} \tau+\sigma^{2}} \frac{1}{\sigma^{2}}\left[\mathbf{J}_{m_{i}}-\frac{m_{i} \tau}{m_{i} \tau+\sigma^{2}} \mathbf{J}_{m_{i}}\right] \\
& =\frac{1}{m_{i} \tau+\sigma^{2}} \frac{1}{\sigma^{2}} \mathbf{J}_{m_{i}}\left[\mathbf{I}_{m_{i}} \frac{\sigma^{2}}{m_{i} \tau+\sigma^{2}}\right]=\mathbf{J}_{m_{i}}\left(\frac{1}{\left(m_{i} \tau+\sigma^{2}\right)^{2}}\right)
\end{aligned}
$$

Substituindo esses resultados na expressão (3.3.2), a função escore para $\tau$ fica

$$
\begin{aligned}
\mathrm{S}_{\tau} & =-\frac{1}{2} \sum_{i=1}^{n}\left(\frac{m_{i}}{m_{i} \tau+\sigma^{2}}\right)+\sum_{i=1}^{n} \frac{v\left(u_{i}\right)}{2}\left(\mathbf{y}_{i}-\boldsymbol{\mu}_{i}\right)^{T} \frac{\mathbf{J}_{m_{i}}}{\left(m_{i} \tau+\sigma^{2}\right)^{2}}\left(\mathbf{y}_{i}-\boldsymbol{\mu}_{i}\right) \\
& =-\frac{1}{2} \sum_{i=1}^{n}\left(\frac{m_{i}}{m_{i} \tau+\sigma^{2}}\right)+\sum_{i=1}^{n} \frac{v\left(u_{i}\right)}{2} \frac{1}{\left(m_{i} \tau+\sigma^{2}\right)^{2}}\left[\sum_{j=1}^{m_{i}}\left(y_{i j}-\mu_{i j}\right)\right]^{2} \\
& =\sum_{i=1}^{n}\left(\frac{1}{m_{i} \tau+\sigma^{2}}\right)\left[-\frac{m_{i}}{2}+\frac{v\left(u_{i}\right)}{2\left(m_{i} \tau+\sigma^{2}\right)}\left[\sum_{j=1}^{m_{i}}\left(y_{i j}-\mu_{i j}\right)\right]^{2}\right],
\end{aligned}
$$

que, sob $\mathrm{H}_{0}: \tau=0$, resulta 


$$
\mathrm{S}_{\tau}^{0}=\left.\frac{\partial l(\boldsymbol{\theta})}{\partial \tau}\right|_{\tau=0}=\sum_{i=1}^{n}\left\{-\frac{m_{i}}{2 \sigma_{0}^{2}}+\frac{v\left(u_{i}^{0}\right)}{2\left(\sigma_{0}^{2}\right)^{2}}\left[\sum_{j=1}^{m_{i}}\left(y_{i j}-\mu_{i j}^{0}\right)\right]^{2}\right\} .
$$

A função escore para o componente de variância $\sigma^{2}$, também obtida a partir de (2.2.2), é dada por

$\mathrm{S}_{\sigma^{2}}=\frac{\partial l(\boldsymbol{\theta})}{\partial \sigma^{2}}=-\frac{1}{2} \sum_{i=1}^{n} \operatorname{tr}\left(\boldsymbol{\Sigma}_{i}^{-1} \frac{\partial \boldsymbol{\Sigma}_{i}}{\partial \sigma^{2}}\right)-\sum_{i=1}^{n} \omega_{g}\left(u_{i}\right)\left(\mathbf{y}_{i}-\boldsymbol{\mu}_{i}\right)^{T} \Sigma_{i}^{-1} \frac{\partial \boldsymbol{\Sigma}_{i}}{\partial \sigma^{2}} \Sigma_{i}^{-1}\left(\mathbf{y}_{i}-\boldsymbol{\mu}_{i}\right)$,

em que

$$
\begin{aligned}
& \frac{\partial \boldsymbol{\Sigma}_{i}}{\partial \sigma^{2}}=\mathbf{I}_{m_{i}} \\
& \Sigma_{i}^{-1} \frac{\partial \boldsymbol{\Sigma}_{i}}{\partial \sigma^{2}}=\Sigma_{i}^{-1}=\frac{1}{\sigma^{2}}\left[\mathbf{I}_{m_{i}}-\frac{\tau}{m_{i} \tau+\sigma^{2}} \mathbf{J}_{m_{i}}\right] \\
& =\frac{1}{\sigma^{2}}\left[\begin{array}{cccc}
1-\frac{\tau}{m_{i} \tau+\sigma^{2}} & -\frac{\tau}{m_{i} \tau+\sigma^{2}} & \cdots & -\frac{\tau}{m_{i} \tau+\sigma^{2}} \\
& 1-\frac{\tau}{m_{i} \tau+\sigma^{2}} & -\frac{\tau}{m_{i} \tau+\sigma^{2}} & \\
& & \ddots & 1-\frac{\tau}{m_{i} \tau+\sigma^{2}}
\end{array}\right],
\end{aligned}
$$

o que acarreta

$$
\operatorname{tr}\left(\Sigma_{i}^{-1}\right)=\frac{1}{\sigma^{2}} m_{i}\left(1-\frac{\tau}{m_{i} \tau+\sigma^{2}}\right)=\frac{1}{\sigma^{2}} m_{i}\left(\frac{\left(m_{i}-1\right) \tau+\sigma^{2}}{m_{i} \tau+\sigma^{2}}\right) .
$$

Substituindo esses resultados na expressão (3.3.4). a função escore para $\sigma^{2}$ fica 
$\mathrm{S}_{\sigma^{2}}=-\frac{1}{2} \sum_{i=1}^{n} \frac{1}{\sigma^{2}} m_{i}\left(\frac{\left(m_{i}-1\right) \tau+\sigma^{2}}{m_{i} \tau+\sigma^{2}}\right)-\sum_{i=1}^{n} \omega_{g}\left(u_{i}\right)\left(\mathbf{y}_{i}-\boldsymbol{\mu}_{i}\right)^{T} \boldsymbol{\Sigma}_{i}^{-1} \boldsymbol{\Sigma}_{i}^{-1}\left(\mathbf{y}_{i}-\boldsymbol{\mu}_{i}\right)$

em que

$$
\begin{aligned}
\boldsymbol{\Sigma}_{i}^{-1} \boldsymbol{\Sigma}_{i}^{-1} & =\left[\frac{1}{\sigma^{2}}\left(\mathbf{I}_{m_{i}}-\frac{\tau}{m_{i} \tau+\sigma^{2}} \mathbf{J}_{m_{i}}\right)\right]\left[\frac{1}{\sigma^{2}}\left(\mathbf{I}_{m_{i}}-\frac{\tau}{m_{i} \tau+\sigma^{2}} \mathbf{J}_{m_{i}}\right)\right] \\
& =\frac{1}{\sigma^{4}}\left[\mathbf{I}_{m_{i}}-2 \frac{\tau}{m_{i} \tau+\sigma^{2}} \mathbf{J}_{m_{i}}+\left(\frac{\tau}{m_{i} \tau+\sigma^{2}}\right)^{2} m_{i} \mathbf{J}_{m_{i}}\right]
\end{aligned}
$$

e, sob $\mathrm{H}_{0}: \tau=0$, temos $\Sigma_{i}^{-1} \Sigma_{i}^{-1}=\frac{1}{\sigma_{0}^{4}} \mathbf{I}_{m_{i}}$. Segue que a função escore para o componente $\sigma^{2}$ assume a forma

$$
\begin{aligned}
\mathrm{S}_{\sigma^{2}}^{0}=\left.\frac{\partial l(\boldsymbol{\theta})}{\partial \sigma^{2}}\right|_{\tau=0} & =\sum_{i=1}^{n}-\frac{m_{i}}{2 \sigma_{0}^{2}}+\sum_{i=1}^{n} \frac{v\left(u_{i}^{0}\right)}{2 \sigma_{0}^{4}}\left(\mathbf{y}_{i}-\boldsymbol{\mu}_{i}^{0}\right)^{T}\left(\mathbf{y}_{i}-\boldsymbol{\mu}_{i}^{0}\right) \\
& =\sum_{i=1}^{n}-\frac{m_{i}}{2 \sigma_{0}^{2}}+\sum_{i=1}^{n} \frac{v\left(u_{i}^{0}\right)}{2 \sigma_{0}^{4}}\left[\sum_{j=1}^{m_{i}}\left(y_{i j}-\mu_{i j}^{0}\right)^{2}\right] .
\end{aligned}
$$

A matriz de informação de Fisher esperada para $\tau$ e $\sigma^{2}$ é dada por

$$
\left[\begin{array}{cc}
\mathrm{K}_{\sigma^{2} \sigma^{2}} & \mathrm{~K}_{\tau \sigma^{2}} \\
\mathrm{~K}_{\sigma^{2} \tau} & \mathrm{K}_{\tau \tau}
\end{array}\right]
$$

e. como já vimos anteriormente,

$$
\mathrm{K}_{\gamma_{r} \gamma_{s}}=\sum_{i=1}^{n}\left[-\frac{c_{i r s}}{4}+\frac{f_{g_{i}}}{m_{i}\left(m_{i}+2\right)}\left\{c_{i r s}+2 \operatorname{tr}\left(\Sigma_{i}^{-1} \frac{\partial \boldsymbol{\Sigma}_{i}}{\partial \gamma_{r}} \Sigma_{i}^{-1} \frac{\partial \boldsymbol{\Sigma}_{i}}{\partial \gamma_{s}}\right)\right\}\right]
$$


Para o caso da matriz com um único efeito aleatório vamos assumir $\gamma_{0}=\sigma^{2}$ e $\gamma_{1}=\tau$. Como

$$
\operatorname{tr}\left(\Sigma_{i}^{-1} \frac{\partial \Sigma_{i}}{\partial \tau}\right)=\frac{m_{i}}{m_{i} \tau+\sigma^{2}}
$$

temos que

$$
c_{i \tau \tau}=\left(\frac{m_{i}}{m_{i} \tau+\sigma^{2}}\right)^{2}
$$

Além disso,

$$
\boldsymbol{\Sigma}_{i}^{-1} \frac{\partial \boldsymbol{\Sigma}_{i}}{\partial \tau} \boldsymbol{\Sigma}_{i}^{-1} \frac{\partial \boldsymbol{\Sigma}_{i}}{\partial \tau}=\frac{1}{\left(m_{i} \tau+\sigma^{2}\right)^{2}} m_{i} \mathbf{J}_{m_{i}}
$$

o que acarreta

$$
\operatorname{tr}\left(\boldsymbol{\Sigma}_{i}^{-1} \frac{\partial \boldsymbol{\Sigma}_{i}}{\partial \tau} \boldsymbol{\Sigma}_{i}^{-1} \frac{\partial \boldsymbol{\Sigma}_{i}}{\partial \tau}\right)=\left(\frac{m_{i}}{m_{i} \tau+\sigma^{2}}\right)^{2}
$$

Substituindo esses resultados na expressão (3.3.6) obtemos a partição $K_{\tau \tau}$ dada por 


$$
\begin{aligned}
\mathrm{K}_{\tau \tau} & =\sum_{i=1}^{n}\left\{-\frac{\left(\frac{m_{i}}{m_{i} \tau+\sigma^{2}}\right)^{2}}{4}+\frac{f_{g_{i}}}{m_{i}\left(m_{i}+2\right)}\left[\left(\frac{m_{i}}{m_{i} \tau+\sigma^{2}}\right)^{2}+2\left(\frac{m_{i}}{m_{i} \tau+\sigma^{2}}\right)^{2}\right]\right\} \\
& =\sum_{i=1}^{n}\left\{-\frac{\left(\frac{m_{i}}{m_{i} \tau+\sigma^{2}}\right)^{2}}{4}+\frac{f_{g_{i}}}{m_{i}\left(m_{i}+2\right)}\left[3\left(\frac{m_{i}}{m_{i} \tau+\sigma^{2}}\right)^{2}\right]\right\} \\
& =\sum_{i=1}^{n}\left(\frac{m_{i}}{m_{i} \tau+\sigma^{2}}\right)^{2}\left[-\frac{1}{4}+\frac{3 f_{g_{i}}}{m_{i}\left(m_{i}+2\right)}\right]
\end{aligned}
$$

que, sob $\mathrm{H}_{0}: \tau=0$, assume a forma

$$
\mathrm{K}_{\tau \tau}^{0}=\sum_{i=1}^{n}\left(-\frac{m_{i}^{2}}{4 \sigma_{0}^{4}}+\frac{3 f_{g_{i}} m_{i}}{\sigma_{0}^{4}\left(m_{i}+2\right)}\right)
$$

Para obter a partição $\mathrm{K}_{\sigma^{2} \sigma^{2}}$ temos que

$$
c_{i \sigma^{2} \sigma^{2}}=\operatorname{tr}\left(\boldsymbol{\Sigma}_{i}^{-1}\right) \operatorname{tr}\left(\boldsymbol{\Sigma}_{i}^{-1}\right)=\left[\frac{m_{i}}{\sigma^{2}}\left(\frac{\left(m_{i}-1\right) \tau+\sigma^{2}}{m_{i} \tau+\sigma^{2}}\right)\right]^{2}
$$

e,

$$
\begin{aligned}
\boldsymbol{\Sigma}_{i}^{-1} \frac{\partial \boldsymbol{\Sigma}_{i}}{\partial \sigma^{2}} \boldsymbol{\Sigma}_{i}^{-1} \frac{\partial \boldsymbol{\Sigma}_{i}}{\partial \sigma^{2}}=\boldsymbol{\Sigma}_{i}^{-1} \boldsymbol{\Sigma}_{i}^{-1} & =\frac{1}{\left(\sigma^{2}\right)^{2}}\left[\mathbf{I}_{m_{i}}-\frac{\tau}{m_{i} \tau+\sigma^{2}} \mathbf{J}_{m_{i}}\right]\left[\mathbf{I}_{m_{i}}-\frac{\tau}{m_{i} \tau+\sigma^{2}} \mathbf{J}_{m_{i}}\right] \\
& =\frac{1}{\left(\sigma^{2}\right)^{2}}\left[\mathbf{I}_{m_{i}}-\frac{2 \tau}{m_{i} \tau+\sigma^{2}} \mathbf{J}_{m_{i}}+\left(\frac{\tau}{m_{i} \tau+\sigma^{2}}\right)^{2} m_{i} \mathbf{J}_{m_{i}}\right]
\end{aligned}
$$

o que acarreta 


$$
\begin{aligned}
\operatorname{tr}\left(\Sigma_{i}^{-1} \Sigma_{i}^{-1}\right) & =\frac{1}{\left(\sigma^{2}\right)^{2}}\left[m_{i}-\frac{2 m_{i} \tau}{m_{i} \tau+\sigma^{2}}+\left(\frac{m_{i} \tau}{m_{i} \tau+\sigma^{2}}\right)^{2}\right] \\
& =\frac{1}{\left(\sigma^{2}\right)^{2}}\left[\left(m_{i}-1\right)+\left(1-\frac{m_{i} \tau}{m_{i} \tau+\sigma^{2}}\right)^{2}\right] \\
& =\frac{1}{\left(\sigma^{2}\right)^{2}}\left[\left(m_{i}-1\right)+\left(\frac{\sigma^{2}}{m_{i} \tau+\sigma^{2}}\right)^{2}\right] \\
& =\frac{\left(m_{i}-1\right)}{\sigma^{4}}+\frac{1}{\left(m_{i} \tau+\sigma^{2}\right)^{2}} \\
& =\frac{\left(m_{i}-1\right)\left(m_{i} \tau+\sigma^{2}\right)^{2}+\sigma^{4}}{\sigma^{4}\left(m_{i} \tau+\sigma^{2}\right)^{2}}
\end{aligned}
$$

Assim, substituindo esses resultados na expressão (3.3.6) temos

$$
\begin{aligned}
\mathrm{K}_{\sigma^{2} \sigma^{2}}= & \sum_{i=1}^{n}\left\{-\frac{\left[\frac{m_{i}}{\sigma^{2}}\left(\frac{\left(m_{i}-1\right) \tau+\sigma^{2}}{m_{i} \tau+\sigma^{2}}\right)\right]^{2}}{4}+\frac{\left[\frac{m_{i}}{\sigma^{2}}\left(\frac{\left(m_{i}-1\right) \tau+\sigma^{2}}{m_{i} \tau+\sigma^{2}}\right)\right]^{2} f_{g_{i}}}{m_{i}\left(m_{i}+2\right)}\right. \\
& \left.+\frac{2 f_{g_{i}}}{m_{i}\left(m_{i}+2\right)} \frac{\left(m_{i}-1\right)\left(m_{i} \tau+\sigma^{2}\right)^{2}+\sigma^{4}}{\sigma^{4}\left(m_{i} \tau+\sigma^{2}\right)^{2}}\right\},
\end{aligned}
$$

que, sob $\mathrm{H}_{0}: \tau=0$, assume a forma 


$$
\begin{aligned}
\mathrm{K}_{\sigma^{2} \sigma^{2}}^{0} & =\sum_{i=1}^{n}-\frac{\left[\frac{m_{i}}{\sigma_{0}^{2}}\right]^{2}}{4}+\frac{\left[\frac{m_{i}}{\sigma_{0}^{2}}\right]^{2} f_{g_{i}}}{m_{i}\left(m_{i}+2\right)}+\frac{2 f_{g_{i}}}{m_{i}\left(m_{i}+2\right)} \frac{\left(m_{i}-1\right) \sigma_{0}^{4}+\sigma_{0}^{4}}{\sigma_{0}^{4} \sigma_{0}^{4}} \\
& =\sum_{i=1}^{n}-\frac{m_{i}^{2}}{4 \sigma_{0}^{4}}+\frac{m_{i} f_{g_{i}}}{\sigma_{0}^{4}\left(m_{i}+2\right)}+\frac{2 f_{g_{i}}}{\sigma_{0}^{4}\left(m_{i}+2\right)} \\
& =\sum_{i=1}^{n}-\frac{m_{i}^{2}}{4 \sigma_{0}^{4}}+\frac{\left(m_{i}+2\right) f_{g_{i}}}{\sigma_{0}^{4}\left(m_{i}+2\right)} \\
& =\sum_{i=1}^{n}-\frac{m_{i}^{2}}{4 \sigma_{0}^{4}}+\frac{f_{g_{i}}}{\sigma_{0}^{4}}
\end{aligned}
$$

Por fim, para obter a partição $\mathrm{K}_{\sigma^{2} \tau}$, considere

$$
\begin{aligned}
c_{i \sigma^{2} \tau} & =\operatorname{tr}\left(\Sigma_{i}^{-1} \frac{\partial \Sigma_{i}}{\partial \sigma^{2}}\right) \operatorname{tr}\left(\Sigma_{i}^{-1} \frac{\partial \Sigma_{i}}{\partial \tau}\right) \\
& =\operatorname{tr}\left(\Sigma_{i}^{-1}\right)\left(\frac{m_{i}}{m_{i} \tau+\sigma^{2}}\right) \\
& =\frac{m_{i}}{\sigma^{2}}\left(\frac{\left(m_{i}-1\right) \tau+\sigma^{2}}{m_{i} \tau+\sigma^{2}}\right)\left(\frac{m_{i}}{m_{i} \tau+\sigma^{2}}\right) \\
& =\left(\frac{m_{i}}{m_{i} \tau+\sigma^{2}}\right)^{2} \frac{\left(m_{i}-1\right) \tau+\sigma^{2}}{\sigma^{2}}
\end{aligned}
$$

e. 


$$
\begin{aligned}
\left(\Sigma_{i}^{-1} \frac{\partial \boldsymbol{\Sigma}_{i}}{\partial \sigma^{2}}\right)\left(\Sigma_{i}^{-1} \frac{\partial \boldsymbol{\Sigma}_{i}}{\partial \tau}\right) & =\boldsymbol{\Sigma}_{i}^{-1}\left(\frac{1}{m_{i} \tau+\sigma^{2}} \mathbf{J}_{m_{i}}\right) \\
& =\frac{1}{\sigma^{2}}\left(\mathbf{I}_{m_{i}}-\frac{\tau}{m_{i} \tau+\sigma^{2}} \mathbf{J}_{m_{i}}\right) \mathbf{J}_{m_{i}} \frac{1}{m_{i} \tau+\sigma^{2}} \\
& =\frac{1}{\sigma^{2}\left(m_{i} \tau+\sigma^{2}\right)}\left(\mathbf{J}_{m_{i}}-\frac{m_{i} \tau}{m_{i} \tau+\sigma^{2}} \mathbf{J}_{m_{i}}\right) \\
& =\frac{1}{\sigma^{2}\left(m_{i} \tau+\sigma^{2}\right)}\left(\mathbf{I}_{m_{i}}-\frac{m_{i} \tau}{m_{i} \tau+\sigma^{2}} \mathbf{I}_{m_{i}}\right) \mathbf{J}_{m_{i}} \\
& =\frac{1}{\sigma^{2}\left(m_{i} \tau+\sigma^{2}\right)}\left(\frac{\sigma^{2}}{m_{i} \tau+\sigma^{2}}\right) \mathbf{J}_{m_{i}} \\
& =\left(\frac{1}{m_{i} \tau+\sigma^{2}}\right)^{2} \mathbf{J}_{m_{i}},
\end{aligned}
$$

o que acarreta

$$
\operatorname{tr}\left[\left(\boldsymbol{\Sigma}_{i}^{-1} \frac{\partial \boldsymbol{\Sigma}_{i}}{\partial \sigma^{2}}\right)\left(\boldsymbol{\Sigma}_{i}^{-1} \frac{\partial \boldsymbol{\Sigma}_{i}}{\partial \tau}\right)\right]=\frac{m_{i}}{\left(m_{i} \tau+\sigma^{2}\right)^{2}}
$$

Assim, substituindo esses resultados na expressão (3.3.6) temos

$$
\begin{aligned}
\mathrm{K}_{\sigma^{2} \tau}= & \left\{\sum_{i=1}^{n}-\frac{\left(\frac{m_{i}}{m_{i} \tau+\sigma^{2}}\right)^{2} \frac{\left(m_{i}-1\right) \tau+\sigma^{2}}{\sigma^{2}}}{4}+\frac{f_{g_{\mathrm{i}}}}{m_{i}\left(m_{i}+2\right)}\left(\frac{m_{i}}{m_{i} \tau+\sigma^{2}}\right)^{2} \frac{\left(m_{i}-1\right) \tau+\sigma^{2}}{\sigma^{2}}\right. \\
& \left.+2 \frac{f_{g_{i}}}{m_{i}\left(m_{i}+2\right)} \frac{m_{i}}{\left(m_{i} \tau+\sigma^{2}\right)^{2}}\right\},
\end{aligned}
$$


que, sob $\mathrm{H}_{0}: \tau=0$, assume a forma

$$
\begin{aligned}
\mathrm{K}_{\sigma^{2} \tau}^{0} & =\sum_{i=1}^{n}-\frac{m_{i}^{2}}{4 \sigma_{0}^{4}}+\frac{f_{g_{i}}}{m_{i}\left(m_{i}+2\right)} \frac{m_{i}^{2}}{\sigma_{0}^{4}}+2 \frac{f_{g_{i}}}{m_{i}\left(m_{i}+2\right)} \frac{m_{i}}{\sigma_{0}^{4}} \\
& =\sum_{i=1}^{n}-\frac{m_{i}^{2}}{4 \sigma_{0}^{4}}+\frac{f_{g_{i}} m_{i}\left(m_{i}+2\right)}{\sigma_{0}^{4} m_{i}\left(m_{i}+2\right)} \\
& =\sum_{i=1}^{n}-\frac{m_{i}^{2}}{4 \sigma_{0}^{4}}+\frac{f_{g_{i}}}{\sigma_{0}^{4}} .
\end{aligned}
$$

Utilizando resultados de álgebra matricial, temos que a inversa da matriz $\left[\begin{array}{cc}\mathrm{K}_{\sigma^{2} \sigma^{2}} & \mathrm{~K}_{\tau \sigma^{2}} \\ \mathrm{~K}_{\sigma^{2} \tau} & \mathrm{K}_{\tau \tau}\end{array}\right]$, que será denotada por

$$
\left[\begin{array}{cc}
\mathrm{K}^{\sigma^{2} \sigma^{2}} & \mathrm{~K}^{\tau \sigma^{2}} \\
\mathrm{~K}^{\sigma^{2} \tau} & \mathrm{K}^{\tau \tau}
\end{array}\right]
$$

é tal que

$$
\begin{aligned}
\mathrm{K}^{\tau \tau} & =\left[\mathrm{K}_{\tau \tau}-\mathrm{K}_{\tau \sigma^{2}} \mathrm{~K}_{\sigma^{2} \sigma^{2}}^{-1} \mathrm{~K}_{\sigma^{2} \tau}\right]^{-1}, \\
\mathrm{~K}^{\sigma^{2} \sigma^{2}} & =\mathrm{K}_{\sigma^{2} \sigma^{2}}^{-1}+\mathrm{K}_{\sigma^{2} \sigma^{2}}^{-1} \mathrm{~K}_{\sigma^{2} \tau}[\underbrace{\mathrm{K}_{\tau \tau}-\mathrm{K}_{\tau \sigma^{2}} \mathrm{~K}_{\sigma^{2} \sigma^{2}}^{-1} \mathrm{~K}_{\sigma^{2} \tau}}_{\mathrm{K}^{\tau \tau}}]^{-1} \mathrm{~K}_{\tau \sigma^{2}} \mathrm{~K}_{\sigma^{2} \sigma^{2}}^{-1}, \\
\mathrm{~K}^{\sigma^{2} \tau} & =-\mathrm{K}_{\sigma^{2} \sigma^{2}}^{-1} \mathrm{~K}_{\sigma^{2} \tau}[\underbrace{\mathrm{K}_{\tau \tau}-\mathrm{K}_{\tau \sigma^{2}} \mathrm{~K}_{\sigma^{2} \sigma^{2}}^{-1} \mathrm{~K}_{\sigma^{2} \tau}}_{\mathrm{K}^{\tau \tau}}]^{-1} \mathrm{e} \\
\mathrm{K}^{\tau \sigma^{2}} & =-[\underbrace{\mathrm{K}_{\tau \tau}-\mathrm{K}_{\tau \sigma^{2}} \mathrm{~K}_{\sigma^{2} \sigma^{2}}^{-1} \mathrm{~K}_{\sigma^{2} \tau}}_{\mathrm{K}^{\tau \tau}}]^{-1} \mathrm{~K}_{\tau \sigma^{2} \mathrm{~K}_{\sigma^{2} \sigma^{2}}^{-1}}
\end{aligned}
$$


Observando as expressões (3.3.7), (3.3.8) e (3.3.9) concluímos que, sob $\mathrm{H}_{0}$, $\mathrm{K}_{\sigma^{2} \sigma^{2}}=\mathrm{K}_{\sigma^{2} \tau}$, e, portanto segue que

$$
\begin{aligned}
\mathrm{K}^{\tau \tau 0} & =\left[\mathrm{K}_{\tau \tau}^{0}-\mathrm{K}_{\tau \sigma^{2}}^{0}\right]^{-1} \\
& =\left[\sum_{i=1}^{n}\left(-\frac{m_{i}^{2}}{4 \sigma_{0}^{4}}+\frac{3 f_{g_{i}} m_{i}}{\sigma_{0}^{4}\left(m_{i}+2\right)}\right)-\sum_{i=1}^{n}\left(-\frac{m_{i}^{2}}{4 \sigma_{0}^{4}}+\frac{f_{g_{i}}}{\sigma_{0}^{4}}\right)\right]^{-1} \\
& =\left[\sum_{i=1}^{n}-\frac{m_{i}^{2}}{4 \sigma_{0}^{4}}+\frac{m_{i}^{2}}{4 \sigma_{0}^{4}}+\frac{3 f_{g_{i}} m_{i}}{\sigma_{0}^{4}\left(m_{i}+2\right)}-\frac{f_{g_{i}}}{\sigma_{0}^{4}}\right)^{-1} \\
& =\left[\sum_{i=1}^{n} \frac{f_{g_{i}}}{\sigma_{0}^{4}}\left(\frac{3 m_{i}}{\left(m_{i}+2\right)}-1\right)\right]^{-1} \\
& =\left[\sum_{i=1}^{n} \frac{f_{g_{i}}}{\sigma_{0}^{4}}\left(\frac{3 m_{i}-m_{i}-2}{m_{i}+2}\right)\right]^{-1}=\left[\sum_{i=1}^{n} \frac{f_{g_{i}}}{\sigma_{0}^{4}} \frac{2\left(m_{i}-1\right)}{\left(m_{i}+2\right)}\right]^{-1} .
\end{aligned}
$$

Para desenvolver a expressão da estatística do tipo escore, é necessário utilizar a função escore de $\tau$ transformada, que obtemos utilizando os resultados (3.3.3) e $(3.3 .5)$ :

$$
\mathrm{S}_{\tau}^{* 0}=\left[\mathrm{S}_{\tau}^{0}-\mathrm{K}_{\tau \sigma^{2}}^{0}\left(\mathrm{~K}_{\sigma^{2} \sigma^{2}}^{0}\right)^{-1} \mathrm{~S}_{\sigma^{2}}^{0}\right]=\mathrm{S}_{\tau}^{0}-\mathrm{S}_{\sigma^{2}}^{0}
$$

pois, novamente, sob $\mathrm{H}_{0}$, temos $\mathrm{K}_{\sigma^{2} \sigma^{2}}=\mathrm{K}_{\sigma^{2} \tau}$. Assim, 


$$
\begin{aligned}
\mathrm{S}_{\tau}^{* 0} & =\sum_{i=1}^{n}\left\{-\frac{m_{i}}{2 \sigma_{0}^{2}}+\frac{v\left(u_{i}^{0}\right)}{2 \sigma_{0}^{4}}\left[\sum_{j=1}^{m_{i}}\left(y_{i j}-\mu_{i j}^{0}\right)\right]^{2}\right\}-\left\{\sum_{i=1}^{n}-\frac{m_{i}}{2 \sigma_{0}^{2}}+\sum_{i=1}^{n} \frac{v\left(u_{i}^{0}\right)}{2 \sigma_{0}^{4}}\left[\sum_{j=1}^{m_{i}}\left(y_{i j}-\mu_{i j}^{0}\right)^{2}\right]\right\} \\
& =\sum_{i=1}^{n} \frac{v\left(u_{i}^{0}\right)}{2 \sigma_{0}^{4}}\left[\sum_{j=1}^{m_{i}}\left(y_{i j}-\mu_{i j}^{0}\right)\right]^{2}-\sum_{i=1}^{n} \frac{v\left(u_{i}^{0}\right)}{2 \sigma_{0}^{4}}\left[\sum_{j=1}^{m_{i}}\left(y_{i j}-\mu_{i j}^{0}\right)^{2}\right] \\
& =\sum_{i=1}^{n} \frac{v\left(u_{i}^{0}\right)}{2 \sigma_{0}^{4}}\left\{\left[\sum_{j=1}^{m_{i}}\left(y_{i j}-\mu_{i j}^{0}\right)\right]^{2}-\sum_{j=1}^{m_{i}}\left(y_{i j}-\mu_{i j}^{0}\right)^{2}\right\} .
\end{aligned}
$$

Portanto a expressão da estatística do tipo escore $\mathrm{T}_{S}$, apresentada em (3.3.1) é obtida a partir de (3.3.7) e (3.3.10), e assume a forma expandida

$\mathrm{T}_{S}=\left\{\begin{array}{ll}\left\{\sum_{i=1}^{n} v\left(u_{i}^{0}\right)\left[\left(\sum_{j=1}^{m_{i}}\left(y_{i j}-\mu_{i j}^{0}\right)\right)^{2}-\sum_{j=1}^{m_{i}}\left(y_{i j}-\mu_{i j}^{0}\right)^{2}\right]\right\}^{2}\left[\sum_{i=1}^{n} \frac{f_{g_{i}}\left(m_{i}-1\right)}{8 \sigma_{0}^{4}\left(m_{i}+2\right)}\right]^{-1} & \text { se } \mathrm{S}_{\tau}^{* 0}>0 \\ 0 & \text { se } \mathrm{S}_{\tau}^{* 0} \leq 0\end{array}\right.$.

e, para grandes amostras, $\mathrm{T}_{S} \stackrel{\mathrm{H}_{0}}{\sim} \frac{1}{2} \chi_{0}^{2}+\frac{1}{2} \chi_{1}^{2}$.

Para o caso balanceado essa expressão se reduz a:

$$
\mathrm{T}_{S}=\left\{\begin{array}{ll}
\left\{\sum_{i=1}^{n} v\left(u_{i}^{0}\right)\left[\left(\sum_{j=1}^{m}\left(y_{i j}-\mu_{i j}^{0}\right)\right)^{2}-\sum_{j=1}^{m}\left(y_{i j}-\mu_{i j}^{0}\right)^{2}\right]\right\}^{2} \frac{8 \sigma_{0}^{4}(m+2)}{n f_{g_{i}}(m-1)} & \text { se } \mathrm{S}_{\tau}^{* 0}>0 \\
0 & \text { se } \mathrm{S}_{\tau}^{* 0} \leq 0
\end{array} .\right.
$$

\subsection{Teste para dois componentes de variância}

O modelo que inclui dois efeitos aleatórios independentes entre si, no intercepto e na inclinação, apresenta a formulação 


$$
\mathbf{y}_{i}=\mathbf{X}_{i} \beta+\mathbf{Z}_{i} \mathbf{b}_{i}+\epsilon_{i}, i=1, \ldots, n
$$

em que

$$
\mathbf{Z}_{i}=\left[\begin{array}{cc}
1 & z_{i 1} \\
1 & z_{i 2} \\
\vdots & \vdots \\
1 & z_{i m_{i}}
\end{array}\right]
$$

Para obter a matriz $\boldsymbol{\Sigma}_{i}=\mathbf{Z}_{i} \mathbf{D} \mathbf{Z}_{i}^{T}+\sigma^{2} \mathbf{I}_{m_{i}}$, vamos assumir $\mathbf{D}=\left[\begin{array}{cc}\tau_{1} & 0 \\ 0 & \tau_{2}\end{array}\right]$.

$$
\begin{aligned}
\mathbf{Z}_{i} \mathbf{D} \mathbf{Z}_{i}^{T} & =\left[\begin{array}{cc}
1 & z_{i 1} \\
1 & z_{i 2} \\
\vdots & \vdots \\
1 & z_{i m_{i}}
\end{array}\right]\left[\begin{array}{cc}
\tau_{1} & 0 \\
0 & \tau_{2}
\end{array}\right]\left[\begin{array}{ccc}
1 & \ldots & 1 \\
z_{i 1} & \ldots & z_{i m_{i}}
\end{array}\right] \\
& =\left[\begin{array}{cc}
\tau_{1} & \tau_{2} z_{i 1} \\
\tau_{1} & \tau_{2} z_{i 2} \\
\vdots & \vdots
\end{array}\right]\left[\begin{array}{ccc}
1 & \ldots & 1 \\
z_{i 1} & \ldots & z_{i m_{i}}
\end{array}\right] \\
& \\
\mathbf{Z}_{i} \mathbf{D} \mathbf{Z}_{i}^{T}= & \tau_{1} \mathbf{J}_{m_{i}}+\tau_{2}\left[\begin{array}{cccc}
z_{i 1}^{2} & z_{i 1} z_{i 2} & \ldots & z_{i 1} z_{i m_{i}} \\
z_{i 2} z_{i 1} & z_{i 2}^{2} & \ldots & z_{i 2} z_{i m_{i}} \\
\vdots & \vdots & & \vdots \\
z_{i m_{i}} z_{i 1} & z_{i m_{i}} z_{i 2} & \ldots & z_{i m_{i}}^{2}
\end{array}\right] \\
&
\end{aligned}
$$

o que acarreta $\boldsymbol{\Sigma}_{i}=\tau_{1} \mathbf{J}_{m_{i}}+\tau_{2} \mathbf{z}_{i} \mathbf{z}_{i}^{T}+\sigma^{2} \mathbf{I}_{m_{i}}$

Um possível interesse poderia ser testar $\mathrm{H}_{0}: \tau_{1}=\tau_{2}=0$ contra $\mathrm{H}_{1}: \tau_{1}>0$ e/ou $\tau_{2}>0$, ou seja, sob $\mathrm{H}_{0} \boldsymbol{\Sigma}_{i}=\sigma^{2} \mathbf{I}_{m_{i}}$. As expressões da função escore e informação de Fisher são obtidas, substituindo as quantidades 


$$
\frac{\partial \boldsymbol{\Sigma}_{i}}{\partial \sigma^{2}}=\mathbf{I}_{m_{i}}, \quad \frac{\partial \boldsymbol{\Sigma}_{i}}{\partial \tau_{1}}=\mathbf{J}_{m_{i}} \quad \text { e } \quad \frac{\partial \boldsymbol{\Sigma}_{i}}{\partial \tau_{2}}=\mathbf{z}_{i} \mathbf{z}_{i}^{T}
$$

nas expressões gerais apresentadas na Seção 2.2 .

Nesse caso $\boldsymbol{\tau}=\left(\tau_{1}, \tau_{2}\right)^{T}$ e $\boldsymbol{\lambda}=\left(\boldsymbol{\beta}^{T}, \sigma^{2}\right)^{T}$ e as partições da função escore são dadas por

$$
\mathrm{S}=\left(\mathrm{S}_{\lambda}, \mathrm{S}_{\tau}\right)^{T} \operatorname{com} \mathrm{S}_{\tau}=\left(\mathrm{S}_{\tau_{1}}, \mathrm{~S}_{\tau_{2}}\right)^{T} \text { e } \mathrm{S}_{\lambda}=\left(\mathrm{S}_{\beta}, \mathrm{S}_{\sigma^{2}}\right)^{T}
$$

Para obter as partições da matriz de informação de Fisher, considere

$$
\mathbf{K}=\left[\begin{array}{cccc}
\mathbf{K}_{\beta \beta} & \mathbf{K}_{\beta \sigma^{2}} & \mathbf{K}_{\beta \tau_{1}} & \mathbf{K}_{\beta \tau_{2}} \\
\mathbf{K}_{\sigma^{2} \beta} & \mathrm{K}_{\sigma^{2} \sigma^{2}} & \mathrm{~K}_{\sigma^{2} \tau_{1}} & \mathrm{~K}_{\sigma^{2} \tau_{2}} \\
\mathbf{K}_{\tau_{1} \beta} & \mathrm{K}_{\tau_{1} \sigma^{2}} & \mathrm{~K}_{\tau_{1} \tau_{1}} & \mathbf{K}_{\tau_{1} \tau_{2}} \\
\mathbf{K}_{\tau_{2} \beta} & \mathrm{K}_{\tau_{2} \sigma^{2}} & \mathrm{~K}_{\tau_{2} \tau_{1}} & \mathrm{~K}_{\tau_{2} \tau_{2}}
\end{array}\right]=\left[\begin{array}{cccc}
\mathbf{K}_{\beta \beta} & \mathbf{0} & \mathbf{0} & \mathbf{0} \\
0 & \mathrm{~K}_{\sigma^{2} \sigma^{2}} & \mathrm{~K}_{\sigma^{2} \tau_{1}} & \mathrm{~K}_{\sigma^{2} \tau_{2}} \\
\mathbf{0} & \mathrm{K}_{\tau_{1} \sigma^{2}} & \mathrm{~K}_{\tau_{1} \tau_{1}} & \mathrm{~K}_{\tau_{1} \tau_{2}} \\
\mathbf{0} & \mathrm{K}_{\tau_{2} \sigma^{2}} & \mathbf{K}_{\tau_{2} \tau_{1}} & \mathbf{K}_{\tau_{2} \tau_{2}}
\end{array}\right]=\left[\begin{array}{cc}
\mathbf{K}_{\lambda \lambda} & \mathbf{K}_{\lambda \tau} \\
\mathbf{K}_{\tau \lambda} & \mathbf{K}_{\tau \tau}
\end{array}\right]
$$

em que

$$
\begin{gathered}
\mathbf{K}_{\lambda \lambda}=\left[\begin{array}{cc}
\mathbf{K}_{\beta \beta} & 0 \\
0 & \mathrm{~K}_{\sigma^{2} \sigma^{2}}
\end{array}\right], \quad \mathbf{K}_{\tau \tau}=\left[\begin{array}{ll}
\mathrm{K}_{\tau_{1} \tau_{1}} & \mathrm{~K}_{\tau_{1} \tau_{2}} \\
\mathrm{~K}_{\tau_{2} \tau_{1}} & \mathrm{~K}_{\tau_{2} \tau_{2}}
\end{array}\right], \\
\mathbf{K}_{\lambda \tau}=\left[\begin{array}{cc}
0 & 0 \\
\mathrm{~K}_{\sigma^{2} \tau_{1}} & \mathrm{~K}_{\sigma^{2} \tau_{2}}
\end{array}\right] \text { e } \quad \mathbf{K}_{\tau \lambda}=\left[\begin{array}{ll}
0 & \mathrm{~K}_{\tau_{1} \sigma^{2}} \\
0 & \mathrm{~K}_{\tau_{2} \sigma^{2}}
\end{array}\right] .
\end{gathered}
$$

Nessas condições,

$$
\mathbf{K}^{\tau \tau}=\left(\mathbf{K}_{\tau \tau}-\mathbf{K}_{\tau \lambda} \mathbf{K}_{\lambda \lambda}^{-1} \mathbf{K}_{\lambda \tau}\right)^{-1},
$$




$$
\begin{aligned}
& \mathbf{K}_{\lambda \lambda}^{-1} \mathbf{K}_{\lambda \tau}=\left[\begin{array}{cc}
\mathbf{K}_{\beta \beta}^{-1} & 0 \\
0 & \mathrm{~K}_{\sigma^{2} \sigma^{2}}^{-1}
\end{array}\right]\left[\begin{array}{cc}
0 & \mathbf{0} \\
\mathrm{K}_{\sigma^{2} \tau_{1}} & \mathrm{~K}_{\sigma^{2} \tau_{2}}
\end{array}\right]=\left[\begin{array}{cc}
0 & 0 \\
\mathrm{~K}_{\sigma^{2} \sigma^{2}}^{-1} \mathrm{~K}_{\sigma^{2} \tau_{1}} & \mathrm{~K}_{\sigma^{2} \sigma^{2}}^{-1} \mathrm{~K}_{\sigma^{2} \tau_{2}}
\end{array}\right] \\
& \mathbf{K}_{\tau \lambda} \mathbf{K}_{\lambda \lambda}^{-1} \mathbf{K}_{\lambda \tau}=\left[\begin{array}{ll}
0 & \mathrm{~K}_{\tau_{1} \sigma^{2}} \\
0 & \mathrm{~K}_{\tau_{2} \sigma^{2}}
\end{array}\right]\left[\begin{array}{cc}
0 & 0 \\
\mathrm{~K}_{\sigma^{2} \sigma^{2}}^{-1} \mathrm{~K}_{\sigma^{2} \tau_{1}} & \mathrm{~K}_{\sigma^{2} \sigma^{2}}^{-1} \mathrm{~K}_{\sigma^{2} \tau_{2}}
\end{array}\right] \\
& =\left[\begin{array}{ll}
\mathrm{K}_{\tau_{1} \sigma^{2}} \mathrm{~K}_{\sigma^{2} \sigma^{2}}^{-1} \mathrm{~K}_{\sigma^{2} \tau_{1}} & \mathrm{~K}_{\tau_{1} \sigma^{2}} \mathrm{~K}_{\sigma^{2} \sigma^{2}}^{-1} \mathrm{~K}_{\sigma^{2} \tau_{2}} \\
\mathrm{~K}_{\tau_{2} \sigma^{2}} \mathrm{~K}_{\sigma^{2} \sigma^{2}}^{-1} \mathrm{~K}_{\sigma^{2} \tau_{1}} & \mathrm{~K}_{\tau_{2} \sigma^{2}} \mathrm{~K}_{\sigma^{2} \sigma^{2}}^{-1} \mathrm{~K}_{\sigma^{2} \tau_{2}}
\end{array}\right] \\
& \mathbf{K}^{\tau \tau}=\left[\begin{array}{ll}
\underbrace{}_{\tau_{\tau_{1} \tau_{1}}-\mathrm{K}_{\tau_{1} \sigma^{2}} \mathrm{~K}_{\sigma^{2} \sigma^{2}}^{-1} \mathrm{~K}_{\sigma^{2} \tau_{1}}} & \mathrm{~K}_{\tau_{1} \tau_{2}}-\mathrm{K}_{\tau_{1} \sigma^{2}} \mathrm{~K}_{\sigma^{2} \sigma^{2}}^{-1} \mathrm{~K}_{\sigma^{2} \tau_{2}} \\
\mathrm{~K}_{\tau_{1} \tau_{2}}-\mathrm{K}_{\tau_{2} \sigma^{2}} \mathrm{~K}_{\sigma^{2} \sigma^{2}}^{-1} \mathrm{~K}_{\sigma^{2} \tau_{1}} & \underbrace{\mathrm{K}_{\tau_{2} \tau_{2}}-\mathrm{K}_{\tau_{2} \sigma^{2}} \mathrm{~K}_{\sigma^{2} \sigma^{2}}^{-1} \mathrm{~K}_{\sigma^{2} \tau_{2}}}_{\operatorname{Var}\left(\tau_{2}\right)}
\end{array}\right] .
\end{aligned}
$$

Para obter a função escore transformada do vetor de interesse $\tau$, precisamos obter as partições

$$
\begin{gathered}
\mathbf{K}_{\lambda \lambda}^{-1}=\left[\begin{array}{cc}
\mathbf{K}_{\beta \beta}^{-1} & 0 \\
0 & \mathrm{~K}_{\sigma^{2} \sigma^{2}}^{-1}
\end{array}\right] \\
\mathbf{K}_{\tau \lambda}=\left[\begin{array}{ll}
0 & \mathrm{~K}_{\tau_{1} \sigma^{2}} \\
0 & \mathrm{~K}_{\tau_{2} \sigma^{2}}
\end{array}\right] \\
\mathbf{K}_{\tau \lambda} \mathbf{K}_{\lambda \lambda}^{-1}=\left[\begin{array}{ll}
0 & \mathrm{~K}_{\tau_{1} \sigma^{2}} \\
0 & \mathrm{~K}_{\tau_{2} \sigma^{2}}
\end{array}\right]\left[\begin{array}{cc}
\mathbf{K}_{\beta \beta}^{-1} & 0 \\
0 & \mathrm{~K}_{\sigma^{2} \sigma^{2}}^{-1}
\end{array}\right]=\left[\begin{array}{ll}
0 & \mathrm{~K}_{\tau_{1} \sigma^{2}} \mathrm{~K}_{\sigma^{\sigma^{2} \sigma^{2}}}^{-1} \\
0 & \mathrm{~K}_{\tau_{2} \sigma^{2}} \mathrm{~K}_{\sigma^{2} \sigma^{2}}^{-1}
\end{array}\right] .
\end{gathered}
$$

Para obter essas partições, devemos utilizar as expressões gerais apresentadas na Seção 2.2 e substituir em 


$$
\begin{aligned}
\mathrm{S}_{\tau}^{*} & =\left[\left(\begin{array}{l}
\mathrm{S}_{\tau_{1}} \\
\mathrm{~S}_{\tau_{2}}
\end{array}\right)-\left[\begin{array}{ll}
0 & \mathrm{~K}_{\tau_{1} \sigma^{2}} \mathrm{~K}_{\sigma^{2} \sigma^{2}}^{-1} \\
0 & \mathrm{~K}_{\tau_{2} \sigma^{2}} \mathrm{~K}_{\sigma^{2} \sigma^{2}}^{-1}
\end{array}\right]\left[\begin{array}{c}
\mathrm{S}_{\beta} \\
\mathrm{S}_{\sigma^{2}}
\end{array}\right]\right] \\
& =\left(\begin{array}{l}
\mathrm{S}_{\tau_{1}}-\mathrm{K}_{\tau_{1} \sigma^{2}} \mathrm{~K}_{\sigma^{2} \sigma^{2}}^{-1} \mathrm{~S}_{\sigma^{2}} \\
\mathrm{~S}_{\tau_{2}}-\mathrm{K}_{\tau_{2} \sigma^{2}} \mathrm{~K}_{\sigma^{2} \sigma^{2}}^{-1} \mathrm{~S}_{\sigma^{2}}
\end{array}\right),
\end{aligned}
$$

e a estatística do tipo escore para testar dois componentes de variância conjuntamente será dada por

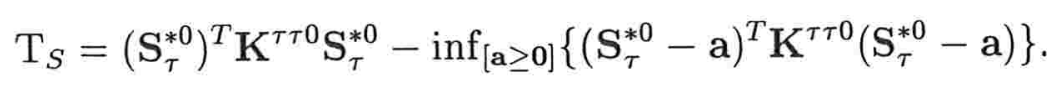

Para grandes amostras, como já foi visto anteriormente, $\mathrm{T}_{S} \stackrel{\mathrm{H}_{0}}{\sim} \omega_{0} \chi_{0}^{2}+\omega_{1} \chi_{1}^{2}+\omega_{2} \chi_{2}^{2}$, em que $\omega_{0}=\frac{1}{2 \pi} \cos ^{-1}(\rho), \omega_{1}=\frac{1}{2}$ e $\omega_{2}=\frac{1}{2}-\frac{1}{2 \pi} \cos ^{-1}(\rho)$, com $\rho$ sendo o coeficiente de correlação assintótico entre as estimativas de $\widehat{\tau}_{1}$ e $\widehat{\tau}_{2}$, (Kudô,1963). Como assumimos desde o início que a matriz $\mathbf{D}$ é diagonal, é razoável esperar que a correlação assintótica entre $\widehat{\tau}_{1}$ e $\widehat{\tau}_{2}$ seja próxima de zero, e, portanto, recaímos na distribuição $\frac{1}{4} \chi_{0}^{2}+\frac{1}{2} \chi_{1}^{2}+\frac{1}{4} \chi_{2}^{2}$.

\subsection{Teste para o componente de variância re- ferente ao efeito aleatório na inclinação na presença do efeito aleatório no intercepto}

Como já foi visto, no modelo que inclui dois efeitos aleatórios independentes entre si, no intercepto e na inclinação, a matriz de variâncias-covariâncias assume a forma $\Sigma_{i}=\tau_{1} \mathbf{J}_{m_{i}}+\tau_{2} \mathbf{Z}_{i} \mathbf{z}_{i}^{T}+\sigma^{2} \mathbf{I}_{m_{i}}$. Podemos estar interessados em testar $\mathrm{H}_{0}: \tau_{2}=0$ contra $\mathrm{H}_{1}: \tau_{2}>0$ com $\tau_{1}$ presente no modelo, ou seja, sob $\mathrm{H}_{0}$ temos $\Sigma_{i}^{0}=\tau_{1}^{0} \mathbf{J}_{m_{i}}+\sigma_{0}^{2} \mathbf{I}_{m_{i}}$. Da mesma forma que no caso anterior, as expressões da função escore e informação de Fisher são obtidas, substituindo as quantidades 


$$
\frac{\partial \boldsymbol{\Sigma}_{i}}{\partial \sigma^{2}}=\mathbf{I}_{m_{i}}, \quad \frac{\partial \boldsymbol{\Sigma}_{i}}{\partial \tau_{1}}=\mathbf{J}_{m_{i}} \quad \text { e } \quad \frac{\partial \boldsymbol{\Sigma}_{i}}{\partial \tau_{2}}=\mathbf{z}_{i} \mathbf{z}_{i}^{T},
$$

nas expressões gerais apresentadas na Seção 2.2 .

Nessa situação, como o interesse é desenvolver um teste somente sobre o componente de variância $\tau_{2}$, o vetor de perturbação é dado por $\boldsymbol{\lambda}^{T}=\left(\boldsymbol{\beta}^{T}, \sigma^{2}, \tau_{1}\right)^{T}$. Assim, as partições da função escore e da matriz de informação de Fisher serão dadas por

$$
\mathrm{S}_{\tau}=\mathrm{S}_{\tau_{2}} \quad \text { e } \quad \mathrm{S}_{\lambda}=\left(\mathrm{S}_{\beta}^{T}, \mathrm{~S}_{\sigma^{2}}, \mathrm{~S}_{\tau_{1}}\right)^{T},
$$

$\mathrm{e}$,

$\mathbf{K}=\left[\begin{array}{cccc}\mathbf{K}_{\beta \beta} & \mathbf{K}_{\beta \sigma^{2}} & \mathbf{K}_{\beta \tau_{1}} & \mathbf{K}_{\beta \tau_{2}} \\ \mathbf{K}_{\sigma^{2} \beta} & \mathrm{K}_{\sigma^{2} \sigma^{2}} & \mathrm{~K}_{\sigma^{2} \tau_{1}} & \mathrm{~K}_{\sigma^{2} \tau_{2}} \\ \mathbf{K}_{\tau_{1} \beta} & \mathrm{K}_{\tau_{1} \sigma^{2}} & \mathrm{~K}_{\tau_{1} \tau_{1}} & \mathrm{~K}_{\tau_{1} \tau_{2}} \\ \mathbf{K}_{\tau_{2} \beta} & \mathrm{K}_{\tau_{2} \sigma^{2}} & \mathrm{~K}_{\tau_{2} \tau_{1}} & \mathbf{K}_{\tau_{2} \tau_{2}}\end{array}\right]=\left[\begin{array}{cccc}\mathbf{K}_{\beta \beta} & \mathbf{0} & \mathbf{0} & \mathbf{0} \\ \mathbf{0} & \mathrm{K}_{\sigma^{2} \sigma^{2}} & \mathrm{~K}_{\sigma^{2} \tau_{1}} & \mathrm{~K}_{\sigma^{2} \tau_{2}} \\ \mathbf{0} & \mathrm{K}_{\tau_{1} \sigma^{2}} & \mathrm{~K}_{\tau_{1} \tau_{1}} & \mathrm{~K}_{\tau_{1} \tau_{2}} \\ \mathbf{0} & \mathrm{K}_{\tau_{2} \sigma^{2}} & \mathrm{~K}_{\tau_{2} \tau_{1}} & \mathrm{~K}_{\tau_{2} \tau_{2}}\end{array}\right]=\left[\begin{array}{cc}\mathbf{K}_{\lambda \lambda} & \mathbf{K}_{\lambda \tau} \\ \mathbf{K}_{\tau \lambda} & \mathbf{K}_{\tau \tau}\end{array}\right]$

em que

$$
\begin{aligned}
\mathbf{K}_{\lambda \lambda} & =\left[\begin{array}{ccc}
\mathbf{K}_{\beta \beta} & 0 & 0 \\
\mathbf{0} & \mathrm{K}_{\sigma^{2} \sigma^{2}} & \mathrm{~K}_{\sigma^{2} \tau_{1}} \\
\mathbf{0} & \mathrm{K}_{\tau_{1} \sigma^{2}} & \mathrm{~K}_{\tau_{1} \tau_{1}}
\end{array}\right], \quad \mathbf{K}_{\tau \tau}=\mathrm{K}_{\tau_{2} \tau_{2}}, \\
\mathbf{K}_{\tau \lambda} & =\left[\begin{array}{lll}
0 & \mathrm{~K}_{\tau_{2} \sigma^{2}} & \mathrm{~K}_{\tau_{2} \tau_{1}}
\end{array}\right] \text { e } \quad \mathbf{K}_{\lambda \tau}=\left[\begin{array}{c}
0 \\
\mathrm{~K}_{\sigma^{2} \tau_{2}} \\
\mathrm{~K}_{\tau_{1} \tau_{2}}
\end{array}\right] .
\end{aligned}
$$

Novamente,

$$
\mathbf{K}^{\tau \tau}=\left(\mathbf{K}_{\tau \tau}-\mathbf{K}_{\tau \lambda} \mathbf{K}_{\lambda \lambda}^{-1} \mathbf{K}_{\lambda \tau}\right)^{-1}
$$

Para obter essa expressão devemos utilizar resultados de álgebra matricial para calcular 


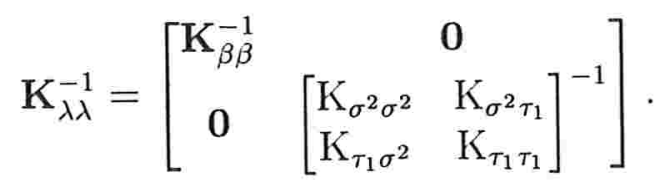

Para tanto, vamos usar o resultado

$$
\left[\begin{array}{cc}
\mathrm{K}_{\sigma^{2} \sigma^{2}} & \mathrm{~K}_{\sigma^{2} \tau_{1}} \\
\mathrm{~K}_{\tau_{1} \sigma^{2}} & \mathrm{~K}_{\tau_{1} \tau_{1}}
\end{array}\right]^{-1}=\left[\begin{array}{cc}
\mathrm{K}_{\sigma^{2} \sigma^{2}}-\mathrm{FE}^{-1} \mathrm{~F}^{T} & -\mathrm{FE}^{-1} \\
-\mathrm{E}^{-1} \mathrm{~F}^{T} & -\mathrm{E}^{-1}
\end{array}\right]=\left[\begin{array}{cc}
\mathrm{A} & \mathrm{B} \\
\mathrm{C} & \mathrm{D}
\end{array}\right],
$$

em que

$$
\begin{aligned}
& \mathrm{E}=\mathrm{K}_{\tau_{1} \tau_{1}}-\mathrm{K}_{\tau_{1} \sigma^{2}} \mathrm{~K}_{\sigma^{2} \sigma^{2}}^{-1} \mathrm{~K}_{\sigma^{2} \tau_{1}}, \\
& \mathrm{~F}=\mathrm{K}_{\sigma^{2} \sigma^{2}}^{-1} \mathrm{~K}_{\sigma^{2} \tau_{1}} .
\end{aligned}
$$

Dessa forma

$$
\begin{aligned}
& \mathbf{K}_{\lambda \lambda}^{-1}=\left[\begin{array}{ccc}
\mathbf{K}_{\beta \beta}^{-1} & \mathbf{0} & 0 \\
\mathbf{0} & \mathrm{A} & \mathrm{B} \\
\mathbf{0} & \mathrm{C} & \mathrm{D}
\end{array}\right] \\
& \mathbf{K}_{\tau \lambda} \mathbf{K}_{\lambda \lambda}^{-1}=\left[\begin{array}{lll}
\mathbf{0}^{T} & \mathrm{~K}_{\tau_{2} \sigma^{2}} & \mathrm{~K}_{\tau_{2} \tau_{1}}
\end{array}\right]\left[\begin{array}{ccc}
\mathbf{K}_{\beta \beta}^{-1} & \mathbf{0} & \mathbf{0} \\
\mathbf{0} & \mathrm{A} & \mathrm{B} \\
\mathbf{0} & \mathrm{C} & \mathrm{D}
\end{array}\right] \\
& =\left[\begin{array}{lll}
0^{T} & \left(\mathrm{~K}_{\tau_{2} \sigma^{2}} \mathrm{~A}+\mathrm{K}_{\tau_{2} \tau_{1}} \mathrm{C}\right) & \left(\mathrm{K}_{\tau_{2} \sigma^{2}} \mathrm{~B}+\mathrm{K}_{\tau_{2} \tau_{1}} \mathrm{D}\right)
\end{array}\right], \\
& \mathbf{K}_{\tau \lambda} \mathrm{K}_{\lambda \lambda}^{-1} \mathbf{K}_{\lambda \tau}=\left[\begin{array}{lll}
0^{T} & \left(\mathrm{~K}_{\tau_{2} \sigma^{2}} \mathrm{~A}+\mathrm{K}_{\tau_{2} \tau_{1}} \mathrm{C}\right) & \left(\mathrm{K}_{\tau_{2} \sigma^{2}} \mathrm{~B}+\mathrm{K}_{\tau_{2} \tau_{1}} \mathrm{D}\right)
\end{array}\right]\left[\begin{array}{c}
0 \\
\mathrm{~K}_{\sigma^{2} \tau_{2}} \\
\mathrm{~K}_{\tau_{1} \tau_{2}}
\end{array}\right] \\
& =\left(\mathrm{K}_{\tau_{2} \sigma^{2}} \mathrm{~A}+\mathrm{K}_{\tau_{2} \tau_{1}} \mathrm{C}\right) \mathrm{K}_{\sigma^{2} \tau_{2}}+\left(\mathrm{K}_{\tau_{2} \sigma^{2}} \mathrm{~B}+\mathrm{K}_{\tau_{2} \tau_{1}} \mathrm{D}\right) \mathrm{K}_{\tau_{1} \tau_{2}},
\end{aligned}
$$


e, portanto

$$
\mathrm{K}^{\tau \tau}=\left\{\mathrm{K}_{\tau_{2} \tau_{2}}-\left(\mathrm{K}_{\tau_{2} \sigma^{2}} \mathrm{~A}+\mathrm{K}_{\tau_{2} \tau_{1}} \mathrm{C}\right) \mathrm{K}_{\sigma^{2} \tau_{2}}-\left(\mathrm{K}_{\tau_{2} \sigma^{2}} \mathrm{~B}+\mathrm{K}_{\tau_{2} \tau_{1}} \mathrm{D}\right) \mathrm{K}_{\tau_{1} \tau_{2}}\right\}^{-1} .
$$

Para obter a função escore transformada de $\tau_{2}$ considere

$$
\begin{aligned}
& \mathbf{K}_{\tau \lambda} \mathbf{K}_{\lambda \lambda}^{-1} \mathbf{S}_{\lambda}=\left[\begin{array}{lll}
0^{T} & \left(\mathrm{~K}_{\tau_{2} \sigma^{2}} \mathrm{~A}+\mathrm{K}_{\tau_{2} \tau_{1}} \mathrm{C}\right) & \left(\mathrm{K}_{\tau_{2} \sigma^{2}} \mathrm{~B}+\mathrm{K}_{\tau_{2} \tau_{1}} \mathrm{D}\right)
\end{array}\right]\left[\begin{array}{c}
\mathrm{S}_{\beta} \\
\mathrm{S}_{\sigma^{2}} \\
\mathrm{~S}_{\tau_{1}}
\end{array}\right] \\
& =\left(K_{\tau_{2} \sigma^{2}} \mathrm{~A}+\mathrm{K}_{\tau_{2} \tau_{1}} \mathrm{C}\right) \mathrm{S}_{\sigma^{2}}+\left(\mathrm{K}_{\tau_{2} \sigma^{2}} \mathrm{~B}+\mathrm{K}_{\tau_{2} \tau_{1}} \mathrm{D}\right) \mathrm{S}_{\tau_{1}} \text {, }
\end{aligned}
$$

e,

$$
\mathrm{S}_{\tau}^{*}=\left\{\mathrm{S}_{\tau_{2}}-\left(\mathrm{K}_{\tau_{2} \sigma^{2}} \mathrm{~A}+\mathrm{K}_{\tau_{2} \tau_{1}} \mathrm{C}\right) \mathrm{S}_{\sigma^{2}}-\left(\mathrm{K}_{\tau_{2} \sigma^{2}} \mathrm{~B}+\mathrm{K}_{\tau_{2} \tau_{1}} \mathrm{D}\right) \mathrm{S}_{\tau_{1}}\right\}
$$

Assim, a estatística do tipo escore é obtida, substituindo-se as expressões acima em

$$
\mathrm{T}_{S}= \begin{cases}\left(\mathrm{S}_{\tau}^{* 0}\right)^{2} \mathrm{~K}^{\tau \tau 0} & \text { se } \mathrm{S}_{\tau}^{* 0} \geq 0 \\ 0 & \text { se } \mathrm{S}_{\tau}^{* 0}<0\end{cases}
$$

e, para grandes amostras, $\mathrm{T}_{S} \stackrel{\mathrm{H}_{0}}{\sim} \frac{1}{2} \chi_{0}^{2}+\frac{1}{2} \chi_{1}^{2}$. 


\section{Capítulo 4}

\section{Aplicações}

Foram desenvolvidas subrotinas na linguagem de programação Ox (ver, por exemplo, Doornik, 2001) para ajustar os modelos elípticos lineares mistos assumindo distribuições multivariadas t-Student e exponencial potência, que foram utilizadas nos exemplos que se seguem. Também para essas distribuições foram desenvolvidas subrotinas para calcular a estatística do tipo escore para várias situações de testes. Os programas estão disponíveis no endereço http://www. de ufpe . $\mathrm{br} / \sim$ cysneiros/ftp/SavalliPaulaCysneiros.zip e podem ser aplicados em dados balanceados ou desbalanceados.

\subsection{Dados de Potthoff-Roy}

Como já vimos pela análise descritiva apresentada na introdução para os dois grupos, meninos e meninas, a resposta parece crescer linearmente com a idade e ainda existe uma variação no intercepto dos perfis individuais. Como aparecem alguns perfis atípicos para o grupo de meninos vamos assumir inicialmente o modelo normal tradicional e comparar os resultados supondo, posteriormente, erros elípticos com caudas mais pesadas para o grupo de meninos.

Assumiremos para a resposta da $i$-ésima criança ("cluster") o seguinte modelo elíptico marginal:

$$
\mathrm{Y}_{i}=\mathrm{X}_{i} \boldsymbol{\beta}+\boldsymbol{\xi}_{i}, i=1, \ldots, 2 \bar{\tau},
$$


em que $\boldsymbol{\xi}_{i} \sim \mathrm{El}_{4}\left(\mathbf{0} ; \mathbf{Z}_{i} \mathbf{D} \mathbf{Z}_{i}^{T}+\sigma^{2} \mathbf{I}_{4}\right)$,

$$
\mathbf{X}_{i}=\left[\begin{array}{cccc}
1 & 8 & 0 & 0 \\
1 & 10 & 0 & 0 \\
1 & 12 & 0 & 0 \\
1 & 14 & 0 & 0
\end{array}\right]
$$

para as meninas, e,

$$
\mathbf{X}_{i}=\left[\begin{array}{cccc}
0 & 0 & 1 & 8 \\
0 & 0 & 1 & 10 \\
0 & 0 & 1 & 12 \\
0 & 0 & 1 & 14
\end{array}\right]
$$

para os meninos, de tal forma que $\beta=\left(\alpha_{g}, \beta_{g}, \alpha_{b}, \beta_{b}\right)^{T}$. Os parâmetros fixos foram indexados por $g$ para meninas ("girls") e $b$ para meninos ("boys"). Vamos assumir três possíveis estruturas para a matriz de variâncias-covariâncias :

(i) Modelo com efeito aleatório no intercepto: $\mathbf{Z}_{i}=\mathbf{1}_{\mathbf{4}}$ e $\mathbf{D}=\tau_{1}$;

(ii) Modelo com efeito aleatório na inclinação: $\mathbf{Z}_{i}=(8,10,12,14)^{T}$ e $\mathbf{D}=\tau_{2}$;

(iii) Modelo com 2 efeitos aleatórios, no intercepto e inclinação: $\mathbf{Z}_{i}=\left[\begin{array}{cccc}1 & 1 & 1 & 1 \\ 8 & 10 & 12 & 14\end{array}\right]^{T}$ e $\mathbf{D}=\operatorname{diag}\left[\tau_{1}, \tau_{2}\right]$.

Considerando a estatística tipo escore, o modelo de independência foi testado contra os modelos (i), (ii) e (iii). Uma outra situação foi considerada com o intuito de testar a necessidade de incluir um efeito aleatório na inclinação quando o efeito aleatório no intercepto já está no modelo. Os valores de $\mathrm{T}_{S}$ sob a suposição de normalidade para os erros estão apresentados na Tabela 4.1. Nas três primeiras situações, a hipótese nula de independência foi rejeitada. Na última situação, a hipótese nula não foi rejeitada a um nível de significância de 5\%, e, portanto, concluímos que o modelo final deve incluir somente o efeito aleatório no intercepto.

Uma análise de resíduos para o modelo normal final foi feita utilizando o resíduo condicional padronizado, e está apresentada na Figura 4.1. Observe que três 
observações foram identificadas com valores aberrantes. Duas dessas observações correspondem ao menino 9 nas idades de 10 e 12 anos, enquanto que a terceira corresponde ao menino 13 na idade de 8 anos. A influência ao retirar essas observações nas estimativas dos parâmetros, assim como na estatística $\mathrm{T}_{S}$, foi avaliada (Tabela 4.2) e as variações alcançam até $30 \%$.

Todas as análises foram repetidas assumindo agora uma distribuição t-Student multivariada com 6 graus de liberdade para o grupo dos meninos e 30 graus de liberdade para as meninas, como sugerido por Pinheiro, Liu e Wu (2001). Para a distribuição t-Student $\operatorname{com} \nu_{i}$ graus de liberdade encontramos:

$$
\begin{aligned}
v\left(u_{i}\right) & =\frac{\nu_{i}+m_{i}}{\nu_{i}+u_{i}}, \\
d_{g_{i}} & =\frac{\left(\nu_{i}+m_{i}\right) m_{i}}{4\left(\nu_{i}+m_{i}+2\right)} \mathrm{e} \\
f_{g_{i}} & =\frac{\left(\nu_{i}+m_{i}\right)\left(m_{i}+2\right) m_{i}}{4\left(\nu_{i}+m_{i}+2\right)} .
\end{aligned}
$$

Os resultados da estatística $\mathrm{T}_{S}$ para todas as hipóteses consideradas sob a suposição de distribuição t-Student estão também apresentadas na Tabela 4.1. Apesar de as conclusões não terem se alterado a um nível de significância de $5 \%$, e o modelo final escolhido sob a distribuição t-Student ter sido o mesmo que o escolhido sob a distribuição normal, podemos notar que a estatística $T_{S}$ assume valores maiores no modelo t-Student para todas as hipóteses testadas. Além disso, vale ressaltar que se tivéssemos adotado um nível de significância de $10 \%$, o modelo final sob a distribuição t-Student seria o modelo com intercepto e inclinação aleatórios.

As estimativas dos parâmetros do modelo com um efeito aleatório no intercepto para as duas distribuições estudadas estão apresentadas na Tabela 4.3. Podemos notar que as estimativas dos parâmetros fixos são similares para os dois 
Tabela 4.1: Resultados da estatística $\mathrm{T}_{S}$ para várias hipóteses envolvendo efeitos aleatórios sob a suposição de normalidade e distribuição t-Student em modelos lineares mistos ajustados para os dados de Potthoff-Roy.

\begin{tabular}{lrcrc}
\hline \hline Hipóteses & Normal & $\mathrm{p}$ & t-Student & $\mathrm{p}$ \\
\hline $\mathrm{H}_{0}: \tau_{1}=0$ & 61,8 & $<0,01^{1}$ & 62,5 & $<0,01^{1}$ \\
$\mathrm{H}_{1}: \tau_{1}>0$ & & & & \\
\hline $\mathrm{H}_{0}: \tau_{2}=0$ & 58,5 & $<0,01^{1}$ & 61,0 & $<0,01^{1}$ \\
$\mathrm{H}_{1}: \tau_{2}>0$ & & & & \\
\hline $\mathrm{H}_{0}: \tau_{1}=\tau_{2}=0$ & 62,2 & $<0,01^{2}$ & 63,5 & $<0,01^{2}$ \\
$\mathrm{H}_{1}: \tau_{1}>0$ e/ou $\tau_{2}>0$ & & & & \\
\hline $\mathrm{H}_{0}: \tau_{2}=0$ & 0,60 & $0,21^{1}$ & 1,95 & $0,08^{1}$ \\
$\mathrm{H}_{1}: \tau_{2}>0$ & & & & \\
com $\tau_{1}$ no modelo & & & & \\
\hline \hline
\end{tabular}

(1) Calculado com base na distribuição $\frac{1}{2} \chi_{0}^{2}+\frac{1}{2} \chi_{1}^{2}$.

(2) Calculado com base na distribuição $\frac{1}{4} \chi_{0}^{2}+\frac{1}{2} \chi_{1}^{2}+\frac{1}{4} \chi_{2}^{2}$.



Figura 4.1: Gráfico dos resíduos condicionais padronizados para o modelo com um efeito aleatório no intercepto assumindo distribuição normal para os dados de Potthoff-Roy. 
modelos, porém as estimativas dos erros-padrão são menores sob a suposição de distribuição t-Student multivariada. Isso significa que temos um modelo alternativo ao modelo normal, cujas estimativas são também consistentes e parecem ser mais precisas. Além disso, como veremos a seguir, as estimativas dos parâmetros mostram-se também robustas contra as observações aberrantes.

A influência da retirada das observações aberrantes que estão destacadas na Figura 4.1 nas estimativas dos parâmetros, assim como na estatística $T_{S}$, também foi avaliada sob o modelo t-Student (Tabela 4.2). As variações são, em geral, menores sob o modelo t-Student, confirmando a robustez desse modelo na presença de observações aberrantes, mesmo não tendo sido observadas mudanças nos resultados inferenciais.

A Figura 4.2 traz o gráfico dos resíduos condicionais padronizados no modelo t-Student multivariado. Os mesmos pontos identificados no modelo normal aparecem neste modelo, entretanto, de acordo com a Tabela 4.4, que apresenta os pesos $v\left(\hat{u}_{i}\right)$ 's de cada "cluster", essas observações referentes aos meninos 9 e 13 receberam pesos menores que os outros no processo de estimação do modelo t-Student multivariado.

Ambos os modelos, normal e t-Student, não apresentaram pontos de alavanca, como mostram os gráficos da Figura 4.3. As Figuras 4.4 e 4.5 mostram o comportamento dos resíduos e alavanca generalizada em função dos pesos $v_{i}$, respectivamente. Como já havia sido ressaltado, observações com menores pesos são as que apresentam maiores resíduos e observações com maiores pesos são as que exercem maior influência nos próprios valores preditos.

A Figura 4.6 descreve os perfis individuais ajustados no modelo com um efeito aleatório no intercepto sob a suposição de distribuição t-Student.

\subsection{Dados da altura do ramo da mandíbula}

Os dados da altura do ramo da mandíbula foram analisados sob o modelo normal e sob a distribuição exponencial potência multivariada. O parâmetro de 
Tabela 4.2: Variação percentual nas estimativas dos parâmetros e na estatística $\mathrm{T}_{S}$ para os modelos com um efeito aleatório no intercepto sob a suposição de distribuição normal e t-Student, ajustados para os dados de Potthoff-Roy, após excluir as observações aberrantes destacadas na Figura 4.1.

\begin{tabular}{llrrrr}
\hline \hline & & Normal & & t-Student & \\
\hline & & Excluindo & Excluindo & Excluindo & Excluindo \\
& $(9.2,13.1)$ & 9.3 & $(9.2,13.1)$ & 9.3 \\
\hline Meninos & $\alpha_{b}$ - intercepto & $6,5 \%$ & $0,8 \%$ & $1,4 \%$ & $0,2 \%$ \\
& $\beta_{b}$ - inclinação & $-10,1 \%$ & $-2,6 \%$ & $-1,4 \%$ & $-1,4 \%$ \\
\hline Meninas & $\alpha_{g}$ - intercepto & $0,0 \%$ & $0,0 \%$ & $0,0 \%$ & $0,0 \%$ \\
& $\beta_{g}$ - inclinação & $0,0 \%$ & $0,0 \%$ & $-0,1 \%$ & $-0,1 \%$ \\
\hline & $\sigma^{2}$ & $-33,7 \%$ & $-21,9 \%$ & $-10,8 \%$ & $-5,7 \%$ \\
& $\tau_{1}$ & $7,8 \%$ & $6,0 \%$ & $2,2 \%$ & $0,9 \%$ \\
& $\mathrm{~T}_{S}$ & $28,7 \%$ & $25,3 \%$ & $21,2 \%$ & $7,9 \%$ \\
\hline \hline
\end{tabular}

Tabela 4.3: Estimativas dos parâmetros para o modelo com um efeito aleatório no intercepto sob as distribuições normal e t-Student ajustados para os dados de Potthoff-Roy.

\begin{tabular}{llrr}
\hline \hline & & $\begin{array}{r}\text { Normal } \\
\text { Grupo }\end{array}$ & $\begin{array}{r}\text { t-Student } \\
\text { Estimativa }\end{array}$ \\
$\begin{array}{rlrr}\text { Estimativa } \\
\text { (erro-padrão-padrão) }\end{array}$ \\
\hline Meninos & $\alpha_{b}$ - intercepto & $16,34(0,96)$ & $16,93(0,84)$ \\
& $\beta_{b}$ - inclinação & $0,78(0,08)$ & $0,72(0,06)$ \\
\hline Meninas & $\alpha_{g}$ - intercepto & $17,37(1,16)$ & $17,43(0,95)$ \\
& $\beta_{g}$ - inclinação & $0,48(0,09)$ & $0,47(0,07)$ \\
\hline & $\sigma^{2}$ & $1,87(0,29)$ & $1,04(0,20)$ \\
& $\tau_{1}$ & $3,03(0,96)$ & $2,85(0,94)$ \\
\hline \hline
\end{tabular}




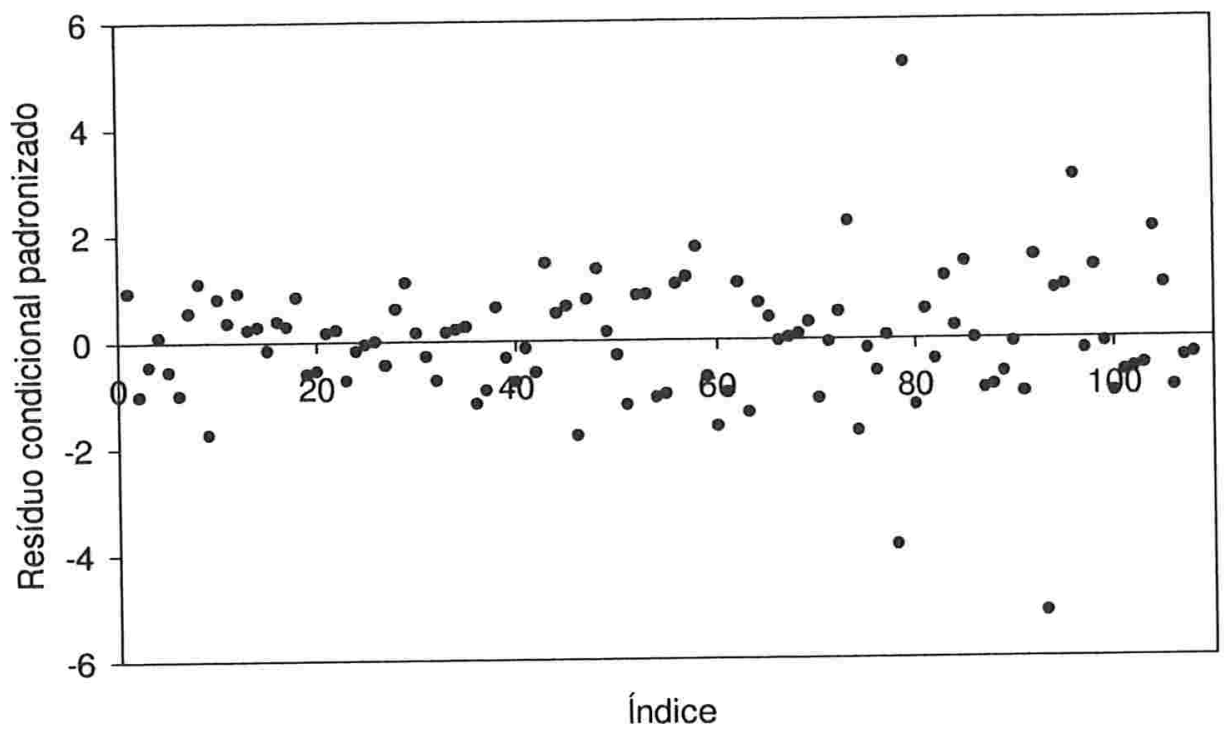

Figura 4.2: Gráfico dos resíduos condicionais padronizados para o modelo com um efeito aleatório no intercepto assumindo distribuição t-Student ajustado para os dados de Potthoff-Roy.

Tabela 4.4: Pesos estimados $v\left(\hat{u}_{i}\right)$ 's para o modelo t-Student com efeito aleatório no intercepto ajustado para os dados de Potthoff-Roy.

\begin{tabular}{cc|cc|cc|cc|cc}
\hline \hline Menino & $v\left(\hat{u}_{i}\right)$ & Menino & $v\left(\hat{u}_{i}\right)$ & Menino & $v\left(\hat{u}_{i}\right)$ & Menina & $v\left(\hat{u}_{i}\right)$ & Menina & $v\left(\hat{u}_{i}\right)$ \\
\hline 1 & 0,69 & 7 & 1,26 & $\mathbf{1 3}$ & $\mathbf{0 , 2 4}$ & 1 & 1,04 & 7 & 1,11 \\
2 & 1,13 & 8 & 0,70 & 14 & 1,13 & 2 & 1,04 & 8 & 1,06 \\
3 & 1,00 & $\mathbf{9}$ & $\mathbf{0 , 2 0}$ & 15 & 0,88 & 3 & 0,97 & 9 & 1,06 \\
4 & 0,70 & 10 & 0,70 & 16 & 1,11 & 4 & 1,07 & 10 & 0,92 \\
5 & 0,88 & 11 & 1,00 & & & 5 & 1,08 & 11 & 0,92 \\
6 & 1,45 & 12 & 1,01 & & & 6 & 1,09 & & \\
\hline \hline
\end{tabular}



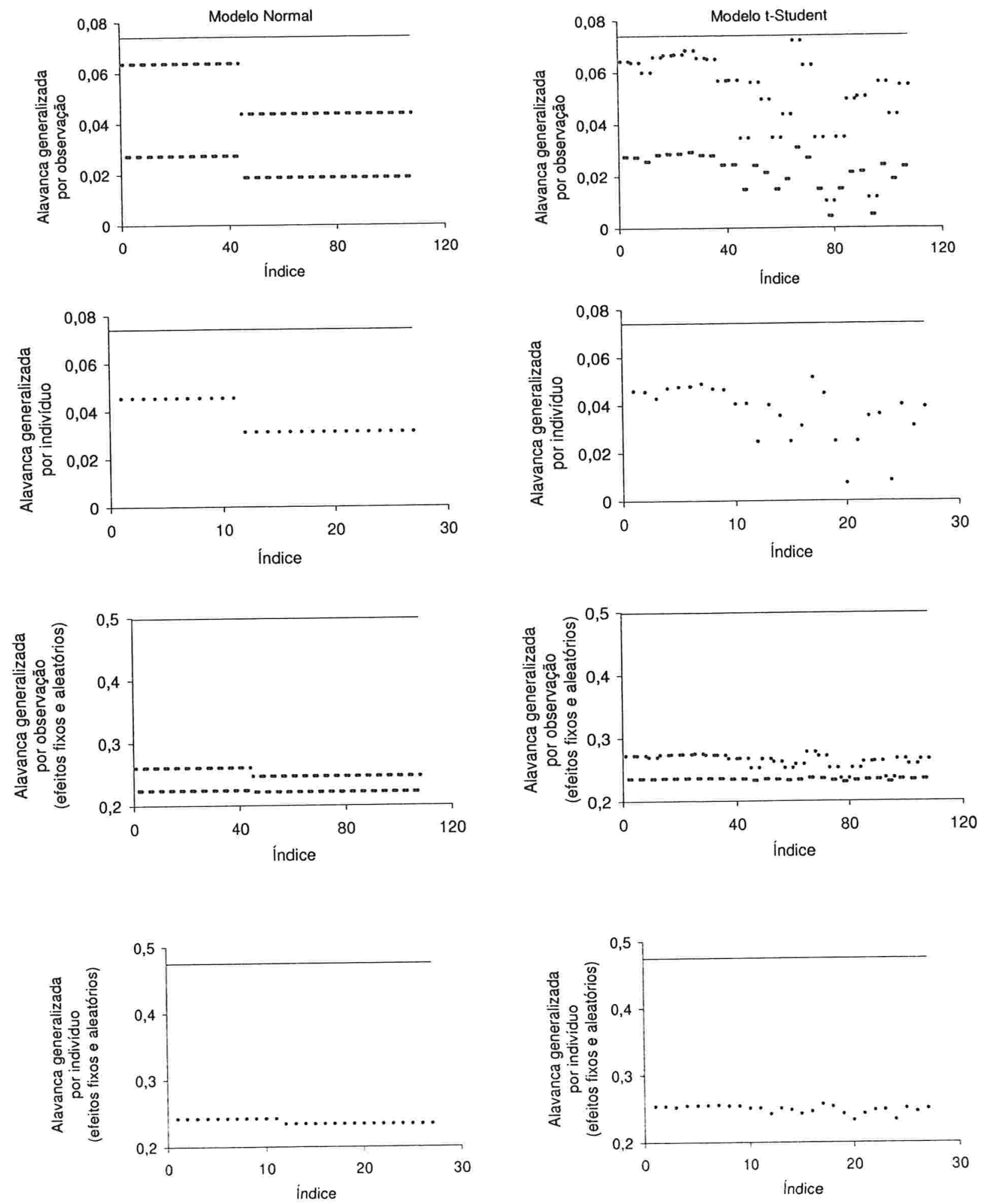

Figura 4.3: Gráficos da alavanca generalizada para o modelo com um efeito aleatório no intercepto assumindo distribuição t-Student ajustado para os dados de Potthoff-Roy. 


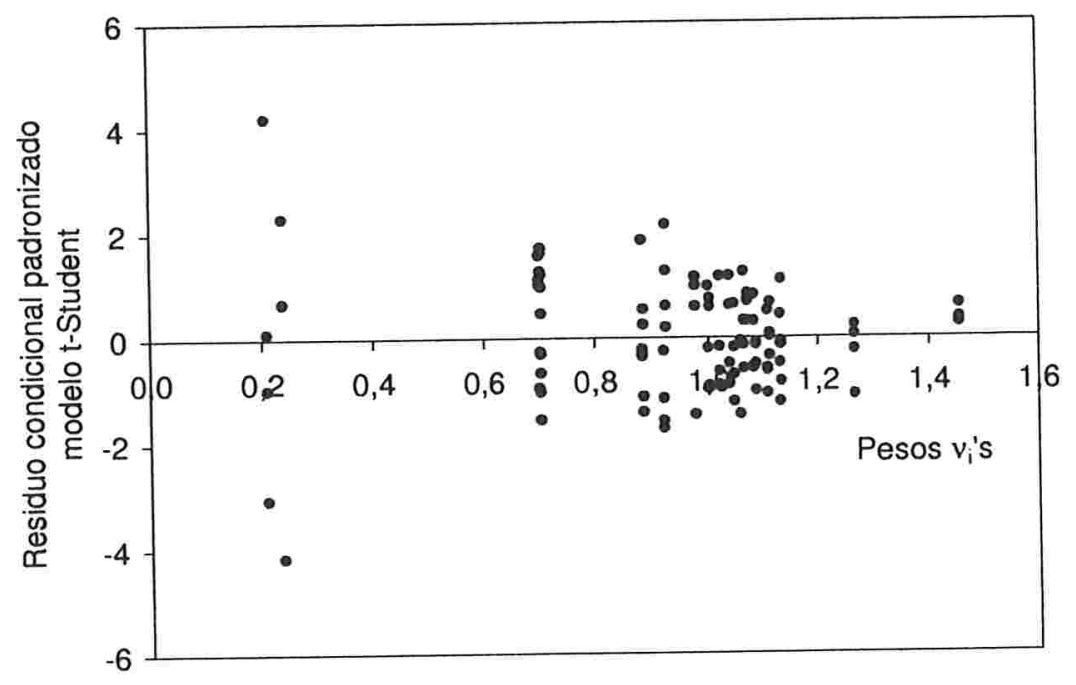

Figura 4.4: Gráfico dos pesos $v_{i}$ 's versus resíduo condicional padronizado para o modelo com um efeito aleatório no intercepto sob a suposição de distribuição t-Student para os dados de Potthoff-Roy.

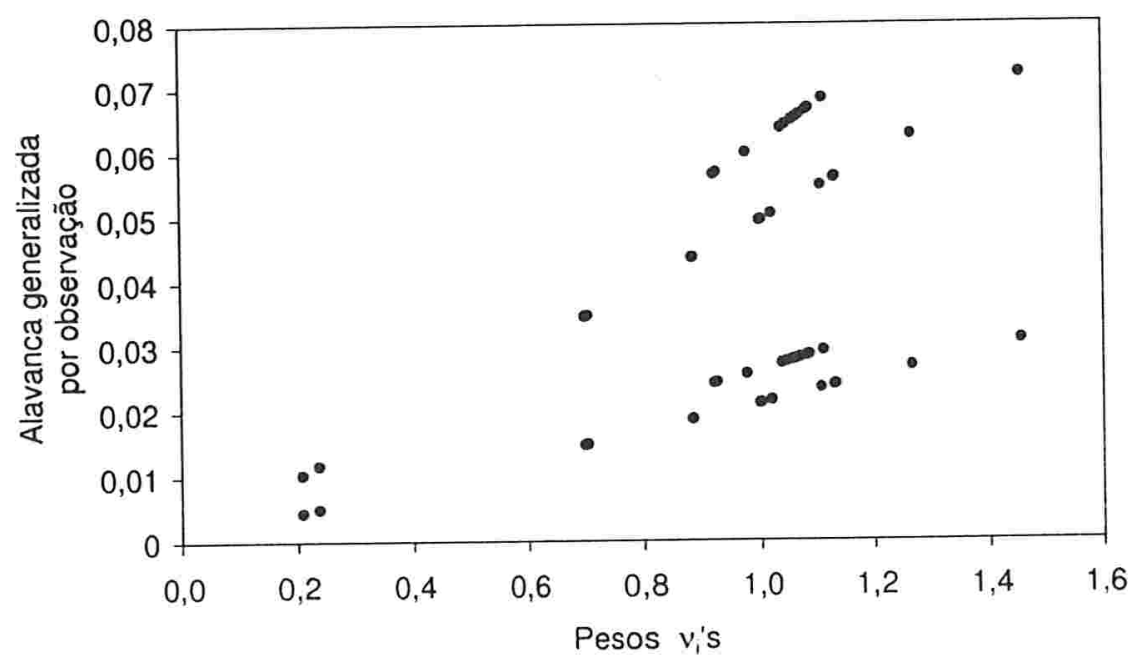

Figura 4.5: Gráfico dos pesos $v_{i}$ 's versus alavanca generalizada por observação para o modelo com um efeito aleatório no intercepto sob a suposição de distribuição t-Student para os dados de Potthoff-Roy. 


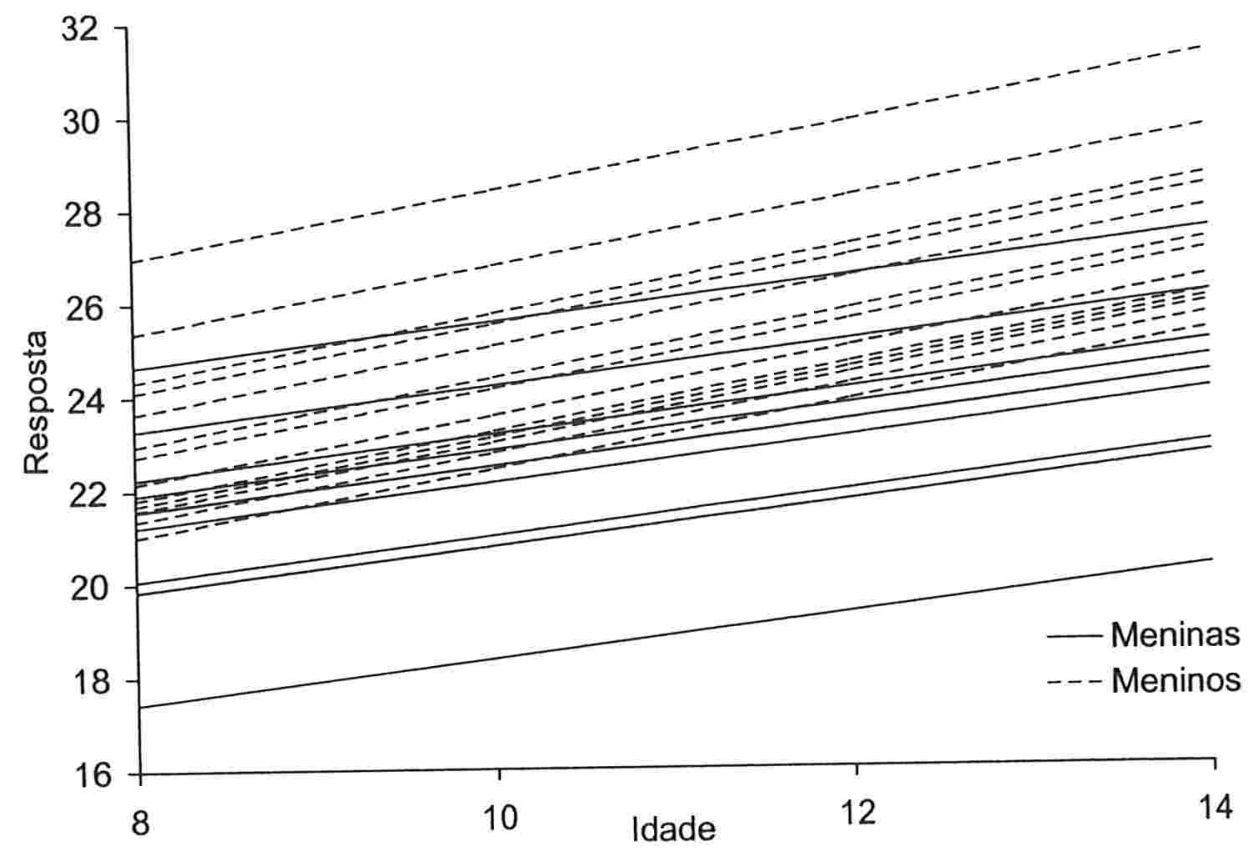

Figura 4.6: Perfis individuais ajustados para o modelo com um efeito aleatório no intercepto sob a suposição de distribuição t-Student para os dados de PotthoffRoy. 
forma da distribuição exponencial potência foi suposto o mesmo para todas as observações e foi escolhido a partir do critério da informação de Akaike no modelo de independência. O menor valor do AIC corresponde à $K=0,6$ como pode ser observado na Figura 4.7.

Para a distribuição exponencial potência com parâmetro de forma $K$ temos:

$$
\begin{aligned}
v\left(u_{i}\right) & =(1+K)^{-1} u_{i}^{\left[(1+K)^{-1}\right]-1}, \\
d_{g_{i}} & =\frac{1}{2^{-(1+K)}}(1+K)^{-2} \frac{\Gamma\left[\frac{m_{i}(1+K)+2-2 K}{2}\right]}{\Gamma\left[\frac{m_{i}(1+K)}{2}\right]} \mathrm{e} \\
f_{g_{i}} & =\frac{1}{4}(1+K)^{-2}\left[m_{i}(1+K)+2\right]\left[m_{i}(1+K)\right] .
\end{aligned}
$$

Iremos assumir para a resposta do $i$-ésimo menino o seguinte modelo marginal:

$$
\mathbf{y}_{i}=\mathbf{X}_{i} \boldsymbol{\beta}+\boldsymbol{\xi}_{i}, i=1, \ldots, 20
$$

em que $\boldsymbol{\xi}_{i} \sim \mathrm{PE}_{4}\left(\mathbf{0} ; \mathbf{Z}_{i} \mathbf{D} \mathbf{Z}_{i}^{T}+\sigma^{2} \mathbf{I}_{4}\right)$,

$$
\mathbf{X}_{i}=\left[\begin{array}{cc}
1 & 8,0 \\
1 & 8,5 \\
1 & 9,0 \\
1 & 9,5
\end{array}\right]
$$

e $\beta=(\alpha, \beta)^{T}$. Quatro estruturas para a matriz de variâncias-covariâncias foram consideradas:

(i) Modelo com um efeito aleatório no intercepto: $\mathbf{Z}_{i}=\mathbf{1}_{\mathbf{4}}$ e $\mathbf{D}=\tau_{1}$;

(ii) Modelo com um efeito aleatório na inclinação: $\mathbf{Z}_{i}=\left(\begin{array}{lllll}8,0 & 8,5 & 9,0 & 9,5\end{array}\right)^{T}$ e 




Figura 4.7: Critério da informação de Akaike para vários valores do parâmetro de forma $K$ da exponencial potência para os dados da altura do ramo da mandíbula.

$\mathbf{D}=\tau_{2}$

(iii) Modelo com 2 efeitos aleatórios, no intercepto e inclinação: $\mathbf{Z}_{i}=\left[\begin{array}{cccc}1 & 1 & 1 & 1 \\ 8,0 & 8,5 & 9,0 & 9,5\end{array}\right]^{T}$ e $\mathbf{D}=\operatorname{diag}\left[\tau_{1}, \tau_{2}\right]$

(iv) Modelo com 2 efeitos aleatórios, no intercepto e inclinação e com matriz não-diagonal $\mathbf{D}=\left[\begin{array}{cc}\tau_{1} & \tau_{12} \\ \tau_{12} & \tau_{2}\end{array}\right]$.

O modelo com matriz $\mathbf{D}$ não-diagonal foi considerado pois, ajustando retas individuais para cada menino calculamos o coeficiente de correlação entre o intercepto e a inclinação, que foi de -0.97 , indicando uma forte correlação entre esses dois efeitos.

Considerando a estatística do tipo escore, o modelo de independência foi testado contra o modelo com um efeito aleatório no intercepto $\left(\mathbf{D}=\tau_{1}\right)$ e contra o modelo com um efeito aleatório na inclinação $\left(\mathbf{D}=\tau_{2}\right)$. Duas outras situações foram propostas para testar a necessidade de incluir um efeito aleatório na inclina- 
ção quando o efeito aleatório no intercepto está no modelo, primeiro considerando a matriz $\mathbf{D}$ diagonal e, em seguida, considerando a matriz $\mathbf{D}$ não-diagonal.

Os valores da estatística do tipo escore $\mathrm{T}_{S}$ sob o modelo normal e exponencial potência estão apresentados na Tabela 4.5. Os mesmos quatro testes foram repetidos sem a observação discrepante identificada no gráfico dos resíduos condicionais padronizados, apresentado na Figura 4.8, sob o modelo normal com um efeito aleatório no intercepto. Podemos notar que, assumindo D diagonal o modelo normal apresenta mudança de decisão quanto à inclusão do efeito aleatório na inclinação na presença do efeito aleatório no intercepto (a um nível de significância de 5\%), enquanto que para o modelo exponencial potência esse teste foi robusto e preservou a decisão de incluir o efeito aleatório na inclinação. Considerando a matriz $\mathbf{D}$ não-diagonal, ambos os modelos indicaram que o efeito aleatório na inclinação deve ser incluído. Portanto, o modelo exponencial potência apresentou a mesma conclusão em todas as situações consideradas enquanto que o modelo normal foi afetado pela escolha da matriz $\mathbf{D}$ diagonal ou não.

Os parâmetros estimados sob o modelo normal com efeitos aleatórios no intercepto e inclinação estão apresentados na Tabela 4.6. Note que, para todos os parâmetros, os erros-padrão são menores sob o modelo exponencial potência, e portanto, há indícios de que as estimativas são, novamente, mais precisas do que sob o modelo normal.

A Figura 4.10 descreve os perfis individuais ajustados no modelo com dois efeitos aleatórios no intercepto e inclinação sob a suposição de distribuição exponencial potência ajustado para os dados da altura do ramo da mandíbula.

Os pesos $\hat{v}_{i}$ 's estão apresentados na Tabela 4.7. Note que o menino 12 , que apresentou uma observação discrepante recebeu peso menor do que os outros. A Figura 4.11 mostra que os pontos com menores pesos $\hat{v}_{i}$ 's são os que apresentam maiores resíduos.

Observando os gráficos da Figura 4.9, verificamos que para o caso normal nenhuma observação foi identificada como ponto de alavanca. Entretanto, no modelo exponencial potência, identificamos alguns pontos de alavanca que exercem 


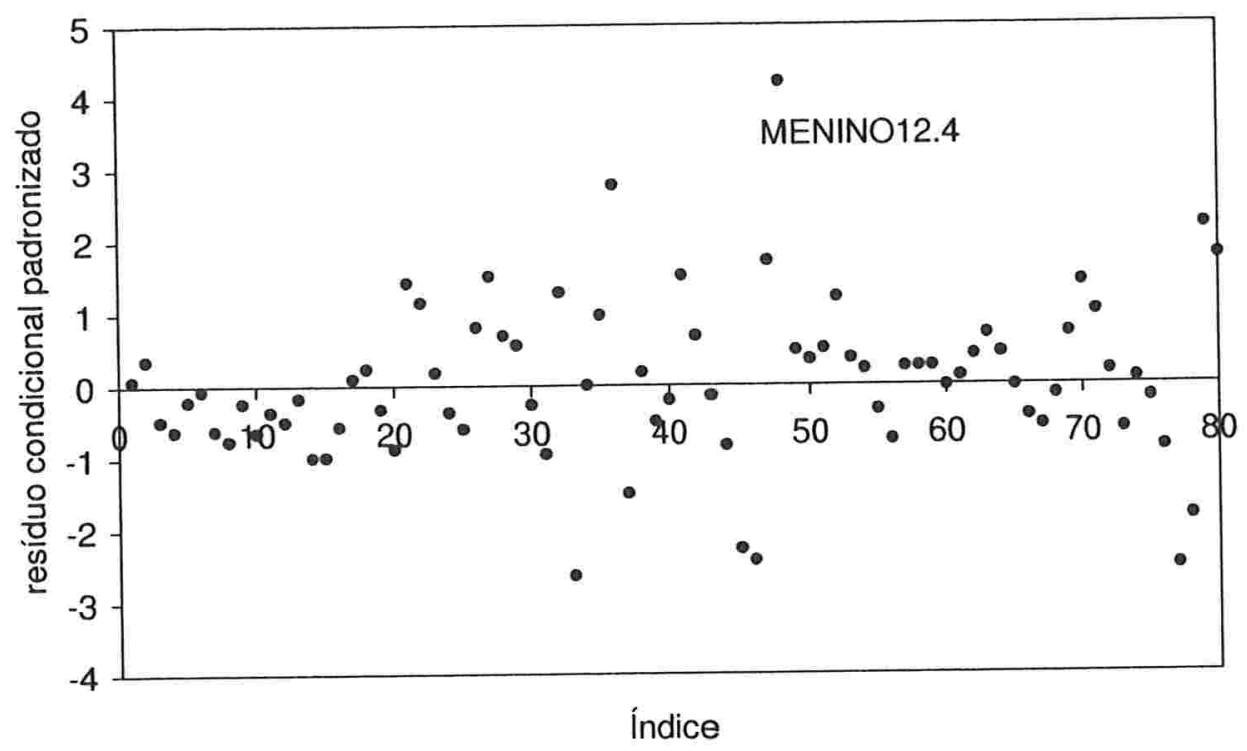

Figura 4.8: Gráfico dos resíduos condicionais padronizados para o modelo normal com um efeito aleatório no intercepto ajustado para os dados da altura do ramo da mandíbula.

uma forte influência nos seus próprios valores preditos. A Figura 4.12 confirma que esses pontos são os que apresentam maiores pesos. Portanto, neste exemplo a suposição de uma distribuição com caudas mais pesadas do que as da normal, apesar de controlar a contribuição do ponto discrepante no processo de estimação dos parâmetros, acarretou uma influência desproporcional das observações nas predições.

\subsection{Dados da terapia antiretroviral}

Como já vimos na introdução, os perfis individuais dos pacientes apresentados na Figura 1.3 mostram que a contagem de células CD4+ se altera muito pouco antes da introdução da terapia HAART, entretanto, após a introdução da HAART essa contagem parece crescer rapidamente, indicando uma importante melhora do sistema imunológico dos pacientes. Uma reta segmentada com ponto de inflexão no momento da introdução da nova terapia foi proposta em Borges et al. (2001). 
Tabela 4.5: Resultados da estatística $\mathrm{T}_{S}$ (p-valor) para as hipóteses consideradas sob o modelo normal e exponencial potência ajustados para os dados da altura do ramo da mandíbula.

\begin{tabular}{lcccc}
\hline \hline Hipóteses & Normal & \multicolumn{2}{c}{ EP $(0,6)$} \\
& $\begin{array}{c}\text { Dados } \\
\text { Completos }\end{array}$ & $\begin{array}{c}\text { Excluindo } \\
12.4\end{array}$ & $\begin{array}{c}\text { Dados } \\
\text { Completos }\end{array}$ & $\begin{array}{c}\text { Excluindo } \\
12.4\end{array}$ \\
\hline $\mathrm{H}_{0}: \tau_{1}=0$ & $96,2\left(<0,01^{1}\right)$ & $100,7\left(<0,01^{1}\right)$ & $102,0\left(<0,01^{1}\right)$ & $105,4\left(<0,01^{1}\right)$ \\
$\mathrm{H}_{1}: \tau_{1}>0$ & & & & \\
\hline $\mathrm{H}_{0}: \tau_{2}=0$ & $96,2\left(<0,01^{1}\right)$ & $100,4\left(<0,01^{1}\right)$ & $102,6\left(<0,01^{1}\right)$ & $105,5\left(<0,01^{1}\right)$ \\
$\mathrm{H}_{1}: \tau_{2}>0$ & & & & \\
\hline $\mathrm{H}_{0}: \tau_{2}=0, \mathbf{D}=\tau_{1}$ & $4,0\left(0,02^{1}\right)$ & $1,8\left(0,09^{1}\right)$ & \\
$\mathrm{H}_{1}: \tau_{2}>0$ & & & \\
$\mathbf{D}=\left[\begin{array}{cc}\tau_{1} & 0 \\
0 & \tau_{2}\end{array}\right]$ & & & & \\
\hline $\mathrm{H}_{0}: \tau_{2}=0, \mathbf{D}=\tau_{1}$ & $31,3\left(<0,01^{2}\right)$ & $17,4\left(<0,01^{1}\right)$ & \\
$\mathrm{H}_{1}: \tau_{2}>0$ & & & & \\
$\mathbf{D}=\left[\begin{array}{cc}\tau_{1} & \tau_{12} \\
\tau_{12} & \tau_{2}\end{array}\right]$ & & & & \\
\hline \hline
\end{tabular}

(1) Calculado com base na distribuição $\frac{1}{2} \chi_{0}^{2}+\frac{1}{2} \chi_{1}^{2}$.

(2) Calculado com base na distribuição $\frac{1}{2} \chi_{1}^{2}+\frac{1}{2} \chi_{2}^{2}$.

Tabela 4.6: Estimativas dos parâmetros dos modelos normal e exponencial potência com efeitos aleatórios no intercepto e inclinação e matriz $\mathbf{D}$ não-diagonal ajustados para os dados da altura do ramo da mandíbula.

\begin{tabular}{lcc}
\hline \hline & Normal & $\mathrm{EP}(0,6)$ \\
Parâmetro & Estimativa (erro-padrão) & Estimativa (erro-padrão) \\
\hline$\alpha$ - Intercepto & $33,75(2,24)$ & $35,97(0,65)$ \\
$\beta$ - Inclinação & $1,87(0,26)$ & $1,59(0,07)$ \\
\hline$\sigma^{2}$ & $0,19(0,04)$ & $0,03(0,01)$ \\
$\tau_{1}$ & $88,84(31,98)$ & $13,36(5,35)$ \\
$\tau_{12}$ & $-9,82(3,60)$ & $-1,43(0,59)$ \\
$\tau_{2}$ & $1.16(0,42)$ & $0,17(0,07)$ \\
\hline \hline
\end{tabular}



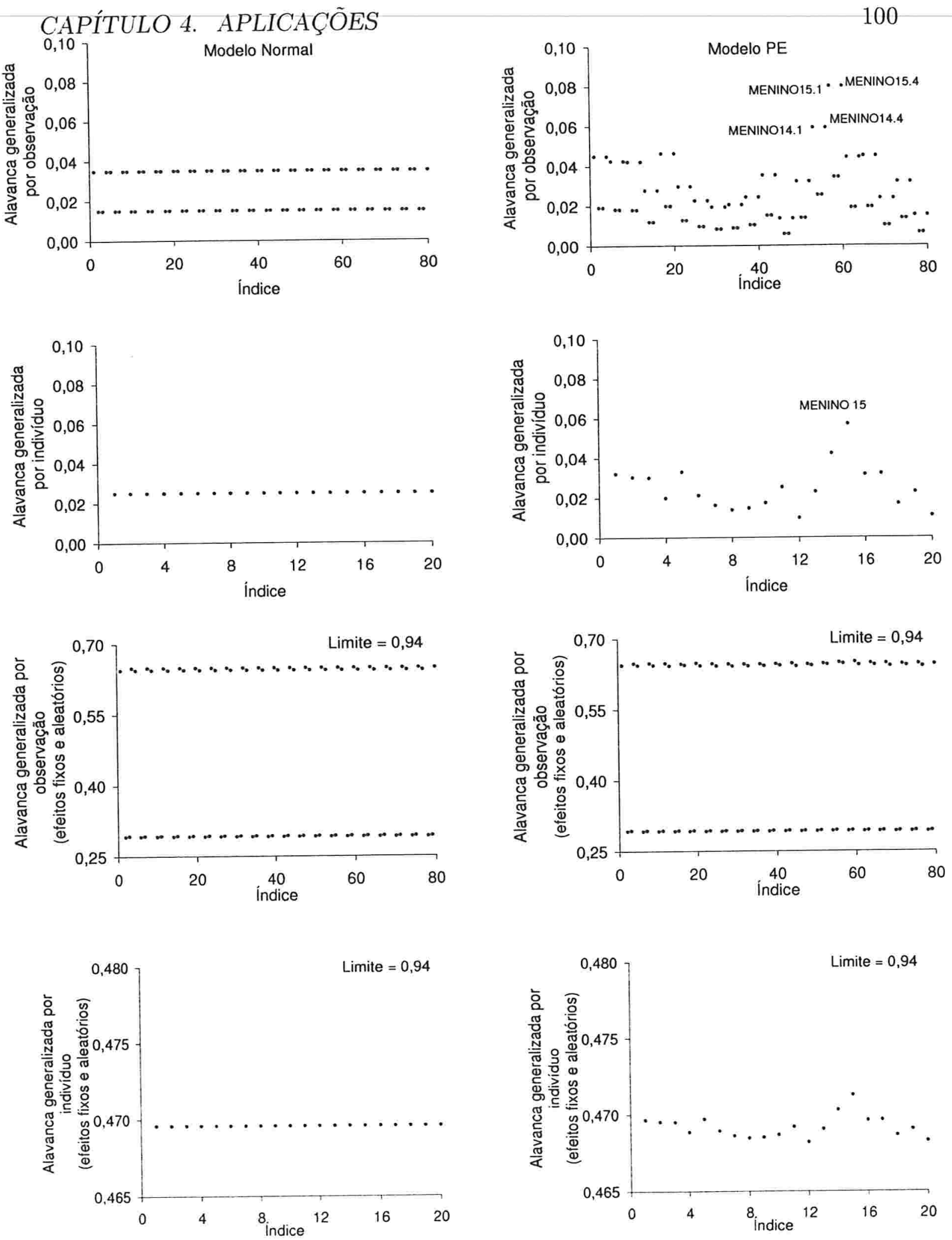

Figura 4.9: Gráficos da alavanca generalizada para o modelo final assumindo distribuição exponencial potência ajustado para os dados da altura do ramo da mandíbula. 
Tabela 4.7: Pesos estimados $v\left(\hat{u}_{i}\right)$ 's para o modelo com efeitos aleatórios no intercepto e inclinação assumindo distribuição exponencial potência para os dados da altura do ramo da mandíbula.

\begin{tabular}{cc|cc|cc|cc}
\hline \hline Menino & $v\left(\hat{u}_{i}\right)$ & Menino & $v\left(\hat{u}_{i}\right)$ & Menino & $v\left(\hat{u}_{i}\right)$ & Menino & $v\left(\hat{u}_{i}\right)$ \\
\hline 1 & 0,34 & 6 & 0,22 & 11 & 0,27 & 16 & 0,33 \\
2 & 0,32 & 7 & 0,17 & $\mathbf{1 2}$ & $\mathbf{0 , 1 0}$ & 17 & 0,34 \\
3 & 0,32 & 8 & 0,15 & 13 & 0,24 & 18 & 0,18 \\
4 & 0,21 & 9 & 0,16 & 14 & 0,45 & 19 & 0,24 \\
5 & 0,35 & 10 & 0,18 & 15 & 0,60 & 20 & 0,12 \\
\hline \hline
\end{tabular}

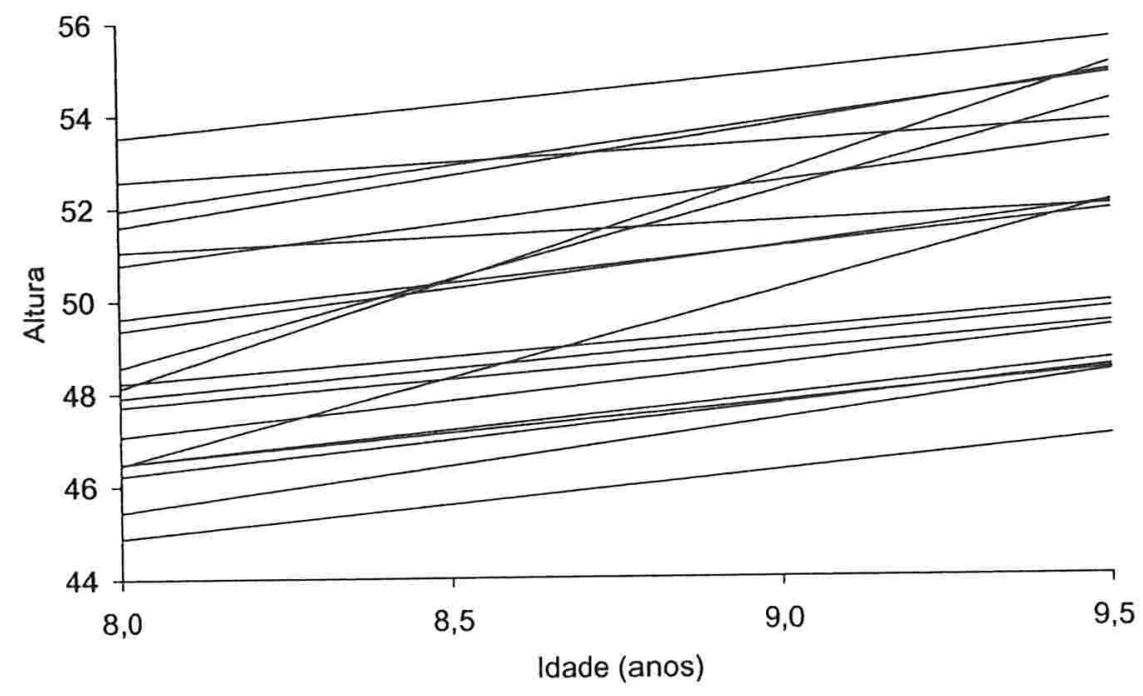

Figura 4.10: Perfis individuais ajustados para o modelo com dois efeitos aleatórios no intercepto e inclinação sob a suposição de distribuição exponencial potência ajustado para os dados da altura do ramo da mandíbula. 


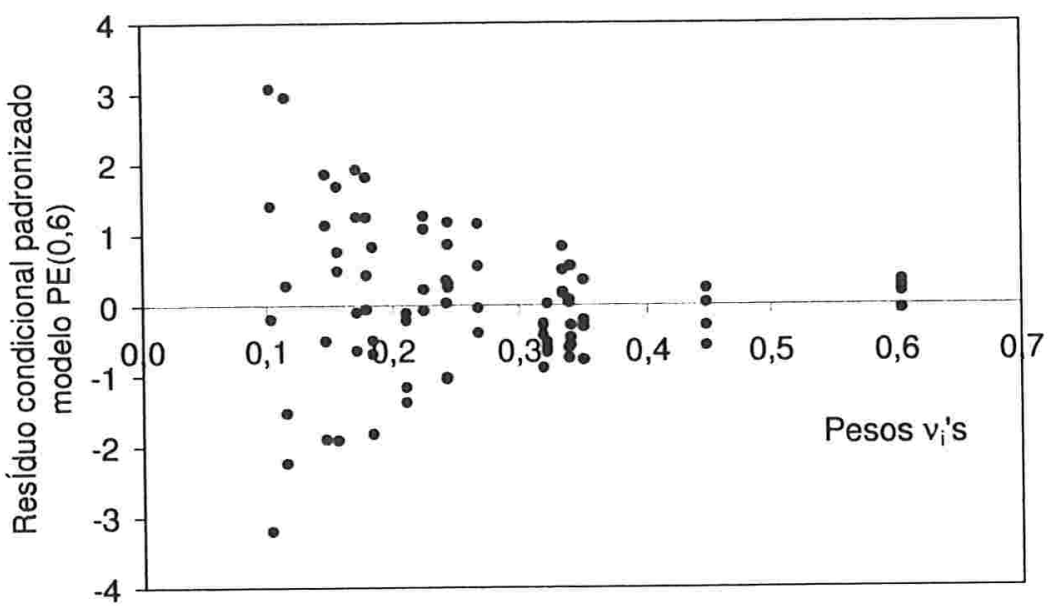

Figura 4.11: Gráfico dos pesos pelos resíduos condicionais padronizados para o modelo com dois efeitos aleatórios no intercepto e inclinação sob a suposição de distribuição exponencial potência ajustado para os dados da altura do ramo da mandíbula.

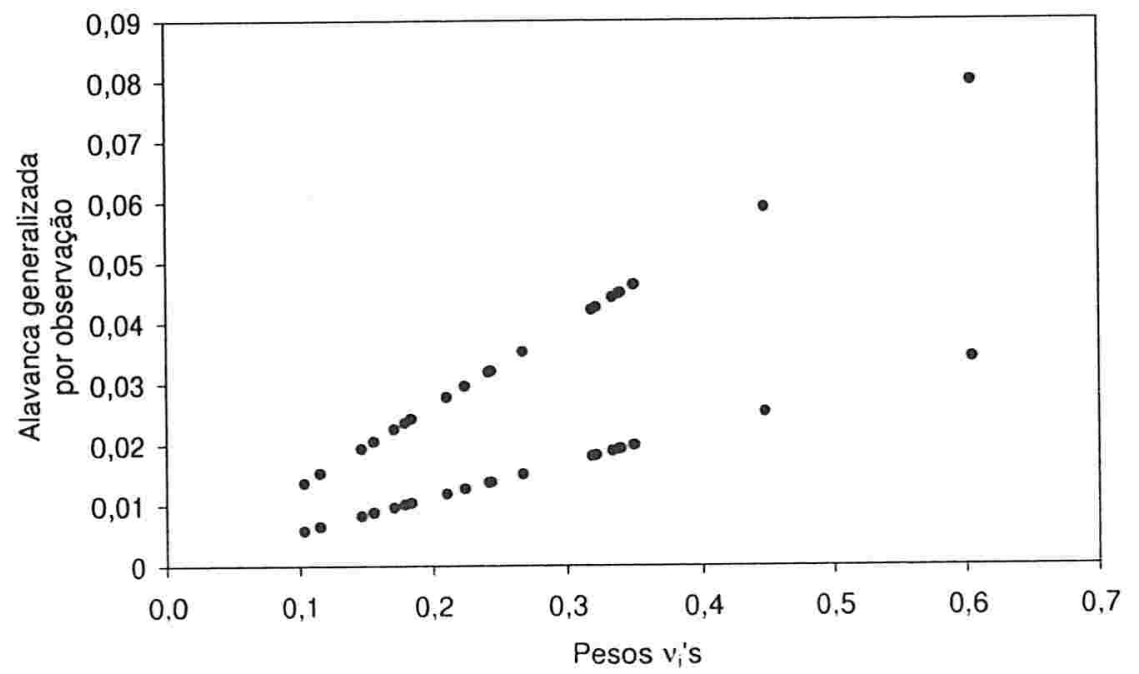

Figura 4.12: Gráfico dos pesos pela alavanca generalizada para o modelo com dois efeitos aleatórios no intercepto e inclinação sob a suposição de distribuição exponencial potência ajustado para os dados da altura do ramo da mandíbula. 
Como em geral as contagens não são pequenas para cada paciente (vide tabela 1.3) vamos propor para explicar $\mathbf{y}_{i}$ o modelo marginal dado abaixo

$$
\mathrm{y}_{i}=\mathbf{X}_{i} \boldsymbol{\beta}+\boldsymbol{\xi}_{i}, i=1, \ldots, 39
$$

em que $\boldsymbol{\xi}_{i} \sim \mathrm{El}_{3}\left(\mathbf{0} ; \mathbf{Z}_{i} \mathbf{D} \mathbf{Z}_{i}^{T}+\sigma^{2} \mathbf{I}_{m_{i}}\right)$

Contudo, modelos lineares generalizados mistos (ver, por exemplo, McCulloch e Searle, 2001), assumindo que $\mathbf{y}_{i} \mid \mathbf{x}_{i}$ segue uma distribuição de Poisson poderiam ser mais apropriados para analisar esse conjunto de dados.

Para pacientes com 3 observações, a primeira antes da terapia, a segunda no momento da introdução da HAART, considerado como tempo 0, e a última após o momento 0 , temos

$$
\begin{gathered}
\mathbf{y}_{i}=\left[\begin{array}{l}
y_{b} \\
y_{0} \\
y_{a}
\end{array}\right], \\
\mathbf{X}_{i}=\left[\begin{array}{ccc}
t_{b} & 1 & 0 \\
0 & 1 & 0 \\
0 & 1 & t_{a}
\end{array}\right]
\end{gathered}
$$

e $\boldsymbol{\beta}=\left(\beta_{b}, \alpha_{0}, \beta_{a}\right)^{T}$. Os parâmetros fixos foram indexados por $b$ para medidas antes da introdução da HAART ("before"), 0 para o tempo zero, e a para medidas após a introdução da HAART ("after"). Para modelar a dependência entre as medidas feitas no mesmo paciente, três possíveis estruturas para a matriz de variâncias-covariâncias foram consideradas:

(i) Modelo com um efeito aleatório no intercepto: $\mathbf{Z}_{i}=\mathbf{1}_{3}$ e $\mathbf{D}=\tau_{1}$;

(ii) Modelo com um efeito aleatório na inclinação (após a introdução do HA- 
$\mathrm{ART}): \mathbf{Z}_{i}=\left(0,0, t_{a}\right)^{T}$ e $\mathbf{D}=\tau_{2}$

(iii) Modelo com 2 efeitos aleatórios, no intercepto e na inclinação: $\mathbf{Z}_{i}=$ $\left[\begin{array}{ccc}1 & 1 & 1 \\ 0 & 0 & t_{a}\end{array}\right]^{T}$ e $\mathbf{D}=\operatorname{diag}\left[\tau_{1}, \tau_{2}\right]$

Novamente, ajustando retas individuais para cada paciente calculamos o coeficiente de correlação entre o intercepto e a inclinação após a introdução da terapia, que foi de $-0,17$, indicando que não parece haver correlação, assim, só consideramos o caso da matriz $\mathbf{D}$ diagonal.

Inicialmente, ajustamos o modelo com efeito aleatório na inclinação após a introdução da terapia, sob a suposição de distribuição normal para os erros, como proposto em Borges et al. (2001). O gráfico dos resíduos condicionais padronizados para esse modelo foi construído e está apresentado na Figura 4.14. Embora os resíduos apresentem uma distribuição ligeiramente assimétrica é possível identificar 3 observações que apresentam valores aberrantes, e portanto, uma distribuição com caudas mais pesadas para os erros pode ser considerada para acomodar uma maior variação nos dados. Para ilustrar, utilizamos novamente a distribuição tStudent com 3 graus de liberdade, escolhida pelo critério da informação de Akaike apresentado na Figura 4.13.

Para as duas distribuições consideradas o modelo de independência foi testado contra os modelos (i), (ii) e (iii) utilizando a estatística $T_{S}$. Uma outra situação foi considerada de tal forma a testar a necessidade de inclusão do efeito aleatório no intercepto quando o efeito aleatório da inclinação já está no modelo. Os valores da estatística $T_{S}$ para os dois modelos com e sem as observações destacadas estão apresentados na Tabela 4.8. Considerando um nível de significância de $10 \%$. note que sob a suposição de normalidade a decisão para o último teste muda na presença, ou não, das observações destacadas. Entretanto, para o modelo com caudas mais pesadas do que as da normal, essa conclusão se mantém, indicando que o efeito aleatório no intercepto também deve ser incluído no modelo, jun- 


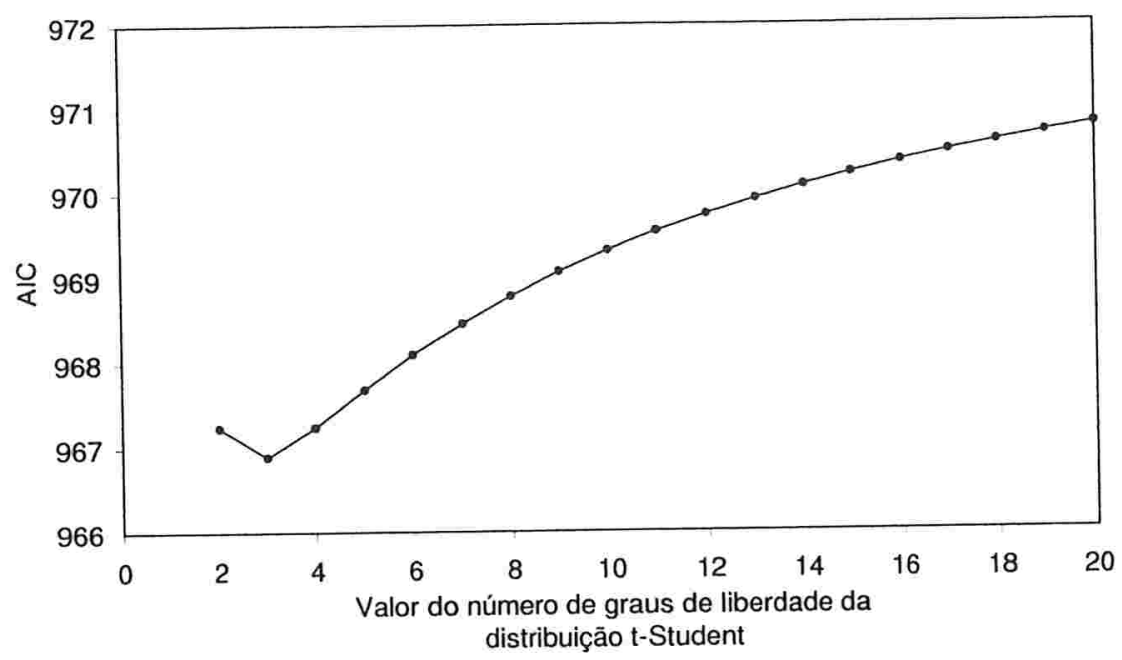

Figura 4.13: Critério da informação de Akaike para vários valores do número de graus de liberdade da distribuição t-Student para os dados da terapia antiretroviral.

tamente com o efeito aleatório na inclinação. Portanto, novamente temos um exemplo em que a suposição de uma distribuição com caudas mais pesadas do que as da normal para acomodar observações aberrantes pode acarretar mudança de decisão na escolha da matriz de variâncias-covariâncias.

A influência da retirada das observações destacadas nas estimativas dos parâmetros do modelo final com dois efeitos aleatórios, assim como na estatística $\mathrm{T}_{S}$ foi avaliada e está apresentada na Tabela 4.9. As variações são, geralmente, menores sob a suposição de distribuição t-Student.

As estimativas do modelo final sob a suposição de normalidade e de distribuição t-Student com 3 graus de liberdade para o modelo com dois efeitos aleatórios estão apresentadas na Tabela 4.10 e a Figura 4.15 mostra os perfis individuais ajustados para o modelo t-Student final. Note que os erros-padrão sob suposição de distribuição t-Student são, em geral, menores, confirmando os resultados dos exemplos anteriores de que, na presença de pontos aberrantes, a suposição de uma distribuição com caudas mais pesadas do que as da normal produz estimativas 


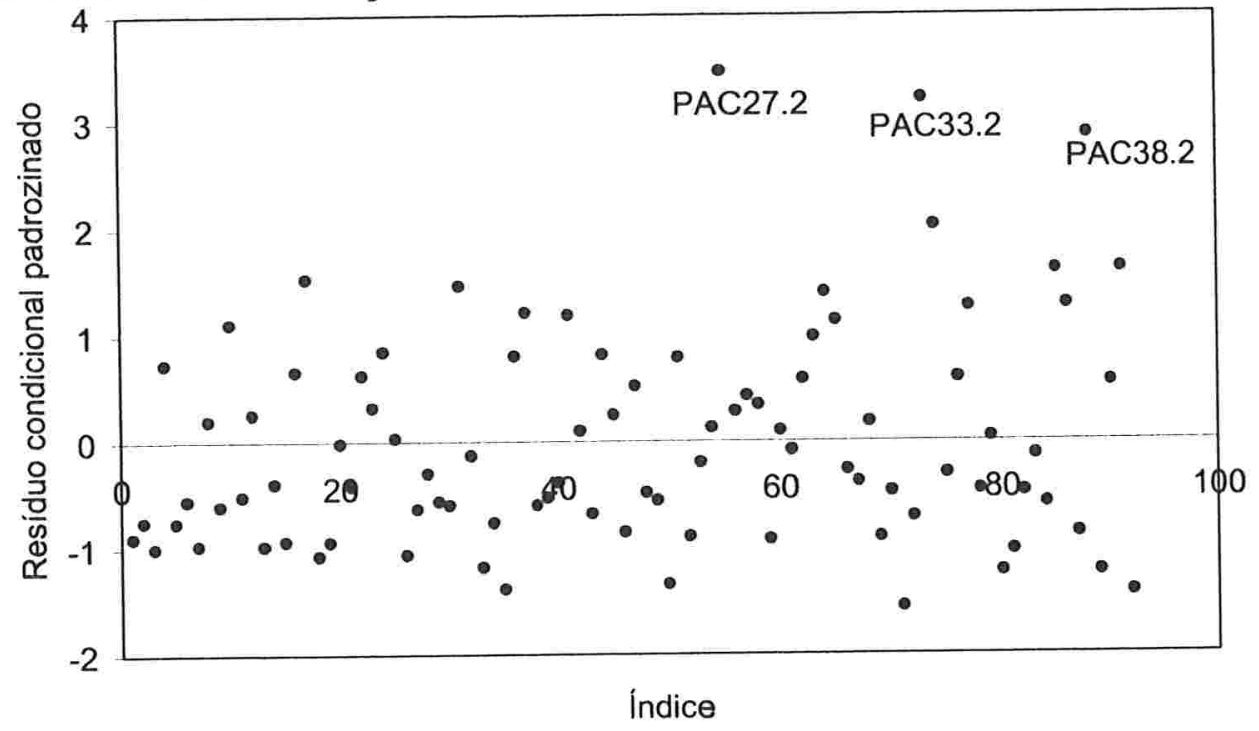

Figura 4.14: Gráfico dos resíduos condicionais padronizados para o modelo normal com efeito aleatório na inclinação após a introdução da HAART ajustado para os dados da terapia antiretroviral.

mais precisas.

Os pesos $\hat{v}_{i}$ 's estimados sob o modelo t-Student com 3 graus de liberdade estão apresentados na Tabela 4.11. Note que os pacientes com observações aberrantes receberam pesos menores do que os outros.

A Figura 4.16 mostra os gráficos dos pontos de alavanca e podemos concluir que, tanto no modelo normal quanto no modelo t-Student, várias observações de vários pacientes são consideradas pontos de alavanca, principalmente nos efeitos fixos e aleatórios. Isso se deve ao fato de que a variável explicativa tempo assume vários valores atípicos, refletindo na matriz de projeção. Entretanto, no modelo t-Student isso pode ser atribuído também ao fato de que esses pontos receberam maiores pesos, como mostra a Figura 4.18, diferente do modelo normal em que todas as observações recebem pesos iguais a 1 .

Esses dados foram ajustados, originalmente, utilizando um modelo misto normal dada a sua natureza desbalanceada, e aqui procuramos uma forma de melhorar o ajuste acomodando as várias observações aberrantes. Entretanto, a variável 
Tabela 4.8: Resultados da estatística $\mathrm{T}_{S}$ (p-valor) para as hipóteses consideradas sob o modelo normal e t-Student com 3 graus de liberdade ajustados para os dados da terapia antiretroviral.

\begin{tabular}{lrrrr}
\hline \hline Hipóteses & Normal & t-Student & $(3$ g.l. $)$ \\
\hline & $\begin{array}{r}\text { Dados } \\
\text { completos }\end{array}$ & $\begin{array}{r}\text { Sem obs. } \\
\text { destacadas }\end{array}$ & $\begin{array}{r}\text { Dados } \\
\text { completos }\end{array}$ & $\begin{array}{r}\text { Sem obs. } \\
\text { destacadas }\end{array}$ \\
\hline $\mathrm{H}_{0}: \tau_{1}=0$ & $0,39\left(0,27^{1}\right)$ & $0,18\left(0,34^{1}\right)$ & $1,02\left(0,16^{1}\right)$ & $1,20\left(0,14^{1}\right)$ \\
$\mathrm{H}_{1}: \tau_{1}>0$ & & & & \\
\hline $\mathrm{H}_{0}: \tau_{2}=0$ & $65,54\left(<0,01^{1}\right)$ & $79,62\left(<0,01^{1}\right)$ & $52,47\left(<0,01^{1}\right)$ & $60,64\left(<0,01^{1}\right)$ \\
$\mathrm{H}_{1}: \tau_{2}>0$ & & & & \\
\hline $\mathrm{H}_{0}: \tau_{1}=\tau_{2}=0$ & $65,95\left(<0,01^{2}\right)$ & $79,82\left(<0,01^{2}\right)$ & $53,53\left(<0,01^{2}\right)$ & $61,88\left(<0,01^{2}\right)$ \\
$\mathrm{H}_{1}: \tau_{1}>0$ & & & & \\
and $/$ or $\tau_{2}>0$ & & & $1,76\left(0,09^{1}\right)$ & $3,33\left(0,03^{1}\right)$ \\
\hline $\mathrm{H}_{0}: \tau_{1}=0$ & $0,30\left(0,29^{1}\right)$ & $4,33\left(0,02^{1}\right)$ & & \\
$\mathrm{H}_{1}: \tau_{1}>0$ & & & & \\
com $\tau_{2}$ no modelo & & & & \\
\hline \hline
\end{tabular}

(1) Calculado com base na distribuição $\frac{1}{2} \chi_{0}^{2}+\frac{1}{2} \chi_{1}^{2}$.

(2) Calculado com base na distribuição $\frac{1}{4} \chi_{0}^{2}+\frac{1}{2} \chi_{1}^{2}+\frac{1}{4} \chi_{2}^{2}$.

Tabela 4.9: Variações nas estimativas dos parâmetros e na estatística $\mathrm{T}_{S}$ do modelo com efeitos aleatórios na inclinação e intercepto sob a suposição de distribuição normal e t-Student com 3 graus de liberdade ajustado para os dados da terapia antiretroviral, após excluir as observações destacadas na Figura 4.14.

\begin{tabular}{lrr}
\hline \hline & Normal & t-Student(3g.l.) \\
\hline & Excluindo & Excluindo \\
& $(27.2,33.2,38.2)$ & $(27.2,33.2,38.2)$ \\
\hline$\beta_{b}$ - inclinação antes da HAART & $-174,8 \%$ & $28,5 \%$ \\
$\alpha_{0}$ - Intercepto & $-20,8 \%$ & $-12,9 \%$ \\
$\beta_{a}$ - inclinação após a HAART & $6,3 \%$ & $3,5 \%$ \\
\hline$\sigma^{2}$ & $-63,4 \%$ & $-41,7 \%$ \\
$\tau_{1}$ & $164,2 \%$ & $12,9 \%$ \\
$\tau_{2}$ & $6,8 \%$ & $8.7 \%$ \\
\hline \hline
\end{tabular}


Tabela 4.10: Estimativas dos parâmetros do modelo com efeitos aleatórios na inclinação e intercepto sob a suposição de distribuição normal e t-Student com 3 graus de liberdade para os dados da terapia antiretroviral.

\begin{tabular}{lrr}
\hline \hline & $\begin{array}{r}\text { Normal } \\
\text { t-Student(3g.l.) }\end{array}$ \\
\hline Parâmetro & $\begin{array}{r}\text { Estimativa } \\
\text { (erro-padrão) }\end{array}$ & $\begin{array}{r}\text { Estimativa } \\
\text { (erro-padrão) }\end{array}$ \\
\hline$\beta_{b}$ - inclinação antes da HAART & $0,02(0,02)$ & $-0,02(0,02)$ \\
$\alpha_{0}$ - intercepto & $26,94(3,74)$ & $17,88(2,94)$ \\
$\beta_{a}$ - inclinação após da HAART & $0,26(0,04)$ & $0,26(0,04)$ \\
\hline$\sigma^{2}$ & $536,09(136,19)$ & $197,15(68,68)$ \\
$\tau_{1}$ & $39,71(103,91)$ & $54,32(56,54)$ \\
$\tau_{2}$ & $0,04(0,01)$ & $0,03(0,01)$ \\
\hline
\end{tabular}

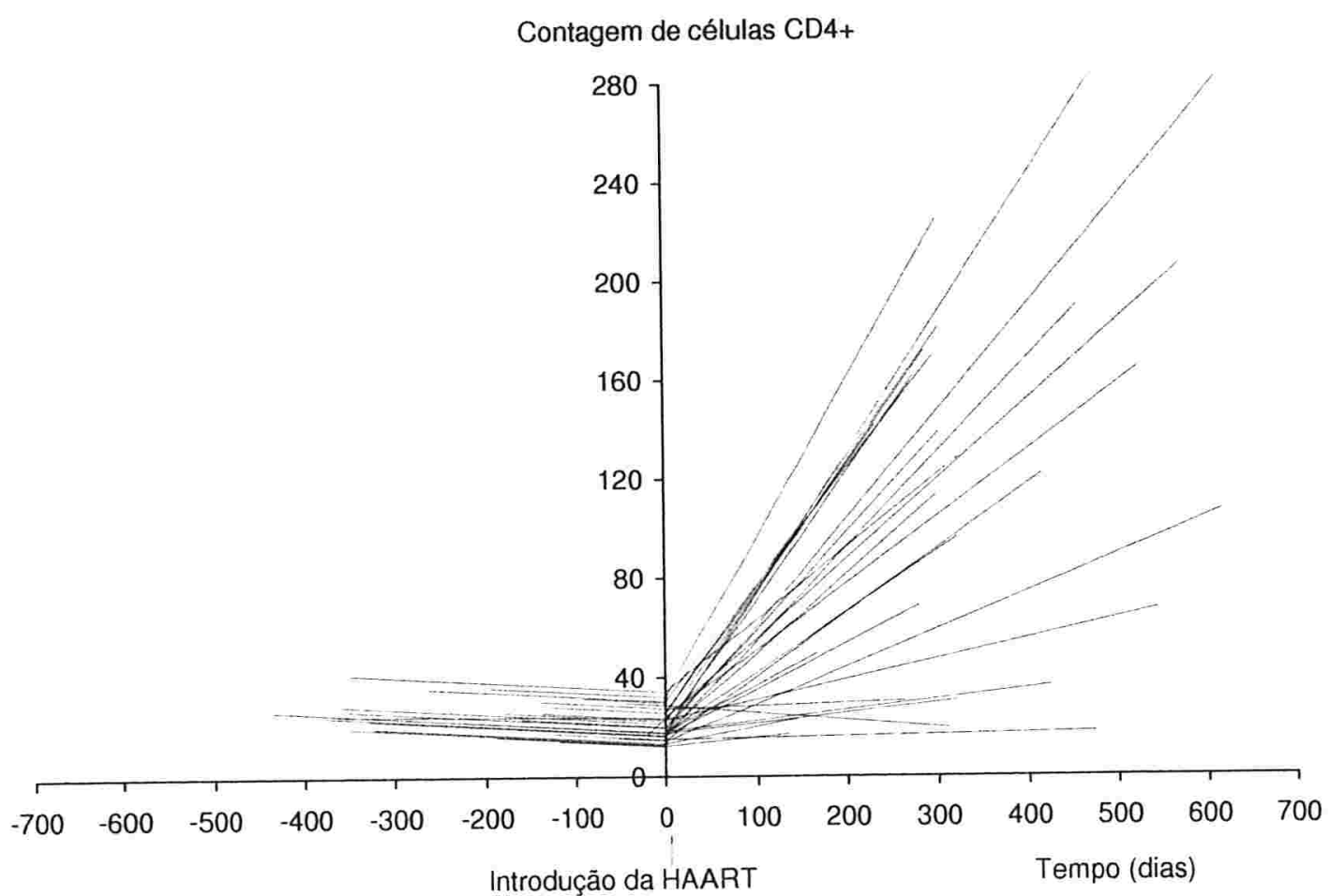

Figura 4.15: Perfis individuais ajustados para o modelo com efeitos aleatórios na inclinação e intercepto sob a suposição de distribuição t-Student com 3 graus de liberdade ajustado para os dados da terapia antiretroviral. 

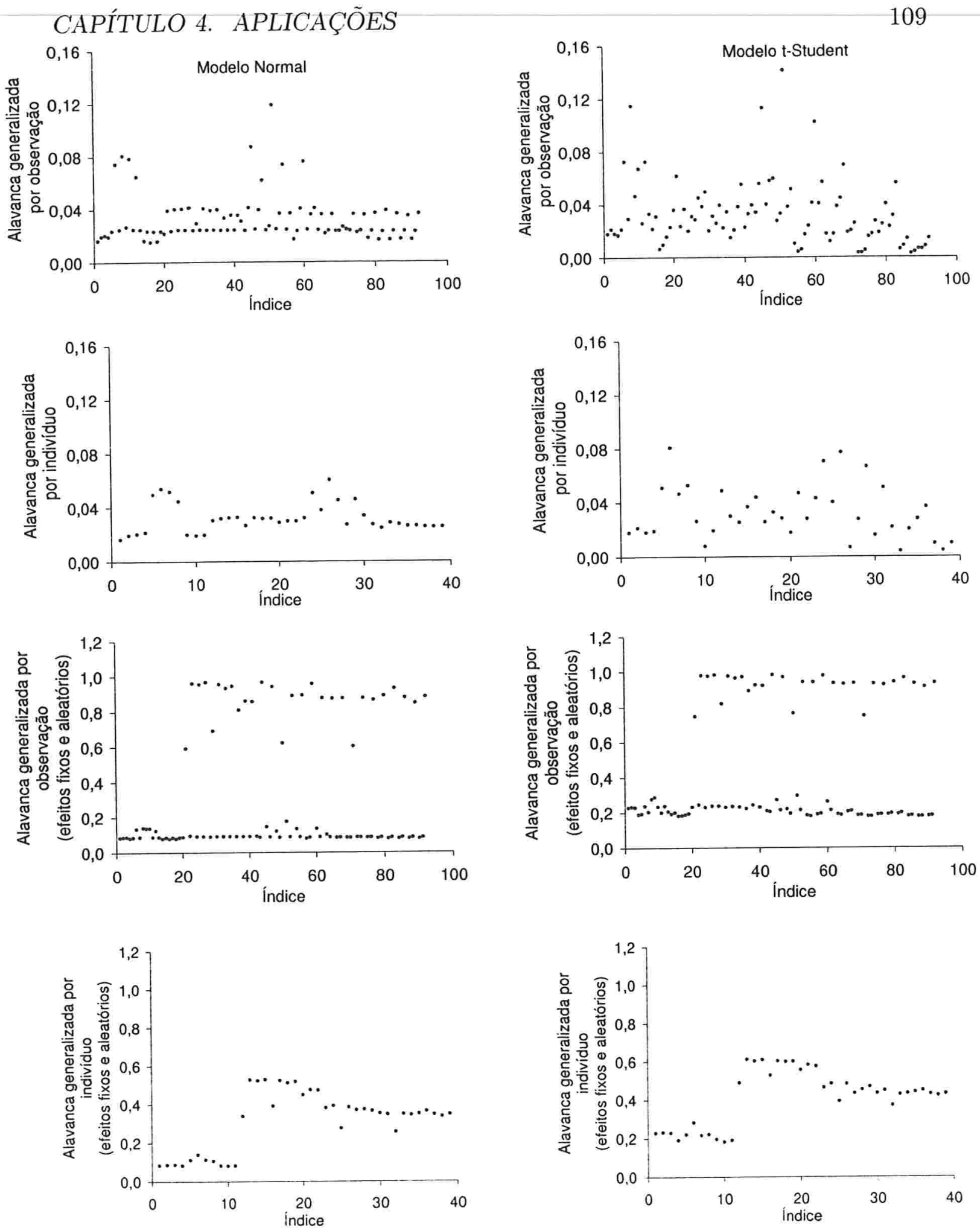

Figura 4.16: Gráficos da alavanca generalizada para o modelo com efeitos aleatórios na inclinação e intercepto sob a suposição de distribuição t-Student com 3 graus de liberdade ajustado para os dados da terapia antiretroviral. 
Tabela 4.11: Pesos estimados $v\left(\hat{u}_{i}\right)$ 's para o modelo com efeitos aleatórios na inclinação e intercepto sob a suposição de distribuição t-Student com 3 graus de liberdade para os dados da terapia antiretroviral.

\begin{tabular}{cc|cc|cc|cc}
\hline \hline PAC & $v\left(\hat{u}_{i}\right)$ & PAC & $v\left(\hat{u}_{i}\right)$ & PAC & $v\left(\hat{u}_{i}\right)$ & PAC & $v\left(\hat{u}_{i}\right)$ \\
\hline 1 & 0,99 & 11 & 1,01 & 21 & 1,51 & 31 & 1,88 \\
2 & 1,06 & 12 & 1,65 & 22 & 0,90 & 32 & 0,86 \\
3 & 0,86 & 13 & 0,94 & 23 & 1,39 & $\mathbf{3 3}$ & $\mathbf{0 , 1 4}$ \\
4 & 0,91 & 14 & 0,78 & 24 & 1,49 & 34 & 0,74 \\
5 & 1,12 & 15 & 1,13 & 25 & 1,09 & 35 & 1,07 \\
6 & 1,62 & 16 & 1,55 & 26 & 1,38 & 36 & 1,44 \\
7 & 0,98 & 17 & 0,79 & $\mathbf{2 7}$ & $\mathbf{0 , 1 6}$ & 37 & 0,38 \\
8 & 1,27 & 18 & 1,02 & 28 & 1,04 & $\mathbf{3 8}$ & $\mathbf{0 , 1 8}$ \\
9 & 1,36 & 19 & 0,88 & 29 & 1,53 & 39 & 0,39 \\
10 & 0,43 & 20 & 0,59 & 30 & 0,48 & & \\
\hline \hline
\end{tabular}

contagem de células CD4+ apresenta uma forte assimetria, o que pode explicar a presença de tantos pontos alavancas. Métodos de modelos mistos assumindo uma distribuição mais adequada para dados de contagem ou uma distribuição assimétrica que permitam a presença de dados desbalanceados seriam alternativas provavelmente mais adequadas para esse caso. 


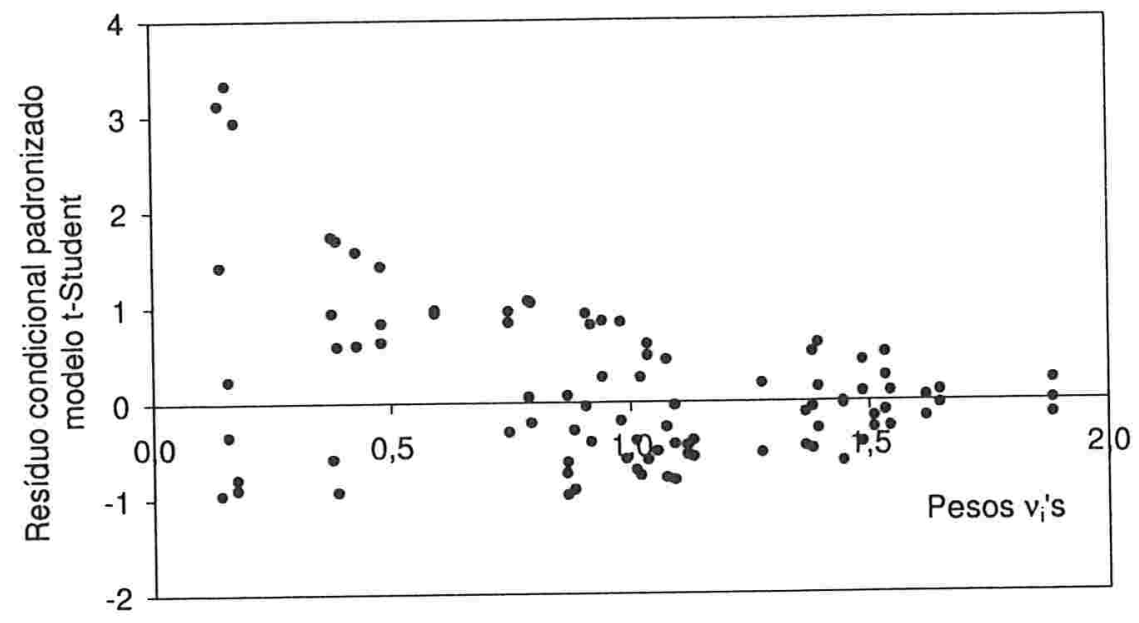

Figura 4.17: Gráfico dos pesos pelos resíduos condicionais padronizados para o modelo t-Student com dois efeitos aleatórios no intercepto e inclinação após a introdução da HAART ajustado para os dados da terapia antiretroviral.

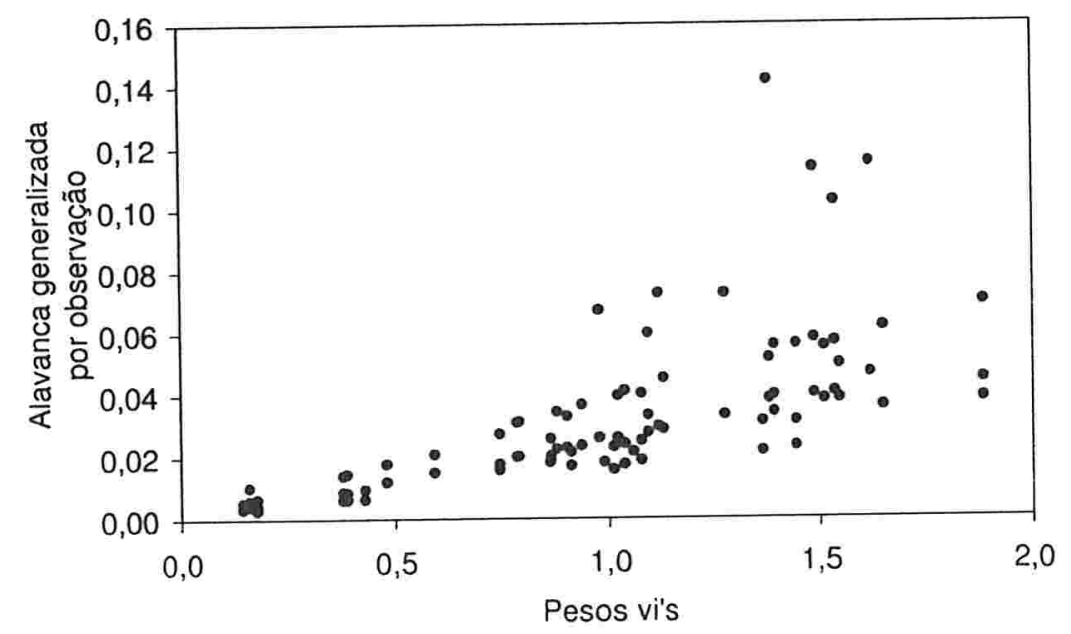

Figura 4.18: Gráfico dos pesos pela alavanca generalizada para o modelo tStudent com dois efeitos aleatórios no intercepto e inclinação após a introdução da HAART ajustado para os dados da terapia antiretroviral. 


\section{Capítulo 5}

\section{Estudos de simulação}

Nestes estudos de simulação o objetivo principal foi comparar o poder do teste tipo escore $\left(\mathrm{T}_{S}\right)$ proposto neste trabalho com o poder do teste da razão de verossimilhanças $(R V)$ para amostras pequenas e moderadas.

Por simplicidade, assumimos dados balanceados e com mesma estrutura dos dados de Potthoff-Roy, ou seja,

$$
\mathrm{y}_{i}=\mathrm{X}_{i} \boldsymbol{\beta}+\boldsymbol{\xi}_{i}, i=1, \ldots, n
$$

em que $n$ é o número de "clusters", fixado em 20, 40 ou 60. O vetor de parâmetros $\boldsymbol{\beta}$ foi pré-fixado, bem como as matrizes de planejamento para cada "cluster", isto é,

$$
\mathbf{X}_{i}=\left[\begin{array}{cccc}
1 & 8 & 0 & 0 \\
1 & 10 & 0 & 0 \\
1 & 12 & 0 & 0 \\
1 & 14 & 0 & 0
\end{array}\right]
$$

para um dos grupos, $i=1, \ldots, \frac{n}{2}$, e,

$$
\mathbf{X}_{i}=\left[\begin{array}{cccc}
0 & 0 & 1 & 8 \\
0 & 0 & 1 & 10 \\
0 & 0 & 1 & 12 \\
0 & 0 & 1 & 14
\end{array}\right]
$$


para o outro grupo, $i=\left(\frac{n}{2}+1\right), \ldots, n$.

Adicionalmente, assumimos $\xi_{i} \sim \mathrm{El}_{4}\left(0 ; \Sigma_{i}\right)$. As distribuições elípticas utilizadas foram a t-Student com 5 e 10 graus de liberdade e a exponencial potência $\operatorname{com} K=0,5$.

As seguintes estruturas para a matriz $\boldsymbol{\Sigma}_{i}$ foram adotadas:

(i) $\boldsymbol{\Sigma}_{i}=\sigma^{2} \mathbf{I}_{4}$, correspondente ao modelo de independência;

(ii) $\boldsymbol{\Sigma}_{i}=\tau \mathbf{J}_{4}+\sigma^{2} \mathbf{I}_{4}$ correspondente ao modelo com efeito aleatório no intercepto.

Os valores de $\sigma^{2}$ e do componente de variância também foram pré-fixados.

\subsection{Teste para um efeito aleatório no intercepto}

O interesse é testar a hipótese $\mathrm{H}_{0}: \tau=0$ contra $\mathrm{H}_{1}: \tau>0$. Assim, sob a hipótese nula, temos o modelo de independência e sob a hipótese alternativa o modelo com um efeito aleatório no intercepto. Os valores fixados para os parâmetros com os quais as distribuições t-Student multivariadas foram geradas são $\boldsymbol{\beta}=(17,0 ; 0,5 ; 16,0 ; 0,7)^{T}$ e $\sigma^{2}=3,5$, valores semelhantes aos estimados no conjunto de dados original. Para $\tau$, consideraram-se os valores 0,0 (tamanho do teste), $0,2,0,4,0,6,0,8,1,2,3$ e 4 .

Para cada configuração $\left(\boldsymbol{\beta}, \sigma^{2}, \tau\right)$ foram geradas 5000 réplicas para $\mathbf{y}_{i}(i=$ $1, \ldots, n)$ tais que

$$
\mathbf{y}_{i} \sim \mathrm{El}_{4}\left(\mathbf{X}_{i} \boldsymbol{\beta} ; \sigma^{2} \mathbf{I}_{4}\right)
$$

sob a hipótese nula, e,

$$
\mathbf{y}_{i} \sim \mathrm{El}_{4}\left(\mathbf{X}_{i} \boldsymbol{\beta} ; \tau \mathbf{J}_{4}+\sigma^{2} \mathbf{I}_{4}\right)
$$


sob a hipótese alternativa.

Para cada amostra gerada, foram calculados os valores das estatísticas da razão de verossimilhanças e do tipo escore. O poder dos testes foi estimado como sendo a proporção de p-valores maiores do que $\alpha$, o nível de significância do teste, que foi fixado em $1 \%, 5 \%$ ou $10 \%$. O valor de p para o teste da razão de verossimilhanças foi estimado por $p=\frac{1}{2} P\left(\chi_{1}^{2} \geq r_{v}\right)$, em que $r_{v}$ é o valor observado da estatística, enquanto que o valor de p para o teste tipo escore foi estimado por:

$$
p= \begin{cases}\frac{1}{2} P\left(\chi_{1}^{2} \geq r_{t}\right) & \text { se } r_{t}>0 \\ 0,5 & \text { se } r_{t}=0\end{cases}
$$

em que $r_{t}$ é o valor observado da estatística $\mathrm{T}_{S}$.

A Tabela 5.1 e as Figuras 5.1, 5.2 e 5.3 apresentam os resultados da simulação proposta considerando uma distribuição t-Student multivariada com 5 graus de liberdade. Observa-se que, em geral, a estatística da razão de verossimilhanças subestima o tamanho do teste enquanto que a estatística Ts superestima o mesmo. Não obstante, nota-se para ambas as estatísticas que, à medida que aumenta o valor do componente de variância aumenta o poder, como já era esperado. Entretanto, a estatística do tipo escore $T_{S}$ apresenta valores maiores para o poder em todas as situações, mostrando-se discretamente melhor, ou no mínimo tão poderosa quanto a estatística da razão de verossimilhanças para amostras pequenas. Adicionalmente, à medida que aumenta o tamanho da amostra os valores do poder das duas estatísticas ficam mais próximos, indicando que a teoria assintótica já verificada para o caso normal por Silvapulle e Silvapulle (1995) também se confirma empiricamente para a distribuição elíptica aqui adotada.

A Tabela 5.2 e as Figuras 5.4, 5.5 e 5.6 apresentam os resultados da simulação proposta, considerando uma distribuição t-Student multivariada com 10 graus de liberdade. As conclusões foram as mesmas obtidas com 5 graus de liberdade. Uma 
análise comparativa dos resultados das simulações para os números de graus de liberdade 5 e 10 mostra que, para a distribuição mais próxima da normal, a tStudent com 10 graus de liberdade, o poder é maior ou igual ao obtido com a distribuição t-Student com 5 graus de liberdade, para quase todas as situações consideradas.

A Tabela 5.3 e as Figuras 5.7, 5.8 e 5.9 apresentam os resultados da simulação proposta, considerando uma distribuição exponencial potência com $K=0,5$. Neste caso, em geral, as duas estatísticas superestimam o tamanho do teste e os resultados indicam que a estatística do tipo escore e da razão de verossimilhanças apresentam valores próximos para o poder em todas as situações.

É importante ressaltar que essa simulação se refere a uma situação específica, e, portanto, as conclusões são restritas a esse caso específico. Entretanto, para obter conclusões mais seguras acerca da superioridade do poder da estatística do tipo escore outros estudos de simulação relacionados a outras distribuições e hipóteses mais complexas devem ser propostos. 
Tabela 5.1: Valores estimados do poder para o teste de um componente de variância para dados gerados a partir de uma distribuição t-Student multivariada com 5 graus de liberdade.

\begin{tabular}{|c|c|c|c|c|c|c|c|c|c|c|c|}
\hline & & & & & & & $\tau$ & & & & \\
\hline$n$ & $\alpha$ & Estatística & 0,0 & 0,2 & 0,4 & 0,6 & 0,8 & 1,0 & 2,0 & 3,0 & 4,0 \\
\hline \multirow[t]{6}{*}{20} & $10 \%$ & $\overline{T_{S}}$ & 1010,0 & 21,6 & 35,2 & 49,0 & 60,7 & 71,1 & 92,7 & 98,3 & $\overline{99,6}$ \\
\hline & & $R V$ & 7,7 & 16,8 & 29,5 & 42,4 & 54,3 & 65,1 & 90,7 & 97,7 & 99,4 \\
\hline & $5 \%$ & $T_{S}$ & 6,2 & 13,7 & 25,8 & 37,6 & 49,1 & 61,3 & 88,8 & 96,8 & 99,2 \\
\hline & & $R V$ & 3,9 & 9,3 & 19,3 & 29,9 & 40,3 & 52,6 & 84,9 & 95,3 & 98,7 \\
\hline & $1 \%$ & $T_{S}$ & 1,9 & 5,0 & 12,2 & 20,8 & 30,2 & 40,6 & 77,6 & 92,1 & 97,5 \\
\hline & & $R V$ & 0,9 & 2,3 & 5,9 & 12,8 & 19,5 & 28,8 & 67,1 & 87,2 & 95,4 \\
\hline \multirow[t]{6}{*}{40} & $10 \%$ & $\overline{T_{S}}$ & 10,9 & 29,2 & 51,6 & 70,7 & 83,2 & 91,3 & 99,7 & 100,0 & 100,0 \\
\hline & & $R V$ & 8,2 & 24,1 & 45,3 & 65,4 & 79,1 & 88,6 & 99,6 & 100,0 & 100,0 \\
\hline & $5 \%$ & $\overline{T_{S}}$ & 6,3 & 20,1 & 39,7 & 60,3 & 75,1 & 85,6 & 99,3 & 100,0 & 100,0 \\
\hline & & $R V$ & 4,0 & 14,5 & 32,4 & 52,6 & 68,6 & 79,9 & 98,8 & 100,0 & 100,0 \\
\hline & $1 \%$ & $T_{S}$ & 1,9 & 8,3 & 21,8 & 39,3 & 57,1 & 70,4 & 97,3 & 99,9 & 100,0 \\
\hline & & $R V$ & 0,7 & 4,1 & 14,2 & 28,2 & 44,9 & 59,1 & 95,4 & 99,6 & 100,0 \\
\hline \multirow[t]{6}{*}{60} & $10 \%$ & $\overline{\overline{T_{S}}}$ & 111,2 & 35,6 & 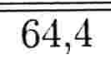 & 83,3 & $\overline{93,4}$ & 97,4 & 100,0 & 100,0 & 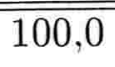 \\
\hline & & $R V$ & 8,3 & 30,4 & 58,6 & 79,5 & 91,1 & 96,5 & 100,0 & 100,0 & 100,0 \\
\hline & $5 \%$ & $T_{S}$ & 6,2 & 25,7 & 52,7 & 75,4 & 88,7 & 95,3 & 100,0 & 100,0 & 100,0 \\
\hline & & $R V$ & 4,3 & 19,7 & 45,2 & 68,9 & 84,6 & 93,3 & 99,9 & 100,0 & 100,0 \\
\hline & $1 \%$ & $T_{S}$ & 1,8 & 11,5 & 32,2 & 56,0 & 75,2 & 88,2 & 99,8 & 100,0 & 100,0 \\
\hline & & $R V$ & 0,8 & 6,6 & 22,4 & 44,3 & 64,7 & 81.0 & 99,7 & 100,0 & 100,0 \\
\hline
\end{tabular}


Figura 5.1: Gráficos do poder estimado das estatísticas $T_{S}$ e $R V$ para $n=20,40$ ou 60 e nível de significância de $10 \%$ para dados gerados a partir de uma distribuição t-Student multivariada com 5 graus de liberdade.
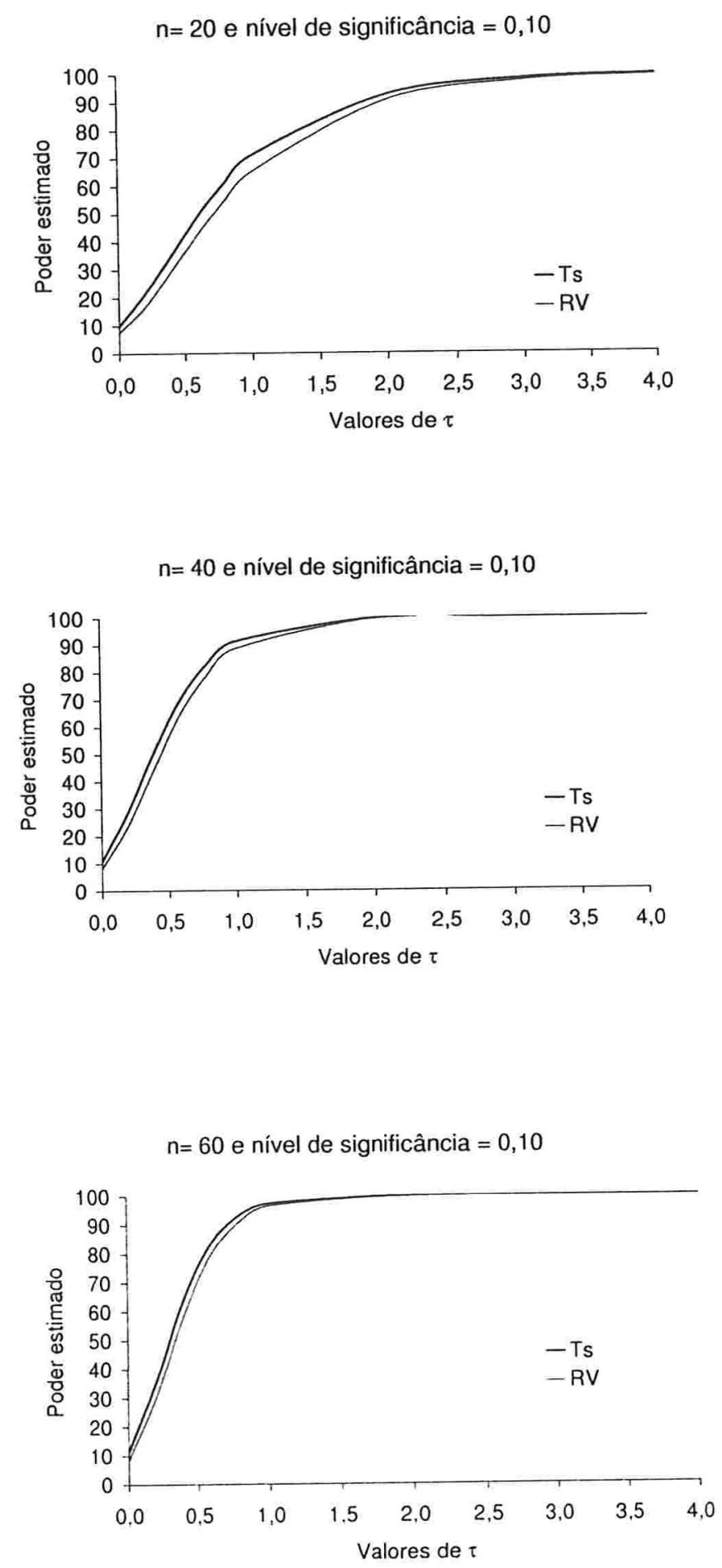
Figura 5.2: Gráficos do poder estimado das estatísticas $T_{S}$ e $R V$ para $\mathrm{n}=20,40$ ou 60 e nível de significância de $5 \%$ para dados gerados a partir de uma distribuição t-Student multivariada com 5 graus de liberdade.

$\mathrm{n}=20$ e nivel de significância $=0,05$

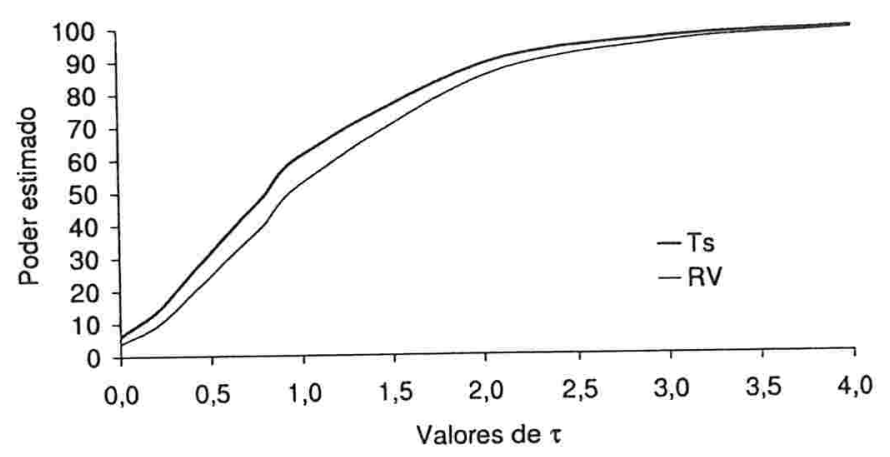

$\mathrm{n}=40$ e nível de significância $=0,05$

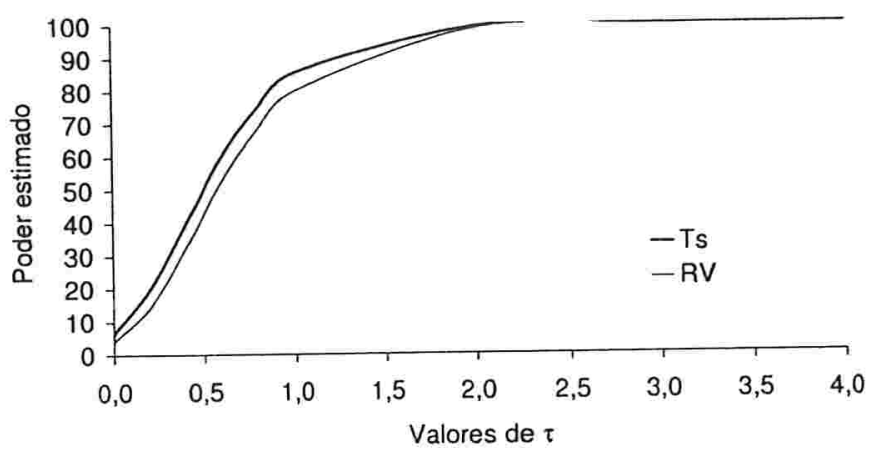

$n=60$ e nível de significância $=0,05$

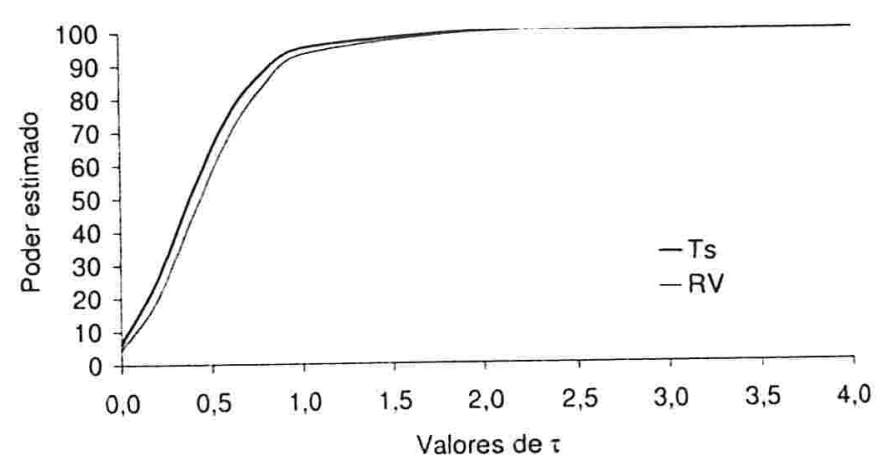


Figura 5.3: Gráficos do poder estimado das estatísticas $T_{S}$ e $R V$ para $n=20,40$ ou 60 e nível de significância de $1 \%$ para dados gerados a partir de uma distribuição t-Student multivariada com 5 graus de liberdade.

$n=20$ e nível de significância $=0,01$

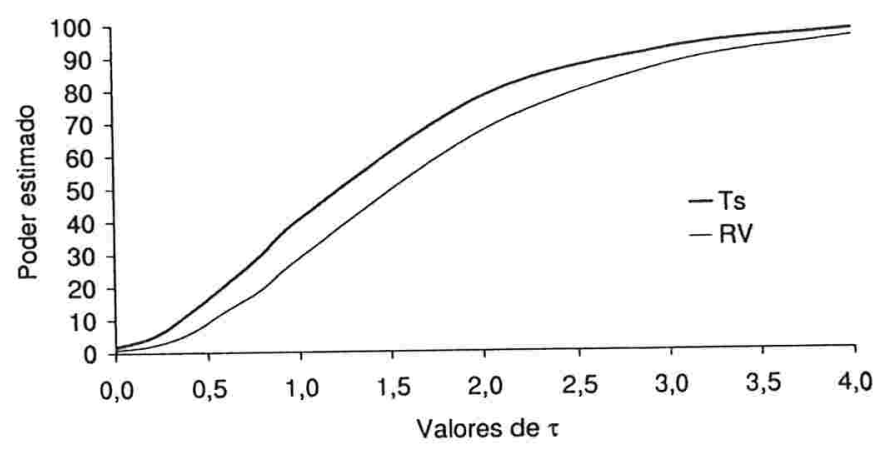

$\mathrm{n}=40$ e nível de significância $=0,01$

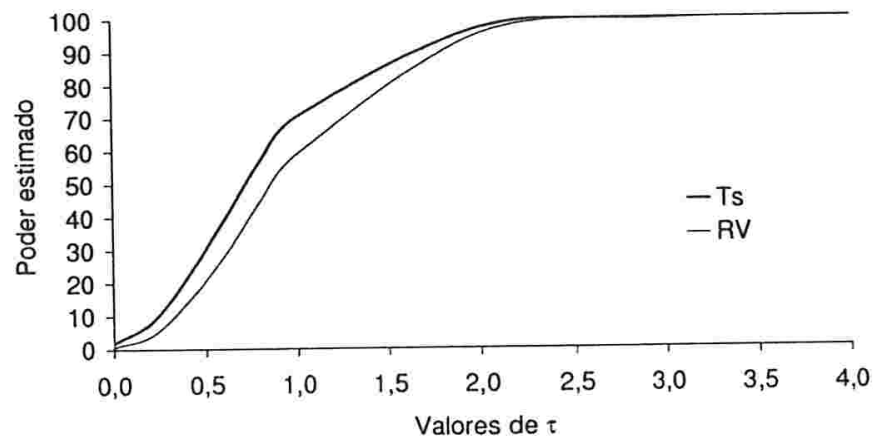

$\mathrm{n}=60$ e nível de significància $=0,01$

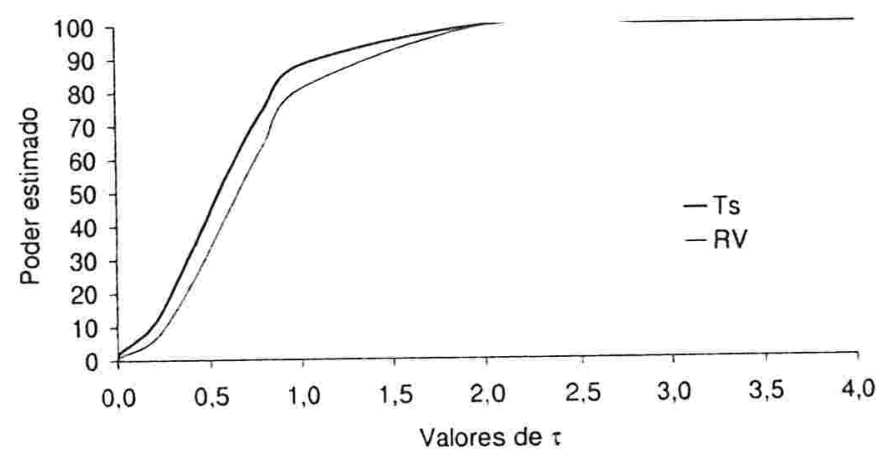


Tabela 5.2: Valores estimados do poder para o teste de um componente de variância para dados gerados a partir de uma distribuição t-Student multivariada com 10 graus de liberdade.

\begin{tabular}{|c|c|c|c|c|c|c|c|c|c|c|c|}
\hline \multirow[b]{2}{*}{$n$} & \multirow[b]{2}{*}{$\alpha$} & \multirow[b]{2}{*}{ Estatística } & \multicolumn{9}{|c|}{$\bar{\tau}$} \\
\hline & & & 0,0 & 0,2 & 0,4 & 0,6 & 0,8 & 1,0 & 2,0 & 3,0 & 4,0 \\
\hline \multirow[t]{6}{*}{20} & $10 \%$ & $\overline{T_{S}}$ & 10,8 & 23,0 & 38,0 & 52,8 & 264,7 & 72,8 & 94,5 & $\overline{99,0}$ & 99,6 \\
\hline & & RV & 7,6 & 17,3 & 30,2 & 44,9 & 56,4 & 66,4 & 92,3 & 98,6 & 99,3 \\
\hline & $5 \%$ & $T_{S}$ & 6,5 & 15,7 & 27,7 & 42,4 & 53,8 & 64,1 & 91,4 & 98,3 & 99,1 \\
\hline & & RV & 3,4 & 10,0 & 19,6 & 32,9 & 43,2 & 54,6 & 87,1 & 96,9 & 98,5 \\
\hline & $1 \%$ & $T_{S}$ & 2,0 & 6,4 & 13,1 & 25,2 & 34,5 & 46,2 & 82,4 & 94,9 & 97,9 \\
\hline & & RV & 0,5 & 2,5 & 6,5 & 13,9 & 21,3 & 31,7 & 71,2 & 89,6 & 95,8 \\
\hline \multirow[t]{6}{*}{$\overline{40}$} & $10 \%$ & $\overline{T_{S}}$ & 11,4 & 31,9 & 55,3 & 74,1 & 86,9 & $\overline{92,8}$ & $\overline{999,7}$ & 100,0 & 100 \\
\hline & & $\mathrm{RV}$ & 8,0 & 25,1 & 47,4 & 67,6 & 81,7 & 89,7 & 99,4 & 100,0 & 100,0 \\
\hline & $5 \%$ & $\overline{T_{S}}$ & 6,5 & 22,7 & 44,0 & 64,4 & 79,6 & 88,1 & 99,4 & 100,0 & 100,0 \\
\hline & & $\mathrm{RV}$ & 3,9 & 16,0 & 34,0 & 55,4 & 71,3 & 82,7 & 99,2 & 99,9 & 100,0 \\
\hline & $1 \%$ & $T_{S}$ & 2,2 & 10,2 & 25,6 & 44,9 & 62,5 & 75,5 & 98,2 & 99,8 & 100,0 \\
\hline & & $\mathrm{RV}$ & 0,7 & 4,6 & 14,9 & 30,2 & 48,3 & 63,4 & 96,3 & 99,7 & 100,0 \\
\hline \multirow[t]{6}{*}{60} & $10 \%$ & $\overline{T_{S}}$ & 11,1 & 38,1 & 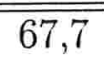 & 86,0 & 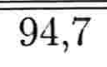 & 98,0 & 100,0 & 10100,0 & 100,0 \\
\hline & & $\mathrm{RV}$ & 7,8 & 31,9 & 60,8 & 82,1 & 92,7 & 96,9 & 100,0 & 100,0 & 100,0 \\
\hline & $5 \%$ & $T_{S}$ & 6,4 & 28,5 & 57,3 & 79,2 & 91,6 & 96,1 & 100,0 & 100,0 & 100,0 \\
\hline & & $\mathrm{RV}$ & 3,5 & 20,5 & 47,2 & 71,1 & 86,8 & 93,7 & 100,0 & 100,0 & 100,0 \\
\hline & $1 \%$ & $T_{S}$ & 2,0 & 13.3 & 35,5 & 61,5 & 80,4 & 90,4 & 99,9 & 100,0 & 100,0 \\
\hline & & RV & 0.6 & 6,9 & 22,8 & 46,7 & 68.3 & 83,0 & 99,6 & 100.0 & 100.0 \\
\hline
\end{tabular}


Figura 5.4: Gráficos do poder estimado das estatísticas $T_{S}$ e $R V$ para $n=20,40$ ou 60 e nível de significância de $10 \%$ para dados gerados a partir de uma distribuição t-Student multivariada com 10 graus de liberdade.
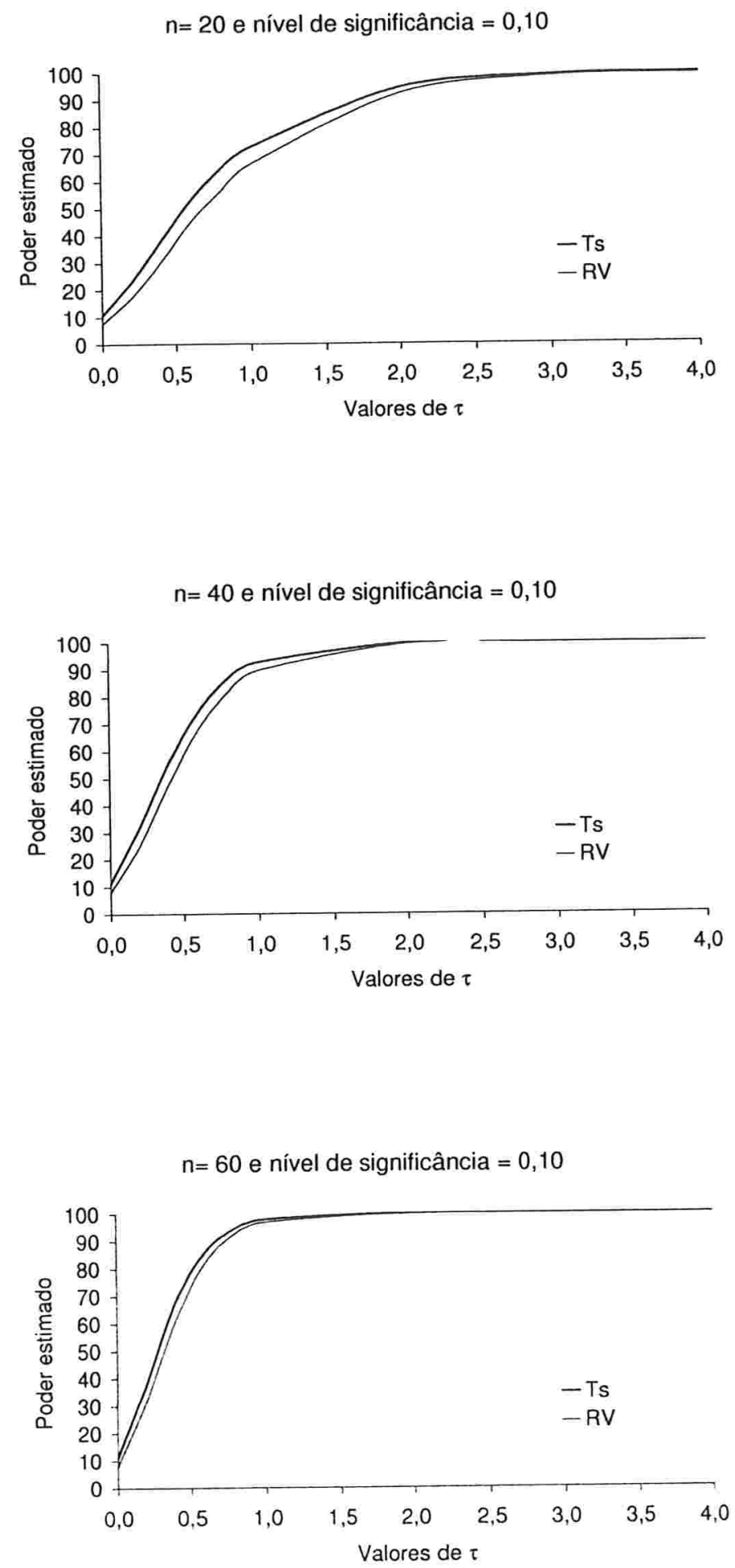
Figura 5.5: Gráficos do poder estimado das estatísticas $T_{S}$ e $R V$ para $\mathrm{n}=20,40$ ou 60 e nível de significância de $5 \%$ para dados gerados a partir de uma distribuição t-Student multivariada com 10 graus de liberdade.

$\mathrm{n}=20$ e nivel de significância $=0,05$

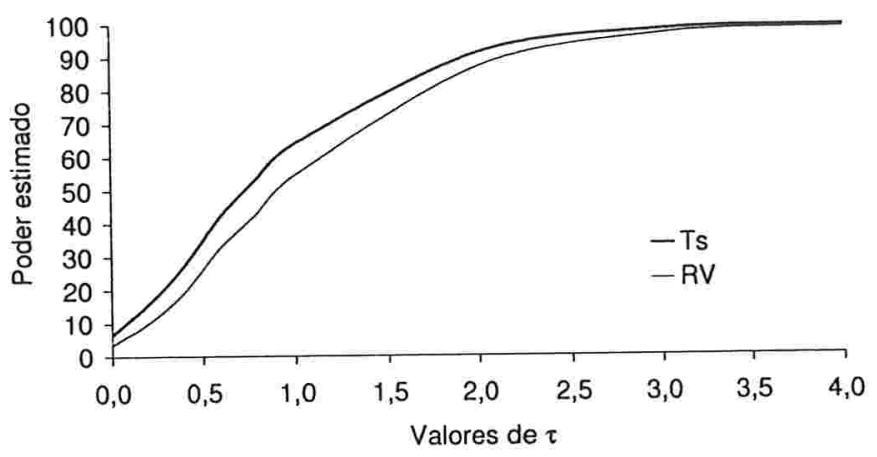

$\mathrm{n}=40$ e nível de significância $=0,05$



$\mathrm{n}=60$ e nível de significância $=0,05$

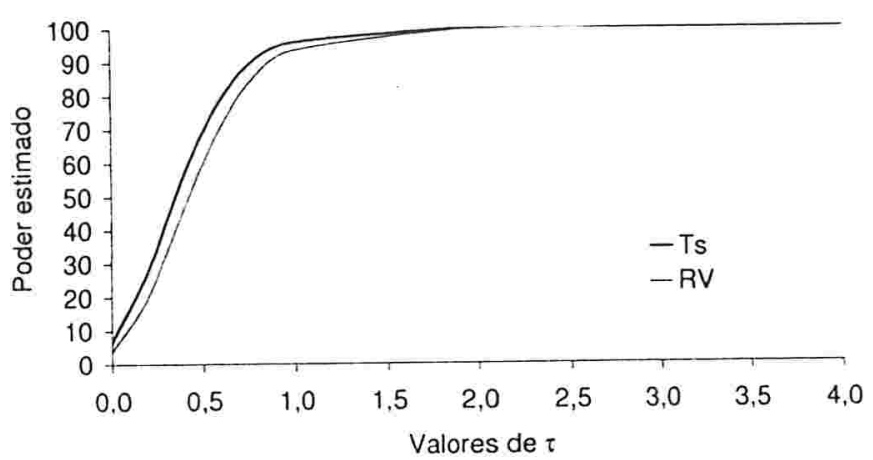


Figura 5.6: Gráficos do poder estimado das estatísticas $T_{S}$ e $R V$ para $n=20,40$ ou 60 e nível de significância de $1 \%$ para dados gerados a partir de uma distribuição t-Student multivariada com 10 graus de liberdade.

$\mathrm{n}=20$ e nível de significância $=0,01$

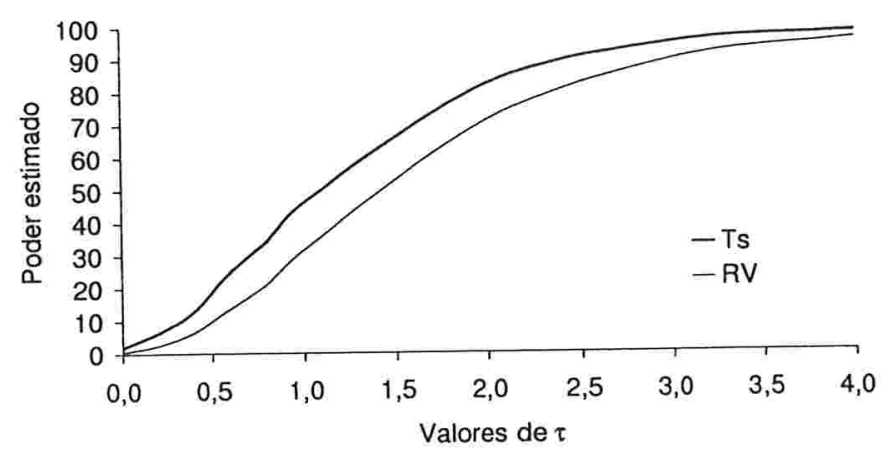

$\mathrm{n}=40$ e nível de significância $=0,01$

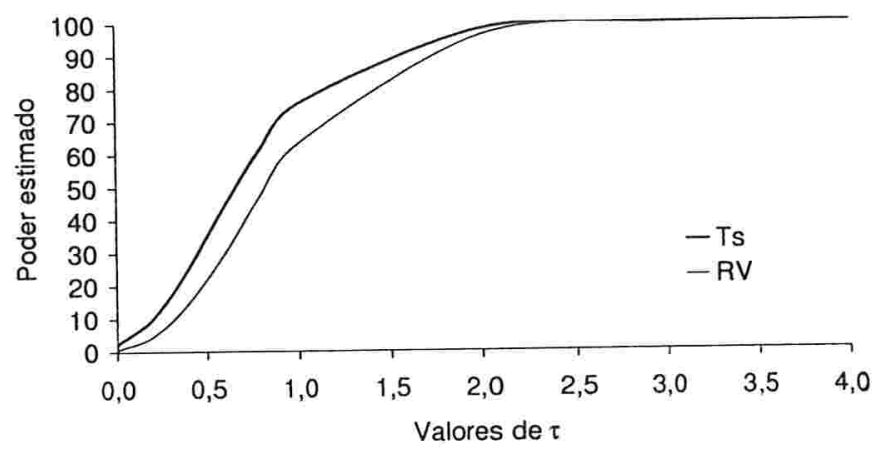

$n=60$ e nivel de significância $=0,01$




Tabela 5.3: Valores estimados do poder para o teste de um componente de variância para dados gerados a partir de uma distribuição exponencial potência multivariada com $K=0,5$.

\begin{tabular}{|c|c|c|c|c|c|c|c|c|c|c|c|}
\hline & & & & & & & $\tau$ & & & & \\
\hline $\mathrm{n}$ & $\alpha$ & Estatística & 0,0 & 0,2 & 0,4 & 0,6 & 0,8 & 1,0 & 2,0 & 3,0 & 4,0 \\
\hline \multirow[t]{6}{*}{20} & $10 \%$ & $\overline{T_{S}}$ & 11,0 & 22,4 & 32,7 & 44,2 & 54,0 & 62,5 & 83,5 & 91,6 & 94,0 \\
\hline & & $\mathrm{RV}$ & 11,0 & 22,4 & 32,7 & 44,3 & 54,0 & 62,5 & 83,3 & 91,6 & 93,9 \\
\hline & $5 \%$ & $T_{S}$ & 7,0 & 15,4 & 24,1 & 34,5 & 43,8 & 51,5 & 76,5 & 86,4 & 91,0 \\
\hline & & $\mathrm{RV}$ & 7,0 & 14,9 & 23,7 & 33,9 & 43,0 & 50,9 & 75,9 & 85,9 & 90,6 \\
\hline & $1 \%$ & $T_{S}$ & 2,6 & 7,0 & 12,0 & 19,1 & 26,6 & 33,8 & 60,5 & 73,3 & 80,7 \\
\hline & & $\mathrm{RV}$ & 2,4 & 6,3 & 11,0 & 17,5 & 24,7 & 32,2 & 58,2 & 71,4 & 78,7 \\
\hline \multirow[t]{6}{*}{40} & $10 \%$ & $\overline{T_{S}}$ & 12,2 & 28,4 & 47,0 & 61,6 & 74,5 & 83,2 & 97,0 & 99,5 & 99,8 \\
\hline & & $R V$ & 12,6 & 29,0 & 47,7 & 62,3 & 75,1 & 83,6 & 97,2 & 99,5 & 99,8 \\
\hline & $5 \%$ & $T_{S}$ & 7,4 & 20,0 & 36,1 & 50,9 & 65,1 & 75,3 & 95,2 & 98,8 & 99,5 \\
\hline & & RV & 7,5 & 20,2 & 36,7 & 51,0 & 65,3 & 75,8 & 95,2 & 98,9 & 99,5 \\
\hline & $1 \%$ & $T_{S}$ & 2,5 & 8,7 & 19,6 & 33,2 & 46,0 & 57,8 & 88,3 & 95,4 & 97,8 \\
\hline & & $\mathrm{RV}$ & 2,4 & 8,6 & 19,2 & 32,8 & 45,5 & 57,1 & 87,8 & 95,2 & 97,6 \\
\hline \multirow[t]{6}{*}{60} & $10 \%$ & $\overline{T_{S}}$ & 12,1 & 33,2 & 55,7 & 74,9 & 86,0 & 93,2 & "99,6 & 100,0 & 100,0 \\
\hline & & $\mathrm{RV}$ & 12,7 & 34,2 & 57,0 & 75,6 & 86,6 & 93,5 & 99,6 & 100,0 & 100,0 \\
\hline & $5 \%$ & $T_{S}$ & 7,7 & 23,5 & 44,3 & 65,6 & 79,6 & 88,5 & 99,2 & 99,9 & 100,0 \\
\hline & & $\mathrm{RV}$ & 8,0 & 24,3 & 44,9 & 66,0 & 79,9 & 88,8 & 99,2 & 99,9 & 100,0 \\
\hline & $1 \%$ & $T_{S}$ & 2,5 & 11,0 & 26,6 & 46,1 & 62,8 & 75,8 & 96.8 & 99,4 & 99,9 \\
\hline & & RV & 2,6 & 11,1 & 26,8 & 46,2 & 62.9 & 75,5 & 96,8 & 99.5 & 99,8 \\
\hline
\end{tabular}


Figura 5.7: Gráficos do poder estimado das estatísticas $T_{S}$ e $R V$ para $n=20,40$ ou 60 e nível de significância de $10 \%$ para dados gerados a partir de uma distribuição exponencial potência multivariada com $K=0,5$.
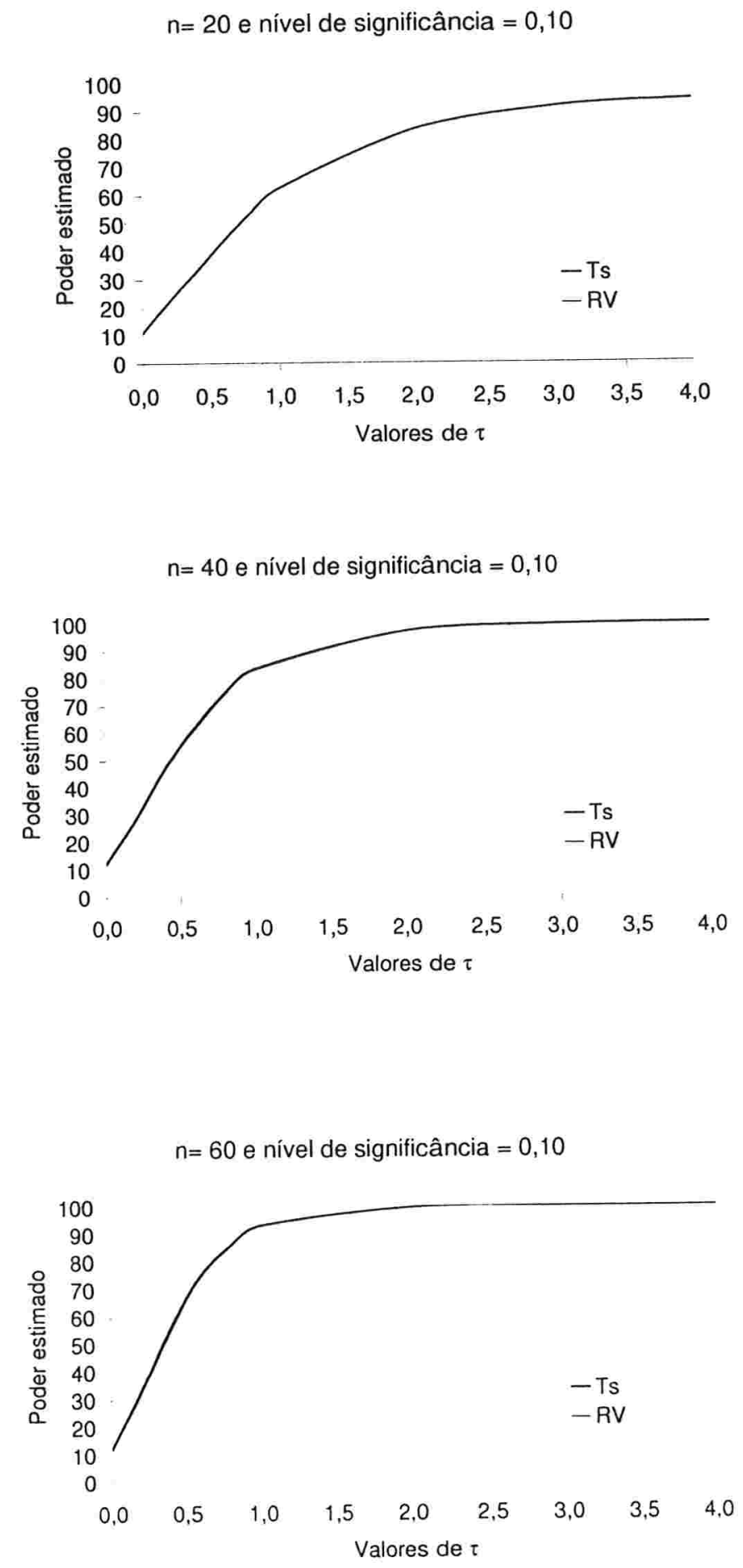
Figura 5.8: Gráficos do poder estimado das estatísticas $T_{S}$ e $R V$ para $\mathrm{n}=20,40 \mathrm{ou}$ 60 e nível de significância de $5 \%$ para dados gerados a partir de uma distribuição exponencial potência multivariada com $K=0,5$.

$\mathrm{n}=20$ e nível de significância $=0,05$

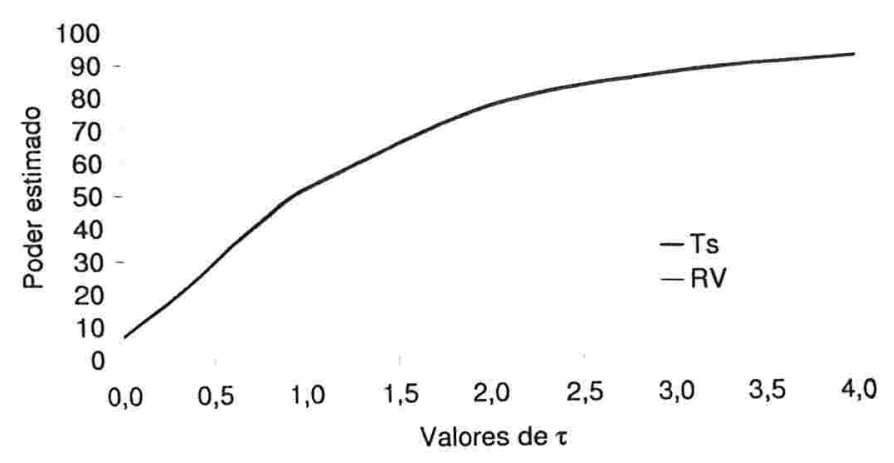

$n=40$ e nivel de significância $=0,05$

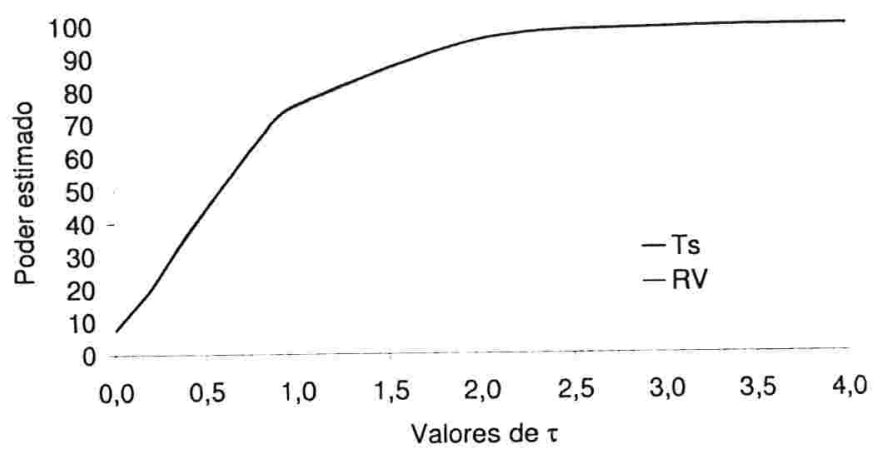

$\mathrm{n}=60$ e nível de significância $=0,05$

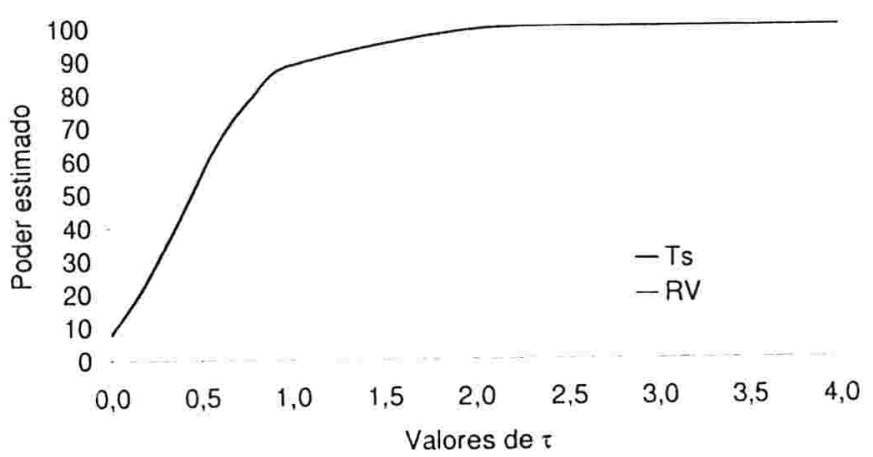


Figura 5.9: Gráficos do poder estimado das estatísticas $T_{S}$ e $R V$ para $n=20,40$ ou 60 e nível de significância de $1 \%$ para dados gerados a partir de uma distribuição exponencial potência multivariada com $K=0,5$.

$$
\mathrm{n}=20 \text { e nível de significância }=0,01
$$

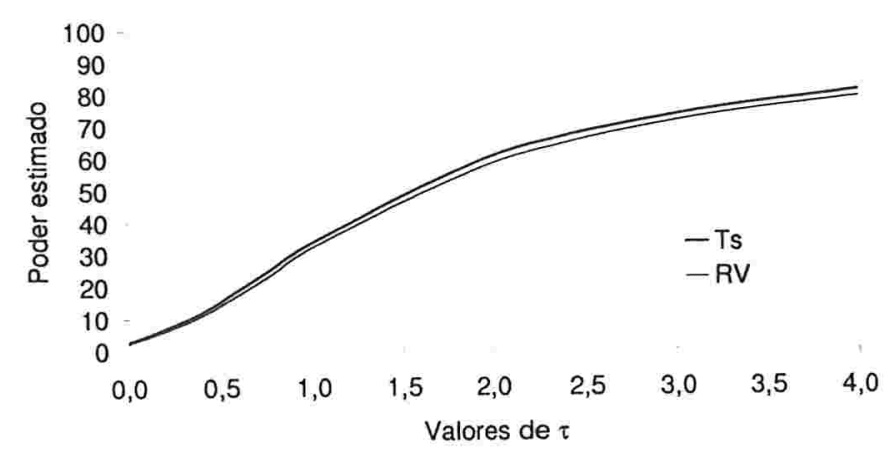

$\mathrm{n}=40$ e nível de significância $=0,01$

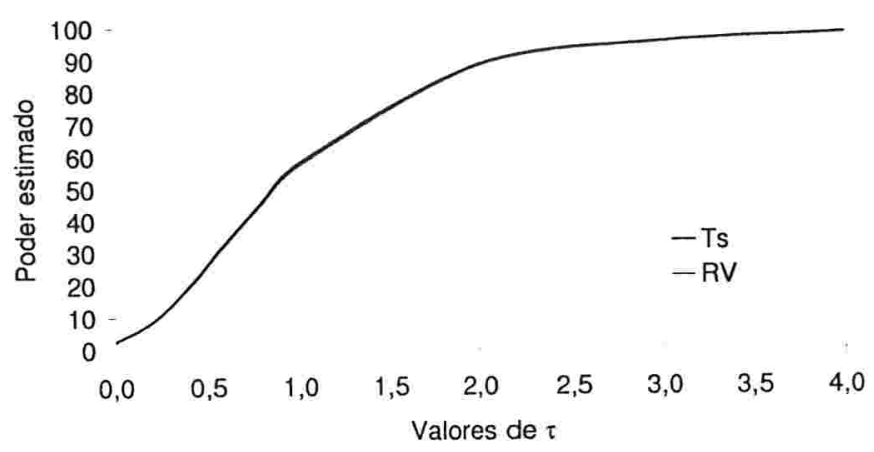

$n=60$ e nivel de significância $=0,01$

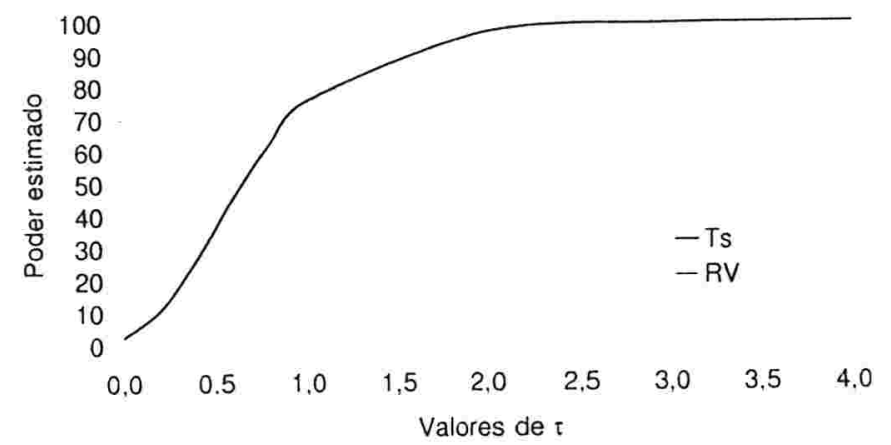




\section{Conclusões}

Neste trabalho generalizamos os modelos de efeitos aleatórios para as distribuições elípticas das quais a normal é um caso particular. Procuramos trabalhar os principais aspectos dos modelos mistos, entre eles a estimação dos efeitos fixos e aleatórios, bem como dos componentes de variância. Também trabalhamos com resíduos e pontos de alavanca para modelos elípticos lineares mistos.

Uma grande ênfase foi dada ao processo de escolha da matriz de variânciascovariâncias que modela a dependência entre as observações feitas dentro de cada "cluster". Em particular, desenvolvemos o teste do tipo escore proposto inicialmente por Silvapulle e Silvapulle (1995) no contexto geral de testes com restrição nos parâmetros. Para o caso elíptico, essa estatística apresenta-se como uma boa alternativa para testar componentes de variância, pois, além de eliminar a influência dos parâmetros de perturbação, também requer que o modelo seja ajustado somente sob a hipótese nula, ou seja, o modelo mais simples. Vale lembrar que os programas estatísticos usuais (SAS, Splus, SPSS etc) ainda não apresentam subrotinas para ajustar modelos elípticos, entretanto, nós desenvolvemos programas na linguagem Ox para ajustar os modelos elípticos lineares mistos e testar hipóteses acerca dos componentes de variância.

Nas aplicações, podemos observar a superioridade de outras distribuições elípticas com relação à distribuição normal no caso em que existem observações aberrantes presentes nos dados. As estimativas dos erros-padrão foram, em geral, menores, produzindo, portanto, estimativas mais precisas. Adicionalmente, ao acomodar essas observações aberrantes obtivemos testes para os componentes de variância mais robustos. Em algumas aplicações podemos ver inclusive mudança 
de decisão nos testes para os componentes de variância sob distribuição normal e outra elíptica com caudas mais pesadas, indicando que a suposição de uma distribuição mais adequada aos dados com observações aberrantes pode proporcionar a escolha de uma estrutura de dependência também mais adequada.

É importante observar que a metodologia desenvolvida neste trabalho vale para as distribuições que satisfazem as condições de regularidade, incluindo também algumas com caudas mais leves do que as da normal como, por exemplo, a distribuição exponencial potência com $-1<K<0$ ou a logística-I. Todavia, dado o enfoque específico deste trabalho em lidar com observações aberrantes, não apresentamos exemplos para essa situação, em que pesos menores são atribuídos aos pontos com menores resíduos, diferentemente dos modelos sob suposição de caudas mais pesadas do que as da normal.

Por fim, podemos ver pelo estudo de simulação que a estatística do tipo escore tende a ser tão poderosa quanto a estatística da razão de verossimilhanças quando assumimos distribuições t-Student com 5 e 10 graus de liberdade e exponencial potência com $K=0,5$. Ressaltamos novamente que, para estender esses resultados para outras distribuições elípticas, ou para teste com mais efeitos aleatórios envolvidos, outros estudos de simulação devem ser propostos.

Como trabalhos futuros propomos :

- A extensão dos estudos de simulação apresentados no Capítulo 5 para outras distribuições e hipóteses mais complexas;

- A extensão do teste do tipo escore para testar componentes de variância no modelo (2.1.2), ou seja, no caso em que os componentes de variância estão na fronteira do espaço paramétrico, como feito no trabalho de Giampaoli (1999) para o caso normal;

- Estudos mais detalhados das estimativas de máxima verossimilhança para os paràmetros $\gamma=\left(\sigma^{2}, \tau^{T}\right)^{T}$ através do processo iterativo (2.3.1), bem como as condições de regularidade de $\widehat{\boldsymbol{\theta}}$; 
- Desenvolvimento do método de estimação de máxima verossimilhança restrita para os modelos elípticos lineares mistos, de forma a obter estimativas menos viesadas para os componentes de variância;

- Aplicações do modelo proposto em outras áreas de conhecimento, como por exemplo, na área de finanças em que é comum a presença de observações extremas;

- Extensão dos modelos elípticos lineares mistos para distribuições assimétricas, trabalho já iniciado em Davila (2004) para o caso simples de um componente de variância no intercepto. O desenvolvimento dos testes do tipo escore também pode ser feito para esses modelos. 


\section{Apêndice A - Probabilidades de Nivel}

\subsection{Caso de $k=3$ restrições}

Para o caso de $k=3$ restrições os pesos ficam dados por (vide, por exemplo, Wolak, 1987)

$$
\begin{aligned}
& \omega_{0}(3 ; \Delta)=\frac{1}{2}-\omega_{2}(3 ; \Delta) ; \\
& \omega_{1}(3 ; \Delta)=\frac{1}{2}-\omega_{3}(3 ; \Delta) ; \\
& \omega_{2}(3 ; \Delta)=\frac{1}{4} \pi^{-1}\left\{3 \pi-\cos ^{-1}\left(\rho_{12.3}\right)-\cos ^{-1}\left(\rho_{13.2}\right)-\cos ^{-1}\left(\rho_{23.1}\right)\right\} \mathrm{e} \\
& \omega_{3}(3 ; \Delta)=\frac{1}{4} \pi^{-1}\left\{2 \pi-\cos ^{-1}\left(\rho_{12}\right)-\cos ^{-1}\left(\rho_{13}\right)-\cos ^{-1}\left(\rho_{23}\right)\right\},
\end{aligned}
$$

em que $\rho_{i j}$ denota o elemento $(i, j)$ da matriz de correlações lineares associadas à matriz $\Delta$, enquanto $\rho_{i j . t}$ 's correspondem aos coeficientes de correlação linear parcial, os quais são definidos por

$$
\rho_{i j . t}=\frac{\rho_{i j}-\rho_{i t} \rho_{j t}}{\sqrt{\left(1-\rho_{i t}^{2}\right)\left(1-\rho_{j t}^{2}\right)}} .
$$




\subsection{Caso de $k=4$ restrições}

No caso de $k=4$ restrições temos as expressões abaixo para os pesos (vide, por exemplo, Wolak 1987)

$$
\begin{aligned}
\omega_{0}(4 ; \Delta)= & \frac{1}{2}-\omega_{4}(4 ; \Delta)-\omega_{2}(4 ; \Delta) \\
\omega_{1}(4 ; \Delta)= & -\frac{1}{2}+\frac{1}{8 \pi} \sum_{i>j ; i, j \neq k} \cos ^{-1}\left(\rho_{i j . k}\right) ; \\
\omega_{2}(4 ; \Delta)= & \frac{1}{4 \pi^{2}} \sum_{i>j, k>\ell ; \ell \neq i, j} \cos ^{-1}\left(\rho_{i j}\right)\left\{\pi-\cos ^{-1}\left(\rho_{k \ell . i j}\right)\right\} \\
\omega_{3}(4 ; \Delta)= & \frac{1}{2}-\omega_{1}(4 ; \Delta) \mathrm{e} \\
\omega_{4}(4 ; \Delta)= & \frac{1}{16}+\frac{1}{8 \pi}\left\{\sin ^{-1}\left(\rho_{12}\right)+\sin ^{-1}\left(\rho_{13}\right)+\sin ^{-1}\left(\rho_{14}\right)\right. \\
& \left.+\sin ^{-1}\left(\rho_{23}\right)+\sin ^{-1}\left(\rho_{24}\right)+\sin ^{-1}\left(\rho_{34}\right)\right\}+\frac{1}{4 \pi^{2}} \eta,
\end{aligned}
$$

em que $\eta$ (vide, Childs, 1967; Sun, 1988) é dado por

$$
\eta=\sum_{k=2}^{4} \frac{\rho_{1 k}}{\sqrt{1-\rho_{1 k} t^{2}}} I_{2}\left(R_{2}^{1, k}\right)
$$

com $I_{2}\left(R_{2}^{1,2}\right), I_{2}\left(R_{2}^{1,3}\right)$ e $I_{2}\left(R_{2}^{1,4}\right)$ sendo integrais no intervalo $(0,1)$ dadas abaixo

$$
\begin{aligned}
& I_{2}\left(R_{2}^{1,2}\right)=\int_{0}^{1} \sin ^{-1}\left(\frac{r_{34}^{12}(t)}{\sqrt{r_{33}^{12}(t) r_{44}^{12}(t)}}\right) ; \\
& I_{2}\left(R_{2}^{1,3}\right)=\int_{0}^{1} \sin ^{-1}\left(\frac{r_{24}^{13}(t)}{\sqrt{r_{22}^{13}(t) r_{44}^{13}(t)}}\right) \mathrm{e} \\
& I_{2}\left(R_{2}^{1,4}\right)=\int_{0}^{1} \sin ^{-1}\left(\frac{r_{23}^{14}(t)}{\sqrt{r_{22}^{14}(t) r_{33}^{14}(t)}}\right),
\end{aligned}
$$

em que

$$
\begin{aligned}
r_{i j}^{1 k}(t)= & \left\{\rho_{i j}-\rho_{k i} \rho_{k j}-t^{2}\left(\rho_{1 k}^{2} \rho_{i j}+\rho_{1 i} \rho_{1 j}\right.\right. \\
& \left.\left.-\rho_{1 k} \rho_{1 i} \rho_{k j}-\rho_{1 k} \rho_{1 j} \rho_{k i}\right)\right\},
\end{aligned}
$$


$i, j, k=1,2,3$. A correlação parcial $\rho_{k \ell . i j}$ é definida abaixo

$$
\rho_{k \ell . i j}=\frac{\rho_{k \ell . i}-\rho_{k j . i} \rho_{\ell j . i}}{\sqrt{\left(1-\rho_{k j . i}^{2}\right)\left(1-\rho_{\ell j . i}^{2}\right)}} .
$$




\section{Referências}

Andreoni, S. (1989). Modelos de Efeitos Aleatórios para Análise de Dados Longitudinais não Balanceados em Relação ao Tempo. Dissertação de mestrado. IME/USP, São Paulo.

Borges L.H.B.; Villas Boas L. S.; Warren-Santoro S.H.; Estevam M.P. Uip D.E., Savalli C.; Curi M. e Pannuti C. S. (2001). Effect of highly active antiretroviral therapy on cytomegalovirus antigenaemia in AIDS patiens. International Journal of STD and AIDS, 12, 234-238.

Childs, D. P. (1967). Reduction of the multivariate normal integral to characteristic form. Biometrika,54, 293-300.

Cysneiros, F. J. A. (2004). Métodos Restritos e Validação de Modelos Simétricos de Regressão. Tese de doutoramento. IME/USP, São Paulo.

Cysneiros, F. J. A. e Paula, G. A. (2004). One-sided tests in linear models with multivariate t-distribution. Communications in Statistics - Simulation and Computation, 33, 747-771.

Davila, V. H. L. (2004). Modelos Lineares Mistos Assimétricos. Tese de Doutorado, IME-USP.

Doornik, J.A. (2001). Ox: an Object-oriented Matrix Programming Language, 4th ed. London: Timberlake Consultants and Oxford: http://www. nuff. ox.ac.uk/Users/Doornik/. 
Fang, K.T.; Kotz, S. e Ng. K.W. (1990). Symmetric Multivariate and Related Distributions. London: Chapman \& Hall.

Fernandez, C. e Steel, M.F.J. (1999). Multivariate student-t regression models: Pitfalls and inference. Biometrika, 86, 153-167.

Galea, M. ; Paula, G. A. e Cysneiros, F. J. A. (2005). On diagnostic in Symmetrical Non-linear Models. Statistics and Probability letters, 73(4), 459-467.

Giampaoli, V. (1999). Inferência Estatística para Modelos Lineares com Restrições nos Parâmetros em Condições Regulares e não Regulares. Tese de doutoramento. IME/USP, São Paulo.

Gómez, E.; Gómez-Villegas, M. A. e Marín, J. M. (1998). A multivariate generalization of the power exponential family of distributions. Communications in Statistics - Theory and Methods, 27, 589-600.

Graybill, F. A. (1983). Matrices with Applications in Statistics. 2ed. California: Wadsworth Publishing Company.

Hilden-Minton, J.A. (1995). Multilevel Diagnostics for Mixed and Hierarchical Linear Models. PhD Thesis. University of California, Los Angeles.

Johnson, R. A. e Wichern, D. W. (1992). Applied Multivariate Statistical Analysis. 3ed. New Jersey: Prentice-Hall, Inc.

Kowalski, J. ; Mendoza-Blanco, J. R. ; Tu, X. M. e Gleser, L. J. (1999). On the difference in inference and prediction between the joint and independent t-error models for seemingly unrelated regressions. Communications in Statistics-Theory and Methods, 28, 2119-2140.

Kudô, A. (1963). A multivariate analogue of the one-sided test. Biometrika, $50,403,418$.

Laird, N. M. e Ware, J. H. (1982). Random effect models for longitudinal data. Biometrics, 38, 963-974. 
Lange, K. L.; Little, J. A. e Taylor, M. G. (1989). Robust statistical modelling using the t distribution. Journal of the American Statistical Association, 84, 881-896.

Lindsey, J. K. (1999). Multivariate elliptically contoured distributions for repeated measurements. Biometrics, 55, 1277-1280.

McCulloch, C. E. e Searle, S. R. (2001). Generalized Linear and Mixed Models. New York: Wiley.

Nobre, J. S. (2003). Métodos de Diagnóstico para Modelos Lineares Mistos. Dissertação de mestrado. IME/USP: São Paulo.

Pan, J. X. e Fang, K. T. (2002). Growth Curve Models and Statistical Diagnostics. New York: Springer.

Paula, G. A. (2004). Modelos de Regressão com Apoio Computacional. São Paulo:IME/USP. http://www. IME. USP. BR/ giapaula.

Paula, G. A. e Artes, R. (2000). One-sided test to assess correlation in linear logistic models using estimating equations. Biometrical Journal, 42, 701714.

Pinheiro, J.C.; Liu, C. e Wu, Y. N. (2001). Efficient algorithms for robust estimation in linear mixed-effect models using the multivariate $t$ distribution. Journal of Computational and Graphical Statistics, 10, 249-276.

Potthoff, R. F. e Roy, S. N. (1964). A generalized multivariate analysis of variance model useful especially for growth curve models. Biometrika, 51, 665-680.

Savalli, C.; Paula G. A. e Cysneiros F. J. A. (2004). Assessment of variance components in elliptical linear mixed models. Statistical Modelling: Proceedings of the $19^{\text {th }}$ International Workshop on Statistical Modeling, Biggeri, 
A., Dreassi, E., Lagazio, C. and Marchi, M. (Eds.). Firenze: Firenze University Press, pp. 144 - 148. Florence, Italy, 144-148.

Savalli, C.; Paula, G. A. e Cysneiros, F. J. A. (2006). Assessment of variance components in elliptical linear mixed models. Statistical Modelling, 6.

Self, S. G. e Liang, K. Y. (1987). Asymptotic properties of maximum likelihood estimators and likelihood ratio tests under nonstandard conditions. Journal of the American Statistical Association, 82, 605-610.

Shapiro, A. (1985). Asymptotic distribution of test statistics in the analysis of moment structures under inequality constraints. Biometrika, 72, 133-144.

Silvapulle, M. J. e Silvapulle, P. (1995). A score test against one-sided alternatives. Journal of the American Statistical Association, 90, 342-349.

St. Laurent, R.T. e Cook, R.D. (1992) . Leverage and superleverage in nonlinear regression. Journal of the American Statistical Association, 87, 985990.

Stram, D.O. e Lee, J.W. (1994) . Variance components testing in the longitudinal mixed effects models. Biometrics, 50, 1171-1177.

Sun, H. J. (1988). A general reduction method for n-variate normal orthant probabilities. Communications in Statistics-Theory and Methods, 17, 39133921.

Taylor, J. e Verbyla, A. (2004). Joint modelling of location and scale parameters of the $\mathrm{t}$ distribution. Statistical Modelling, 4, 91-112.

Vaida, F. e Blanchard, S. (2004). Conditional Akaike Information for Mixed Effects Models. Statistical Modelling: Proceedings of the $19^{\text {th }}$ International Workshop on Statistical Modeling, Biggeri, A., Dreassi, E., Lagazio, C. and Marchi, M. (Eds.). Firenze: Firenze Lniversity Press, pp. $144-148$. Florence, Italy, 101-105. 
Valle, R. B. A. (1994). Distribuições Elípticas: Propriedades, Inferência e Aplicações a Modelos de Regressão. Tese de doutoramento. IME/USP, São Paulo.

Verbeke, G. (1995). The Linear Mixed Model. A Critical Investigation in the Context of Longitudinal Data Analysis. Ph.D thesis, Catholic University of Leuven, Faculty of Science, Department os Mathematics.

Verbeke, G. e Molenberghs, G. (2001). Linear Mixed Models for Longitudinal Data. New York: Springer-Verlag.

Verbeke, G. e Molenberghs, G. (2003). The use of score test for inference on variance components. Biometrics, 59, 254-262.

Vu, H. T. V. e Zhou, S. (1997). Generalization of likelihood ratio tests under nonstandard conditions. Annals of Statistics, 25, 897-916.

Welsh, A. H. e Richardson, A. M. (1997). Approaches of the Robust Estimation of Mixed Models, in Handbook of Statistics (Vol. 15), eds. G. S. Maddala and C. R. Rao, Amsterdam:Elsevier Science, pp. 343-384.

Wei, B.C.; Hu, Y.Q. e Fung, W.K. (1998). Gereralized leverage and its applications. Scandinavian Journal of Statistics, 25, 25-37.

Wolak, F. A. (1987). An exact method for multiple inequality and equality constraints in the linear regression model. Journal of the American Statistical Association, 82, 782-793. 